\title{
Baseline Radionuclide Inventory for the Remote- Handled Low-Level Waste Disposal Facility for Use in the Facility Performance Assessment
}

\author{
A. Jeffrey Sondrup \\ March 2012
}

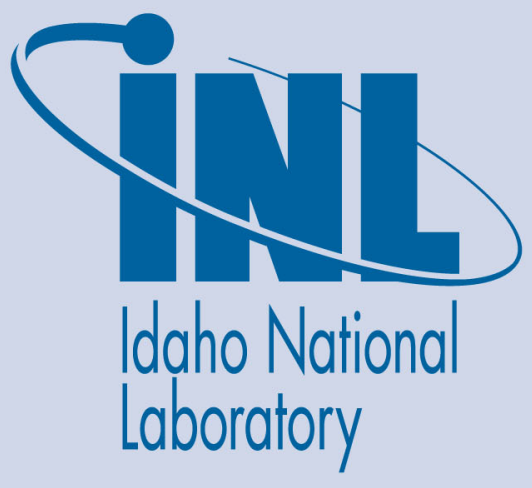

The INL is a U.S. Department of Energy National Laboratory operated by Battelle Energy Alliance 


\title{
Baseline Radionuclide Inventory for the Remote- Handled Low-Level Waste Disposal Facility for Use in the Facility Performance Assessment
}

\author{
A. Jeffrey Sondrup
}

March 2012

Idaho National Laboratory Idaho Falls, Idaho 83415

http://www.inl.gov

Prepared for the

U.S. Department of Energy

Under DOE Idaho Operations Office

Contract DE-AC07-05ID14517 
TEM-10200-1

$03 / 01 / 2012$

ENGINEERING CALCULATIONS AND ANALYSIS

Page 1 of 30

Rev. 06

Title: $\quad$ Facility for Use in the Facility Performance Assessment

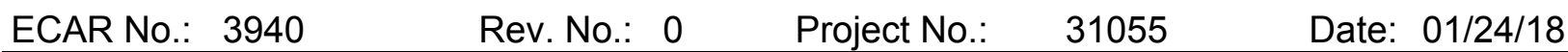

\begin{tabular}{|c|c|c|}
\hline 1. Quality Level (QL) No. & 2 & \multirow{6}{*}{$\begin{array}{l}\text { Professional Engineer's Stamp } \\
\text { NA } \\
\text { (See LWP-10010 for requirements.) }\end{array}$} \\
\hline 2. QL Determination No. & MSA-000202 & \\
\hline 3. Engineering Job (EJ) No. & NA & \\
\hline 4. SSC ID & NA & \\
\hline 5. Building & NA & \\
\hline 6. Site Area & $\begin{array}{l}\text { RH-LLW Disposal } \\
\text { Facility }\end{array}$ & \\
\hline
\end{tabular}

7. Objective/Purpose:

The purpose of this engineering calculations and analysis report (ECAR) is to document the baseline radionuclide inventory anticipated for disposal in the Remote-Handled Low-Level Waste Disposal Facility at Idaho National Laboratory. This inventory will be used in the updated facility performance assessment to obtain an operational disposal authorization statement.

8. If revision, please state the reason and list sections and/or pages being affected:

NA

9. Conclusions/Recommendations:

This document contains the inventory for the first 20 years of operation (i.e., the capacity of the current as-built Remote-Handled Low-Level Waste Disposal Facility) and the inventory for a full 50 years of operation. The inventory is presented by waste generator and waste form and separated into the vault type that will receive the waste. This information is necessary for conducting the dose and exposure assessment for the facility performance assessment. The inventory estimates in this ECAR were taken from other sources. Any adjustments to the inventories from those sources are explained in this ECAR. 
TEM-10200-1

$03 / 01 / 2012$

ENGINEERING CALCULATIONS AND ANALYSIS

Page 2 of 30

Rev. 06

Title: $\quad$ Facility for Use in the Facility Performance Assessment

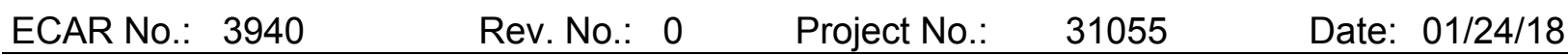

\section{CONTENTS}

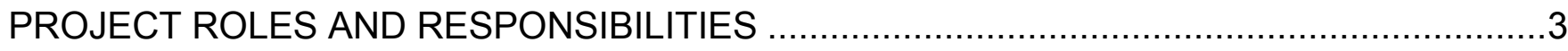

SCOPE AND BRIEF DESCRIPTION

Background: Waste Generators, Waste Forms, and Facility Layout............................ 4

DESIGN OR TECHNICAL PARAMETER INPUT AND SOURCES …................................

RESULTS OF LITERATURE SEARCHES AND OTHER BACKGROUND DATA ….................

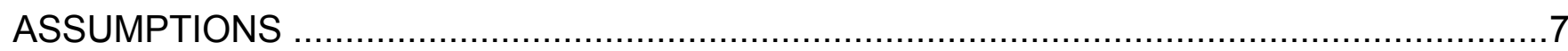

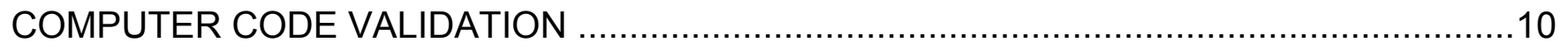

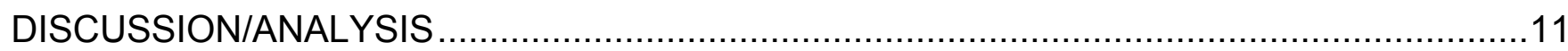

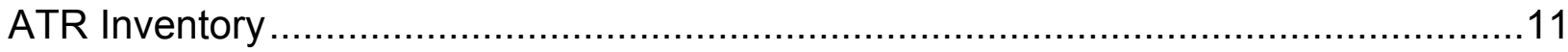

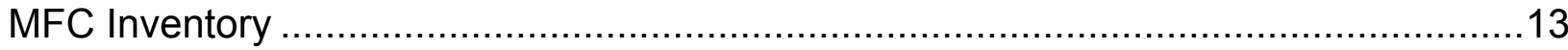

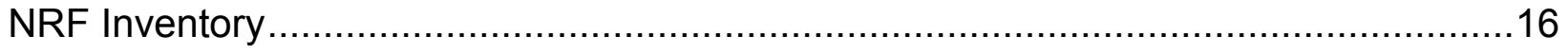

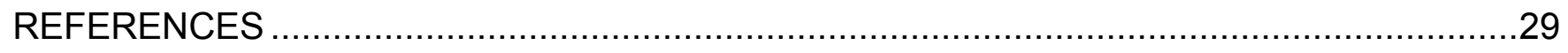

Appendix A - DIF.FOR: A FORTRAN Program for Calculating Radioactive Decay and

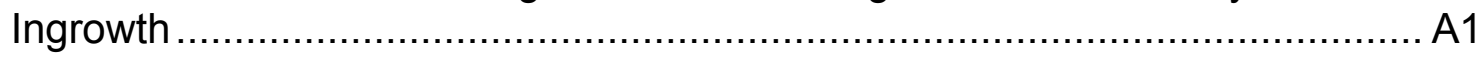

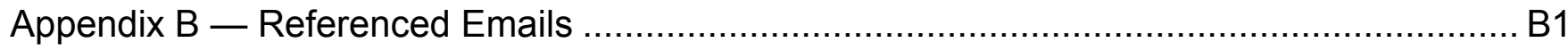


Rev. 06

Baseline Radionuclide Inventory for the Remote-Handled Low-Level Waste Disposal

Title: $\quad$ Facility for Use in the Facility Performance Assessment

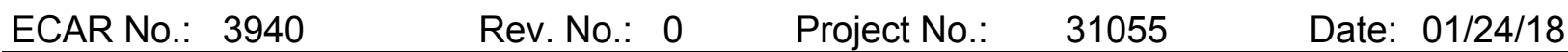

\section{PROJECT ROLES AND RESPONSIBILITIES}

\begin{tabular}{l|l|l|l|}
\multicolumn{1}{c|}{ Project Role } & \multicolumn{1}{|c|}{ Name (Typed) } & Organization & Pages covered (if applicable) \\
\hline Performer & A. Jeffrey Sondrup & BEA \\
Checker $^{a}$ & Annette L. Schafer & BEA \\
Independent Reviewer & K-Spar Inc. \\
CUl Reviewer & Arthur S. Rood & NA \\
Manager & NA & BEA \\
Requestor & Brady J. Orchard & BEA \\
Nuclear Safety & Brett D. Welty & NA \\
Document Owner & NA & Brady J. Orchard & \\
Responsibilities: & & BEA
\end{tabular}
a. Confirmation of completeness, mathematical accuracy, and correctness of data and appropriateness of assumptions.
b. Concurrence of method or approach. See definition in LWP-10106.
c. Concurrence with the document's markings in accordance with LWP-11202.
d. Concurrence of procedure compliance. Concurrence with method/approach and conclusion.
e. Concurrence with the document's assumptions and input information. See definition of acceptance in LWP-10200.

NOTE: $\quad$ Delete or mark "NA" for project roles not engaged. Include ALL personnel and their roles listed above in the eCR system. The list of the roles above is not all inclusive. If needed, the list can be extended or reduced. 
TEM-10200-1

$03 / 01 / 2012$

ENGINEERING CALCULATIONS AND ANALYSIS

Page 4 of 30

Rev. 06

Baseline Radionuclide Inventory for the Remote-Handled Low-Level Waste Disposal

Title: $\quad$ Facility for Use in the Facility Performance Assessment

$\begin{array}{llll}\text { ECAR No.: } 3940 & \text { Rev. No.: } 0 & \text { Project No.: } 31055 \quad \text { Date: } 01 / 24 / 18\end{array}$

\section{SCOPE AND BRIEF DESCRIPTION}

This document contains the baseline radionuclide inventory anticipated for disposal in the Remote-Handled Low-Level Waste (RH-LLW) Disposal Facility at Idaho National Laboratory (INL). This inventory will be used in the updated facility performance assessment (PA) to obtain an operational disposal authorization statement (DAS).

In 2012, the original RH-LLW Disposal Facility PA (DOE-ID 2012) was approved based on a conceptual facility design that would accommodate 50 years of waste disposal in sub-grade concrete vaults. A preliminary DAS was granted to design and construct the facility. Disposal vaults and infrastructure have been constructed to accommodate waste disposal for a period of 20 years. As the 20 -year vault capacity is exhausted, the facility is expected to be expanded to accommodate disposal for an additional 30 years for a total of 50 years of operation. The expansion is expected to incorporate a vault system similar to the existing as-built facility, with changes made to draw on lessons learned during the first 20 years of operations.

This document contains the estimated radiologic waste inventory for the first 20 years of operation (i.e., the capacity of the current as-built facility) and the full 50 years of operation. The inventory is also presented by waste generator and waste form and separated into the vault type that will receive the waste. This information is necessary for conducting the dose and exposure assessment for the PA. All changes to the estimated inventory used in the original PA are explained in this document.

\section{Background: Waste Generators, Waste Forms, and Facility Layout}

The disposal facility will accept RH-LLW generated primarily by operations at the INL Advanced Test Reactor (ATR) Complex, Naval Reactors Facility (NRF), and the Materials and Fuels Complex (MFC). MFC waste consists of legacy waste stored at the Radioactive Scrap and Waste Facility (RSWF) and waste generated from future INL missions.

Waste forms destined for the RH-LLW Disposal Facility include activated metals, ion-exchange resins, and surface-contaminated waste and debris. Activated metals are typically reactor core components replaced during core internal change outs and are made of corrosion-resistant metals such as stainless steel, Inconel (i.e., nickel-chromium-based alloys), Zircaloy (i.e., high-zirconium alloys), or aluminum. The metals contain neutron-activated products present in the metal, with small amounts of activation and fission products on the surface. The ion-exchange resins are used to purify reactor cooling water as part of routine operations at NRF and the ATR Complex. At the ATR Complex, NRF, and MFC, a relatively small amount of miscellaneous surface-contaminated debris is generated and will be included in the waste stream. The debris is mostly residual waste and consists of cuttings and grindings, polishing discs, tools, cable, wiring, glassware, rags, teri-towels, plastic bags, and gaskets.

The current as-built RH-LLW Disposal Facility layout includes five vault types in four vault arrays to accommodate different waste canister sizes (see Figure 1). The Hot Fuel Examination Facility (HFEF)/large concept container (LCC) array has HFEF vaults to accommodate smaller HFEF canisters from MFC and the ATR Complex and LCC vaults built for LCC canisters from NRF. Vaults within each of the other arrays are uniform in size and configuration. 
TEM-10200-1

$03 / 01 / 2012$

Rev. 06

Baseline Radionuclide Inventory for the Remote-Handled Low-Level Waste Disposal

Title: $\quad$ Facility for Use in the Facility Performance Assessment

$\begin{array}{lllll}\text { ECAR No.: } 3940 & \text { Rev. No.: } 0 & \text { Project No.: } & 31055 & \text { Date: } 01 / 24 / 18\end{array}$
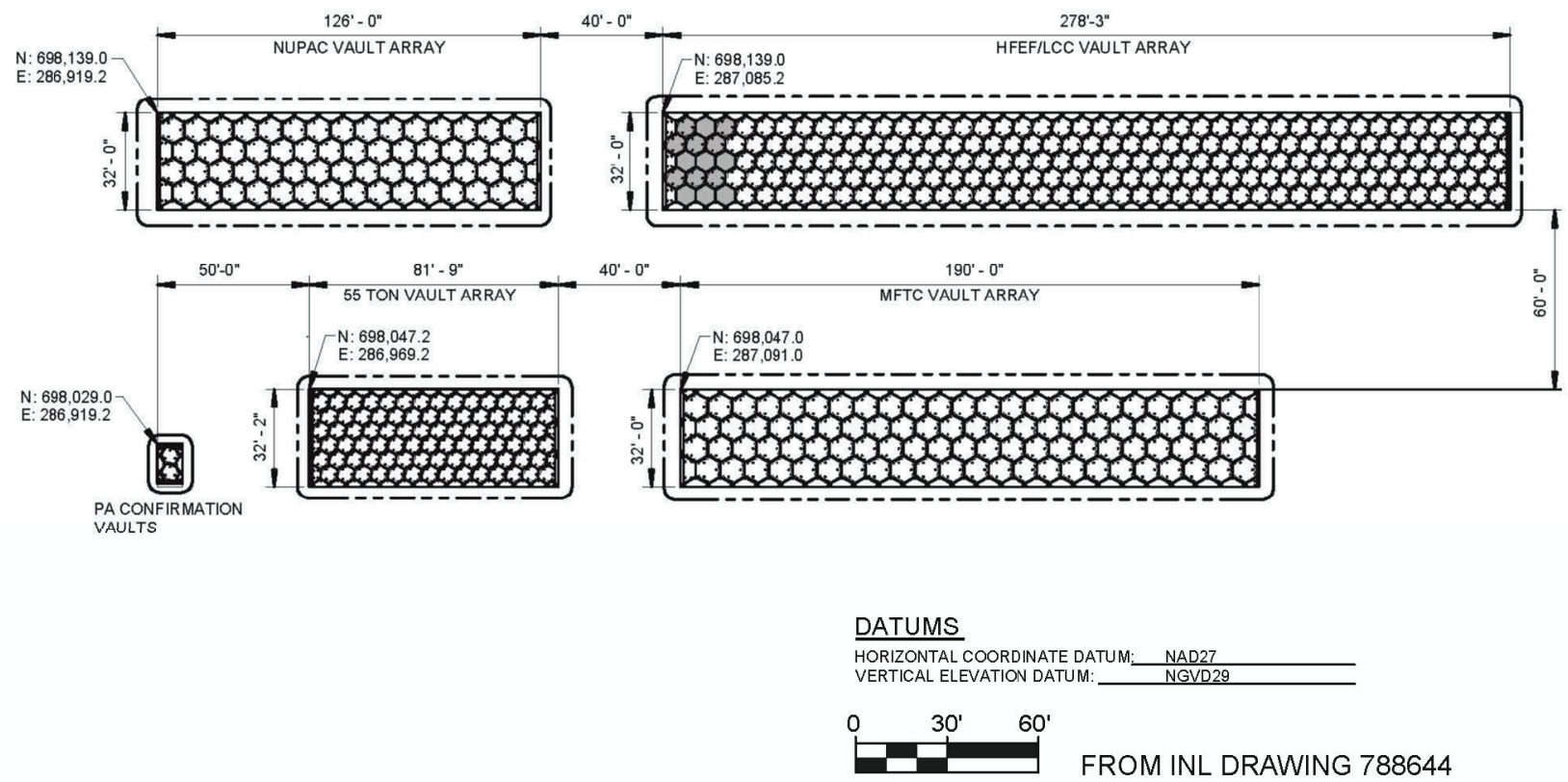

Figure 1. RH-LLW vault yard showing layout of vault arrays.

The five vault types and the waste/canisters they will accommodate are described as follows:

- 55-ton vaults: Vaults in this array were sized to receive activated metals and resins from NRF and to use the 55-ton scrap cask during disposal operations. Vaults of this type are anticipated to be filled during the first 20 years of operation.

- $\quad$ LCC vaults: Vaults in this array were sized to receive activated metal waste and resins from NRF as they transition from the smaller 55-ton waste canisters to the larger LCC canisters.

- HFEF vaults: The bottom/top-loading HFEF-5 cask design (or similar) was identified to be the most suitable transport cask for disposal of remote-handled activated metals and debris from the MFC hot cells and from the ATR canal. The HFEF vaults are located on the west end of the HFEF/LCC vault array. The HFEF vaults have inner partitions that can accommodate 12 HFEF cans per vault.

- NuPac vaults. This array was sized and located to receive resins from the ATR Complex that will be contained in NuPac 14-210L canisters.

- Modified facility transfer container (MFTC) vaults: This vault array was sized to allow receipt of legacy waste currently in storage at RSWF. This waste is a combination of activated metals and surface-contaminated debris. Vaults of this type are expected to be filled during the first 20 years of operation. 
Rev. 06

Baseline Radionuclide Inventory for the Remote-Handled Low-Level Waste Disposal

Title: $\quad$ Facility for Use in the Facility Performance Assessment

$\begin{array}{lllll}\text { ECAR No.: } 3940 \quad \text { Rev. No.: } & 0 & \text { Project No.: } 31055 \quad \text { Date: } 01 / 24 / 18\end{array}$

\section{DESIGN OR TECHNICAL PARAMETER INPUT AND SOURCES}

The RH-LLW Disposal Facility inventory comes from the sources identified in Table 1. The ATR and NRF inventories were provided assuming a 50-year period of disposal. The MFC inventory provides a 20-year and subsequent 30-year inventory. Recommendations for dividing the 50-year ATR and NRF inventories into the 20-year and subsequent 30-year inventories were provided by the waste generators consistent with their expected disposal rates as discussed in the Assumptions section. Other modifications to the inventories in Table 1 that were required to support the calculations and analyses in the PA being submitted to obtain the operational DAS are also discussed in the Assumptions section.

Table 1. Source documentation for the RH-LLW 20-year and 50-year inventory estimates.

\begin{tabular}{|c|c|c|}
\hline Data Source & $\begin{array}{c}\text { Waste } \\
\text { Generator }\end{array}$ & Comments \\
\hline $\begin{array}{l}\text { ECAR-851 } \\
\text { Revision } 3 \\
(2011)\end{array}$ & ATR & $\begin{array}{l}\text { Table } 1 \text { of ECAR- } 851 \text { contains an estimated } 50 \text {-year radionuclide inventory of ATR canal } \\
\text { resins with small amounts of hardware and filters. The } 50 \text {-year ATR resin inventory for the } \\
\text { original PA came directly from ECAR- } 851 \text {. }\end{array}$ \\
\hline $\begin{array}{l}\text { ECAR-854 } \\
\text { Revision } 1 \\
(2010)\end{array}$ & ATR & $\begin{array}{l}\text { Table } 4 \text { of ECAR- } 854 \text { contains an estimated } 50 \text {-year activated metal inventory from ATR } \\
\text { operations. The } 50 \text {-year ATR activated metal inventory for the original PA came directly from } \\
\text { ECAR-854. }\end{array}$ \\
\hline $\begin{array}{l}\text { ECAR-3225 } \\
(2017)\end{array}$ & MFC & $\begin{array}{l}\text { Tables 3, 4, and } 5 \text { of ECAR- } 3225 \text { contain a summary of the legacy radionuclide inventory } \\
\text { stored at RSWF at MFC. This inventory will be disposed of during the first } 20 \text { years of } \\
\text { operation. Table } 6 \text { of ECAR-3225 contains the estimated } 20 \text {-year and } 50 \text {-year inventory to be } \\
\text { generated in the future from operations at MFC. ECAR- } 3225 \text { replaces the information in } \\
\text { ECAR-904 (2011), ECAR-967 (2011), and ECAR-1588 (2011), which were used to determine } \\
\text { the } 50 \text {-year inventory for the original PA. TEV-2921 (2017) summarizes the changes in waste } \\
\text { categorization and reported inventory between the information originally presented in } \\
\text { ECAR-904, ECAR-967, and ECAR-1588 and the information presented in ECAR-3225. The } \\
\text { legacy radionuclide inventories in ECAR-3225 are the inventories when the waste was placed } \\
\text { in the container (i.e., not decayed). The legacy radionuclide inventories presented in this } \\
\text { ECAR have been decayed (including ingrowth of significant progeny) from the generation date } \\
\text { to the assumed facility start date. This is explained further in the Assumptions section. }\end{array}$ \\
\hline $\begin{array}{l}\text { NRF } \\
(2011)\end{array}$ & NRF & $\begin{array}{l}\text { NRF (2011) contains an estimated } 50 \text {-year radionuclide inventory for activated metals, surface } \\
\text { contamination on activated metals, and resins to be generated at NRF. The } 50 \text {-year NRF } \\
\text { inventory for the original PA came directly from NRF (2011). }\end{array}$ \\
\hline
\end{tabular}

This ECAR presents the radionuclide inventory by waste generator and container/vault type. It does not list activated metal inventory by metal type. However, Table 2 contains the approximate weight percent of activated metal types by generator. NRF activated metal waste is approximately $98 \%$ Inconel, or Zircaloy, and $2 \%$ stainless steel. NRF is not expected to ship any aluminum. MFC activated metal waste is expected to be nearly all stainless steel, Inconel, or Zircaloy with $1 \%$ or less expected to be aluminum. Activated metal waste from ATR on the other hand has a much higher percentage of aluminum (approximately $54 \%$ by weight) with the balance ( $46 \%$ by weight) comprised of stainless steel or Inconel. Although the activated metal waste from ATR has a high percentage of aluminum, aluminum is estimated to be less than $0.5 \%$ of the total mass of activated metal waste to be disposed of at the RH-LLW Disposal Facility (INL 2011). ECAR-854 contains the information to determine the radionuclide inventory in each metal type. 
TEM-10200-1

$03 / 01 / 2012$

ENGINEERING CALCULATIONS AND ANALYSIS

Page 7 of 30

Rev. 06

Baseline Radionuclide Inventory for the Remote-Handled Low-Level Waste Disposal

Title: $\quad$ Facility for Use in the Facility Performance Assessment

$\begin{array}{lllll}\text { ECAR No.: } 3940 \quad \text { Rev. No.: } 0 & \text { Project No.: } 31055 \quad \text { Date: } 01 / 24 / 18\end{array}$

Table 2. Weight percent activated metal type by waste generator.

\begin{tabular}{cccc}
\hline Activated Metal Type & $\mathrm{ATR}^{\mathrm{a}}$ & $\mathrm{NRF}^{\mathrm{b}}$ & $\mathrm{MFC}^{\mathrm{c}}$ \\
\hline Inconel/Zircaloy & 4 & 98 & $4-5$ \\
Stainless Steel & 42 & 2 & $94-96$ \\
Aluminum & 54 & 0 & $0.1-1$ \\
\hline
\end{tabular}

a. Determined from information in ECAR-854.

b. Jeff Frazier email to Jeff Sondrup, July 28, 2010.

c. Activated metal waste from MFC is predominately stainless steel (ECAR-3340). To allow for flexibility, future generated waste is assumed to contain small amounts of Inconel, Zircaloy or aluminum.

\section{RESULTS OF LITERATURE SEARCHES AND OTHER BACKGROUND DATA}

Background information is presented in the Discussion/Analysis section and included in the references.

\section{ASSUMPTIONS}

Assumptions used to determine, estimate, and/or justify inventories in the reference documents listed in Table 1 are not presented here. This document lists only the assumptions used to modify inventories from those presented in the references in Table 1 (if applicable), split the activities into 20-year and 50 -year inventories, or assign them to a particular vault type.

1. The first 20-years of disposal operations are assumed to start in year 2018 and run through year 2038, with final facility closure expected in year 2068 (assumes future expansion of disposal capacity to accommodate 50 years of waste generation). For simplicity and to account for uncertainty in the exact start of operations and closure of the facility, the start and closure dates have been rounded to years 2020 and 2070, respectively. Because the PA is based on the inventory at the time of facility closure, a few years uncertainty in the start date is not significant.

2. The 50-year inventories of radionuclides in ATR resins and activated metals provided in ECAR-851 and ECAR-854 are appropriate for the 50-year period of operations estimated to commence in 2020. Although both ECARs state the inventories are for the 50-year period 2015 to 2065, that timeframe is listed only as an example. The averages used in the calculations for number of shipments and projected source terms apply regardless of when the first waste shipment is received (Stacy Nottestad email to Brett Welty, September 27, 2017).

3. The 20-year inventory of resins from ATR in ECAR-851 can be determined by multiplying the 50 -year inventory of each nuclide by the ratio 20/50 (i.e., 0.4). ECAR-851 contains a 50-year inventory. The rate of resin generation will remain the same; therefore, it is appropriate to simply ratio them based on 20 years versus 50 years (Stacy Nottestad email to Brett Welty, September 27, 2017). The 20-year ATR resin inventory is assumed to be disposed of in the NuPac vault array.

4. The 20-year inventory of activated metals from ATR corresponds to the undecayed radionuclide inventory from three core internal changeouts (CICs) and the 50-year inventory corresponds to the inventory from six CICs. Table 4 of ECAR-854 contains the inventory for one CIC; CICs are expected to occur every 10 years. Based on information provided by Battelle Energy Alliance Waste Management Program (Stacy Nottestad email to Brett Welty, September 27, 2017), it is estimated that the inventory equivalent of one $\mathrm{CIC}$ is currently in the ATR canal that will be shipped to the facility after commencement of disposal operations; and there will be an additional two $\mathrm{CICs}$ that 
TEM-10200-1

$03 / 01 / 2012$

Rev. 06

Baseline Radionuclide Inventory for the Remote-Handled Low-Level Waste Disposal

Title: $\quad$ Facility for Use in the Facility Performance Assessment

$\begin{array}{lllll}\text { ECAR No.: } 3940 \quad \text { Rev. No.: } 0 & \text { Project No.: } 31055 \quad \text { Date: } 01 / 24 / 18\end{array}$

will be shipped to the RH-LLW Disposal Facility during the first 20 years of operation. An additional three CICs will be shipped to the expanded facility for a total of six CIC equivalents over 50 years. The one CIC equivalent inventory currently in the ATR canal was not corrected for decay. It is estimated this waste could be 15 to 25 years old at the time the facility opens (Stacy Nottestad email to Jeff Sondrup, October 31, 2017). Not decaying this material provides a conservative estimate for the one $\mathrm{CIC}$ equivalent inventory currently in the canal.

5. ATR activated metals are assumed to be disposed of in HFEF vaults. This is not important to determination of the 20 and 50-year inventories, but it is included here because it was either not included in, or is different from information in the original inventory document (ECAR-854). This is important for assigning the vault array where the waste will be placed.

6. The legacy radionuclide inventories at RSWF presented in ECAR-3225 are the inventories when waste was placed in the container (see "generation date" in Appendix A of ECAR-3225). The legacy RSWF radionuclide inventories presented in this ECAR have been decayed (including ingrowth of significant progeny) from the generation date to the year 2020, when it is estimated disposal of legacy waste will commence at the RH-LLW Disposal Facility (see Assumption 1). The ages of the containers at the estimated time of disposal (estimated 4/1/2020) range from 5 to 55 years, with an average age of 45 years. If disposals commence earlier in year 2018, an excess of 2 years will have been accounted for in the decay calculation. However, as stated in Assumption 1, the PA is based on the inventory at the time of facility closure. Therefore, decaying the inventory to any date prior to facility closure is conservative and a few years uncertainty in the start date is not significant.

Table 3 lists parent radionuclides from the RSWF inventory that have significant progeny. Significant progeny in this context are defined as progeny that are not in secular equilibrium with the parent and change appreciably through decay or ingrowth during the timeframe considered. The parent radionuclides in Table 3 are sorted by half-life. When decaying the legacy inventory, ingrowth of significant progeny was only included for parent radionuclides with half-lives less than 6,537 years (i.e., the half-life of Pu-240). These include Pu-241 (half-life $=14.4$ years), Pu-238 (half-life $=87.7$ years), and Am-241 (half-life $=432$ years). Ingrowth was not included for parent radionuclides with significant progeny and parent half-lives 6,537 years or longer. These include Pu-240 (6,537 years), Pu-239 (24,065 years), U-233 (158,500 years), U-234 (244,500 years), Np-237 (2.14E+06 years), U-236 (2.34E+07 years), U-235 (7.04E+08 years), and U-238 (4.47E+09 years) (half-lives in parentheses). Based on a half-life of 6,537 years, less than $0.6 \%$ of the original inventory would have decayed after 55 years (i.e., age of oldest container). Thus, accounting for decay of radionuclides with half-lives 6,537 years or longer would result in an insignificant difference to the inventory of the parent or ingrowth of progeny. The methodology for calculation of decay and ingrowth is described in Appendix A, along with the FORTRAN program listing and input/output files for doing the calculations. Radionuclide half-lives for decay calculations were taken from Federal Guidance Report No. 13 (EPA 1999). Decay chains used in the FORTRAN program were abbreviated to include only longer-lived decay chain members. As stated previously, short-lived progeny are assumed to be in secular equilibrium with their parent.

Because all legacy radionuclides were decayed, some radionuclides will have decayed to very low activity levels prior to disposal. Therefore, radionuclide activities less than 1E-25 Ci after decay correction were assumed to be zero. 
TEM-10200-1

$03 / 01 / 2012$

ENGINEERING CALCULATIONS AND ANALYSIS

Page 9 of 30

Rev. 06

Baseline Radionuclide Inventory for the Remote-Handled Low-Level Waste Disposal

Title: $\quad$ Facility for Use in the Facility Performance Assessment

$\begin{array}{lllll}\text { ECAR No.: } 3940 \quad \text { Rev. No.: } 0 & \text { Project No.: } 31055 \quad \text { Date: } 01 / 24 / 18\end{array}$

Table 3. Radionuclides in the MFC legacy inventory with significant progeny ${ }^{\text {a }}$

\begin{tabular}{lllllll}
\hline Parent & Half-Life (years) & Progeny1 & Progeny2 & Progeny3 & Progeny3 & Progeny4 \\
\hline Pu-241 $^{\text {b }}$ & 14.4 & Am-241 & Np-237 & U-233 & Th-229 & \\
Pu-238 $^{\text {b }}$ & 87.74 & U-234 & Th-230 & Ra-226 & Pb-210 & \\
Am-241 $^{\text {b }}$ & 432.2 & Np-237 & U-233 & Th-229 & & \\
Pu-240 & 6537 & U-236 & Th-232 & Ra-228 & Th-228 & \\
U-233 & 158500 & Th-229 & & & & \\
U-234 & 244500 & Th-230 & Ra-226 & Pb-210 & & \\
Pu-242 & 376300 & U-238 & U-234 & Th-230 & Ra-226 & Pb-210 \\
Np-237 & $2.14 E+6$ & U-233 & Th-229 & & & \\
U-236 & $2.34 E+7$ & Th-232 & Ra-228 & Th-228 & & \\
U-235 & $7.04 E+08$ & Pa-231 & Ac-227 & & & \\
U-238 & $4.47 E+09$ & U-234 & Th-230 & Ra-226 & Pb-210 & \\
\hline
\end{tabular}

a. Significant progeny are defined as progeny not in secular equilibrium with the parent.

b. Only parent radionuclides with a half-life less than 6,537 years (Pu-240) were included.

7. Legacy waste at RSWF in an outermost waste container listed as "24-in. Liner" or "SLSF" in Tables 3, 4, and 5 of ECAR-3225 will be disposed of in the MFTC vault array during the first 20 years of operation. Legacy waste at RSWF in an "HFEF-5 Outer" outermost waste container (as listed in Tables 3, 4, and 5 of ECAR-3225) will be disposed of in one of the HFEF vaults at the west end of the HFEF/LCC vault array during the first 20 years of operation. Future generated waste from MFC (Table 6 of ECAR-3225) will be in an HFEF-5 container and will be disposed of in one of the 15 HFEF vaults at the west end of the HFEF/LCC vault array during the first 20 years of operation.

8. The 50-year radionuclide inventory for NRF activated metals in NRF (2011) is appropriate for the 50 -year period of operations estimated to commence in 2020. NRF (2011) provides an annual inventory for each radionuclide for years 2015 through 2041 and an average annual inventory to be applied for the years after 2041. Although the reference states the inventories are for a 50-year period beginning in 2015, the inventory can be applied to a 2020 disposal start date by shifting the annual inventories by 5 years (Jeff Frazier email to Brett Welty, September 20, 2017). This applies to both the activation products integral to the metal and the fission products and other radionuclides on the surfaces of the metal components (referred to as "crud" in NRF [2011]). The crud is considered surface contamination in the PA.

9. Because the NRF activated metal and "crud" inventories were provided as a total and a percentage of "crud" for each radionuclide, it was necessary to calculate the fraction of the total inventory for each radionuclide that is an activation product. This was done by simply multiplying the total by the fraction that is not "crud."

10. The 50-year radionuclide inventory for NRF resins in NRF (2011) is appropriate for the 50-year period of operations estimated to commence in 2020. Although the reference provides an average annual resin inventory to be applied to the years 2015 through 2065, the inventory can be applied to a 2020 disposal start date by shifting the annual inventories by 5 years (Jeff Frazier email to Brett Welty, September 20, 2017). 
TEM-10200-1

$03 / 01 / 2012$

ENGINEERING CALCULATIONS AND ANALYSIS

Page 10 of 30

Rev. 06

Title: $\quad$ Facility for Use in the Facility Performance Assessment

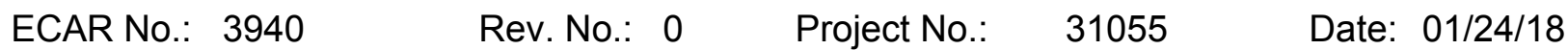

11. The NRF activated metal, "crud," and resin inventories are divided between the 55-ton and HFEF/LCC vault arrays based on the ratio of container volumes used for each year as provided in NRF (2011). For the first 5 years of operation, NRF proposes to ship all waste (i.e., 100\%) in 55-ton containers. During years 6 through 10 as NRF is transitioning to the LCC containers, NRF plans to ship nine to ten 55-ton containers per year (i.e., ten in years 6 and 7 and nine in years 8 through 10) and $11 \mathrm{LCC}$ containers per year. The 55-ton container volume is $2.5 \mathrm{~m}^{3}$ and the LCC container volume is $7.8 \mathrm{~m}^{3}$. Based on the number of containers and these percentages, $23 \%$ of the waste is assumed to be in 55-ton containers in years 6 and 7 and $21 \%$ in years 8 through 10 . The balance of waste during those years is assumed to be in LCC containers. After 10 years, the plan is to ship all waste (i.e., 100\%) in LCC containers. These percentages (shown in the Table 4) are used to apportion the waste for each year.

Table 4. Percentage of NRF waste in 55-ton and LCC containers.

\begin{tabular}{ccc}
\hline Years & $\begin{array}{c}\text { Percent Waste in } \\
\text { 55-ton Containers }\end{array}$ & $\begin{array}{c}\text { Percent Waste in } \\
\text { LCCs }\end{array}$ \\
\hline 1 through 5 & $100 \%$ & $0 \%$ \\
6 and 7 & $23 \%$ & $77 \%$ \\
8 through 10 & $21 \%$ & $79 \%$ \\
11 through 50 & $0 \%$ & $100 \%$ \\
\hline
\end{tabular}

\section{COMPUTER CODE VALIDATION}

All spreadsheet calculations were performed with Microsoft Excel@ $2013(15.0 .4884 .1000) 32$ bit, Part of Microsoft Office Professional Plus 2013 running on a Dell囚 Optiplex 7020 computer (Intel ${ }^{\circledR}$ Core ${ }^{\mathrm{TM}}$ i7-4790 CPU @ $3.60 \mathrm{GHz}$ ) running Microsoft $₫$ Windows $₫ 7$ Enterprise, Service Pack 1. To validate the Excel calculations, the calculation cell formulas were checked for accuracy and a random sample of the calculations were checked by hand. Radionuclide decay with progeny ingrowth was calculated using the FORTRAN program DIF.FOR running on a MAC Pro® Computer with 2 Quad-Core Intel Xeon® Processors $(2.93 \mathrm{GHz})$ running Mac OS X® 10.7 .5 (11G63), Kernel Version Darwin 11.4.2. All electronic input/output and spreadsheet files are contained in a zip file that can be accessed by selecting "Additional Information" (select Native File) in the INL Electronic Document Management System. 
TEM-10200-1

$03 / 01 / 2012$

ENGINEERING CALCULATIONS AND ANALYSIS

Page 11 of 30

Rev. 06

Baseline Radionuclide Inventory for the Remote-Handled Low-Level Waste Disposal

Title: $\quad$ Facility for Use in the Facility Performance Assessment

$\begin{array}{lllll}\text { ECAR No.: } 3940 & \text { Rev. No.: } & 0 & \text { Project No.: } 31055 \quad \text { Date: } 01 / 24 / 18\end{array}$

\section{DISCUSSION/ANALYSIS}

Tables in this section contains the 20-year and 50 -year radionuclide inventory by generator, waste form, and vault type to be disposed of in the RH-LLW Disposal Facility.

\section{ATR Inventory}

ATR Activated Metal Inventory for Disposal in HFEF Vaults

Table 5. ATR activated metal 20-year and 50-year inventory estimates for HFEF vaults.

\begin{tabular}{|c|c|c|c|c|c|c|c|c|}
\hline Nuclide & 20-Year & 50-Year & Nuclide & 20-Year & 50-Year & Nuclide & 20-Year & 50-Year \\
\hline $\mathrm{Ag}-108$ & $1.20 \mathrm{E}-06$ & $2.40 \mathrm{E}-06$ & In-114m & $5.55 E-23$ & 1.11E-22 & Sb-124 & 2.61E-15 & $5.22 E-15$ \\
\hline $\mathrm{Ag}-108 \mathrm{~m}$ & $1.29 \mathrm{E}-05$ & $2.58 \mathrm{E}-05$ & In-115 & $2.02 \mathrm{E}-15$ & $4.04 \mathrm{E}-15$ & Sb-125 & $2.04 \mathrm{E}+00$ & $4.08 \mathrm{E}+00$ \\
\hline $\mathrm{Ag}-109 \mathrm{~m}$ & $2.79 \mathrm{E}-04$ & 5.57E-04 & |r-192 & $5.69 \mathrm{E}-15$ & $1.14 \mathrm{E}-14$ & Sc-46 & $1.06 \mathrm{E}-12$ & $2.12 \mathrm{E}-12$ \\
\hline $\mathrm{Ag}-110$ & 1.00E-09 & 2.01E-09 & Ir-192m & $1.05 \mathrm{E}-19$ & $2.10 \mathrm{E}-19$ & Se-75 & 2.46E-07 & 4.91E-07 \\
\hline $\mathrm{Ag}-110 \mathrm{~m}$ & 7.73E-08 & $1.55 \mathrm{E}-07$ & Ir-194 & $1.05 \mathrm{E}-19$ & $2.10 \mathrm{E}-19$ & Se-79 & 1.41E-03 & 2.82E-03 \\
\hline Ar-37 & $1.08 \mathrm{E}-31$ & $2.15 \mathrm{E}-31$ & $\mathrm{~K}-40$ & 7.44E-08 & $1.49 \mathrm{E}-07$ & $\mathrm{Si}-32$ & $2.57 \mathrm{E}-07$ & $5.14 \mathrm{E}-07$ \\
\hline Ar-39 & $5.67 \mathrm{E}-04$ & 1.13E-03 & K-42 & $2.75 \mathrm{E}-12$ & $5.51 \mathrm{E}-12$ & Sn-113 & 1.37E-09 & $2.74 \mathrm{E}-09$ \\
\hline Ar-42 & $2.75 \mathrm{E}-12$ & $5.51 \mathrm{E}-12$ & $K r-81$ & $8.80 \mathrm{E}-13$ & $1.76 \mathrm{E}-12$ & Sn-119m & 1.62E-03 & $3.25 \mathrm{E}-03$ \\
\hline $\mathrm{Ba}-137 \mathrm{~m}$ & $8.95 E-25$ & $1.79 \mathrm{E}-24$ & Kr-85 & 5.79E-07 & 1.16E-06 & $\mathrm{Sn}-121 \mathrm{~m}$ & 1.05E-02 & 2.09E-02 \\
\hline $\mathrm{Be}-10$ & 6.61E-06 & 1.32E-05 & Lu-176 & 2.02E-09 & 4.03E-09 & Sn-123 & $6.90 \mathrm{E}-09$ & $1.38 \mathrm{E}-08$ \\
\hline $\mathrm{Bi}-208$ & $4.92 \mathrm{E}-13$ & $9.85 E-13$ & Lu-177 & 4.03E-09 & 8.05E-09 & Sr-85 & $9.56 \mathrm{E}-20$ & 1.91E-19 \\
\hline $\mathrm{Bi}-210 \mathrm{~m}$ & $3.24 \mathrm{E}-13$ & $6.48 \mathrm{E}-13$ & Lu-177m & 1.87E-08 & $3.75 E-08$ & Sr-89 & 7.44E-24 & 1.49E-23 \\
\hline$C-14$ & $2.36 \mathrm{E}+01$ & $4.71 \mathrm{E}+01$ & Mn-54 & $1.44 \mathrm{E}+01$ & $2.89 \mathrm{E}+01$ & Sr-90 & 3.65E-06 & 7.31E-06 \\
\hline $\mathrm{Ca}-41$ & $3.16 \mathrm{E}-03$ & $6.33 \mathrm{E}-03$ & Mo-93 & 5.41E-01 & $1.08 \mathrm{E}+00$ & Ta-180 & $3.48 \mathrm{E}-01$ & $6.97 \mathrm{E}-01$ \\
\hline $\mathrm{Ca}-45$ & 4.56E-07 & $9.11 \mathrm{E}-07$ & $\mathrm{Nb}-93 \mathrm{~m}$ & 1.87E-04 & 3.74E-04 & Ta-182 & 1.05E-04 & 2.10E-04 \\
\hline Cd-109 & 2.79E-04 & 5.57E-04 & Nb-94 & $3.82 \mathrm{E}+01$ & $7.64 \mathrm{E}+01$ & Tc-99 & 2.58E-02 & 5.17E-02 \\
\hline$C d-115 m$ & $1.97 \mathrm{E}-25$ & $3.94 \mathrm{E}-25$ & $\mathrm{Nb}-95$ & 1.13E-17 & $2.25 \mathrm{E}-17$ & Te-123 & $9.94 \mathrm{E}-11$ & $1.99 \mathrm{E}-10$ \\
\hline Cl-36 & $3.40 \mathrm{E}-06$ & $6.80 \mathrm{E}-06$ & $\mathrm{Nb}-95 \mathrm{~m}$ & 1.79E-18 & $3.58 \mathrm{E}-18$ & Te-123m & 1.71E-07 & $3.41 \mathrm{E}-07$ \\
\hline Co-58 & $1.19 \mathrm{E}-11$ & $2.39 \mathrm{E}-11$ & $\mathrm{Ni}-59$ & $1.90 \mathrm{E}+02$ & $3.81 \mathrm{E}+02$ & $\mathrm{Te}-125 \mathrm{~m}$ & 4.71E-01 & $9.42 \mathrm{E}-01$ \\
\hline Co-60 & $6.90 \mathrm{E}+04$ & $1.38 \mathrm{E}+05$ & $\mathrm{Ni}-63$ & $2.44 \mathrm{E}+04$ & $4.89 \mathrm{E}+04$ & Te-127 & $9.96 \mathrm{E}-14$ & 1.99E-13 \\
\hline Cr-51 & 1.51E-34 & $3.01 \mathrm{E}-34$ & Os-194 & $7.15 \mathrm{E}-16$ & $1.43 E-15$ & $\mathrm{Te}-127 \mathrm{~m}$ & $1.01 \mathrm{E}-13$ & $2.03 \mathrm{E}-13$ \\
\hline Cs-134 & 7.17E-18 & $1.43 \mathrm{E}-17$ & P-32 & $2.57 \mathrm{E}-07$ & $5.14 \mathrm{E}-07$ & TI-204 & 1.10E-22 & 2.19E-22 \\
\hline Cs-135 & $9.18 \mathrm{E}-24$ & $1.84 \mathrm{E}-23$ & $\mathrm{~Pb}-204$ & 2.36E-13 & $4.72 \mathrm{E}-13$ & TI-206 & 3.04E-03 & 6.08E-03 \\
\hline Cs-137 & $9.46 \mathrm{E}-25$ & $1.89 \mathrm{E}-24$ & Pb-205 & $1.15 \mathrm{E}-07$ & $2.31 \mathrm{E}-07$ & Tm-170 & $3.53 \mathrm{E}-21$ & $7.05 \mathrm{E}-21$ \\
\hline $\mathrm{Fe}-55$ & $3.23 E+04$ & $6.46 \mathrm{E}+04$ & Pd-107 & $1.31 \mathrm{E}-14$ & $2.62 \mathrm{E}-14$ & Tm-171 & $3.47 E-15$ & $6.94 \mathrm{E}-15$ \\
\hline Fe-59 & 4.67E-21 & $9.35 \mathrm{E}-21$ & Po-210 & $5.13 \mathrm{E}-15$ & $1.03 \mathrm{E}-14$ & V-50 & $1.25 \mathrm{E}-11$ & $2.51 \mathrm{E}-11$ \\
\hline H-3 & $1.76 \mathrm{E}+03$ & $3.52 E+03$ & Pt-193 & $2.18 \mathrm{E}-12$ & 4.36E-12 & $W-181$ & 3.90E-21 & 7.79E-21 \\
\hline Hf-175 & 4.39E-14 & 8.77E-14 & Rb-87 & $3.22 \mathrm{E}-14$ & $6.44 \mathrm{E}-14$ & W-185 & 5.57E-13 & 1.11E-12 \\
\hline Hf-181 & 2.37E-22 & 4.73E-22 & Re-187 & $1.64 \mathrm{E}-11$ & $3.29 \mathrm{E}-11$ & $W-188$ & $1.05 E-19$ & 2.10E-19 \\
\hline Hf-182 & 1.65E-05 & 3.31E-05 & $\operatorname{Re}-188$ & 1.05E-19 & 2.10E-19 & Y-90 & 3.65E-06 & 7.31E-06 \\
\hline Ho-166m & 8.94E-33 & 1.79E-32 & Rh-106 & 7.33E-19 & $1.47 \mathrm{E}-18$ & Y-91 & $1.58 \mathrm{E}-20$ & 3.16E-20 \\
\hline I-129 & $2.47 \mathrm{E}-15$ & $4.93 E-15$ & Ru-103 & 1.18E-31 & 2.36E-31 & $\mathrm{Zn}-65$ & 2.36E-02 & 4.72E-02 \\
\hline In-113m & 1.37E-09 & 2.74E-09 & Ru-106 & 7.33E-19 & $1.47 \mathrm{E}-18$ & Zr-93 & 4.29E-04 & 8.59E-04 \\
\hline In-114 & $5.30 \mathrm{E}-23$ & $1.06 \mathrm{E}-22$ & S-35 & $6.41 \mathrm{E}-12$ & $1.28 \mathrm{E}-11$ & Zr-95 & $2.23 \mathrm{E}-16$ & $4.47 \mathrm{E}-16$ \\
\hline
\end{tabular}


TEM-10200-1

$03 / 01 / 2012$

Rev. 06

Baseline Radionuclide Inventory for the Remote-Handled Low-Level Waste Disposal

Title: $\quad$ Facility for Use in the Facility Performance Assessment

$\begin{array}{lllll}\text { ECAR No.: } 3940 & \text { Rev. No.: } 0 & \text { Project No.: } 31055 \quad \text { Date: } 01 / 24 / 18\end{array}$

\section{ATR Resin Inventory for Disposal in NuPac Vaults}

Table 6. ATR resin 20-year and 50-year inventory estimates for NuPac vaults.

\begin{tabular}{|c|c|c|c|c|c|c|c|c|}
\hline Nuclide & 20-Year & 50-Year & Nuclide & 20-Year & 50-Year & Nuclide & 20-Year & 50-Year \\
\hline $\mathrm{Ag}-110 \mathrm{~m}$ & 2.13E-02 & 5.33E-02 & I-133 & 8.66E-03 & 2.16E-02 & Ru-106 & $1.28 \mathrm{E}-01$ & 3.20E-01 \\
\hline Am-241 & 1.34E-02 & 3.34E-02 & In-113m & 3.07E-02 & 7.67E-02 & Sb-124 & 1.94E-01 & 4.84E-01 \\
\hline As-76 & 8.28E-03 & 2.07E-02 & In-114 & 3.07E-05 & 7.67E-05 & Sb-125 & 9.36E-02 & 2.34E-01 \\
\hline $\mathrm{Ba}-137 \mathrm{~m}$ & $3.46 \mathrm{E}+00$ & $8.65 E+00$ & In-114m & $3.20 \mathrm{E}-05$ & 8.01E-05 & Sc-46 & 2.39E-01 & 5.99E-01 \\
\hline $\mathrm{Ba}-140$ & $5.24 \mathrm{E}-02$ & $1.31 \mathrm{E}-01$ & $K-40$ & 5.06E-04 & $1.26 \mathrm{E}-03$ & Se-75 & 3.23E-02 & 8.08E-02 \\
\hline C-14 & $9.77 \mathrm{E}-01$ & $2.44 \mathrm{E}+00$ & La-140 & $1.18 \mathrm{E}-01$ & $2.96 \mathrm{E}-01$ & Si-32 & 7.83E-09 & 1.96E-08 \\
\hline $\mathrm{Ca}-45$ & $9.25 \mathrm{E}-04$ & $2.31 \mathrm{E}-03$ & Mn-54 & $2.22 \mathrm{E}+01$ & $5.55 \mathrm{E}+01$ & Sn-113 & 3.07E-02 & 7.67E-02 \\
\hline Cd-109 & $1.28 \mathrm{E}-02$ & $3.20 \mathrm{E}-02$ & Mo-99 & 4.42E-06 & 1.10E-05 & Sn-117m & 4.97E-06 & $1.24 \mathrm{E}-05$ \\
\hline Ce-141 & $5.79 \mathrm{E}-01$ & $1.45 \mathrm{E}+00$ & $\mathrm{Na}-24$ & $3.68 \mathrm{E}-06$ & $9.21 \mathrm{E}-06$ & Sn-119m & 3.34E-01 & 8.35E-01 \\
\hline $\mathrm{Ce}-144$ & $2.79 \mathrm{E}+01$ & $6.97 \mathrm{E}+01$ & $\mathrm{Nb}-93 \mathrm{~m}$ & 7.83E-09 & 1.96E-08 & Sn-123 & 1.28E-02 & $3.20 \mathrm{E}-02$ \\
\hline $\mathrm{Cm}-242$ & 1.70E-03 & $4.25 \mathrm{E}-03$ & $\mathrm{Nb}-94$ & 8.48E-01 & $2.12 \mathrm{E}+00$ & Sr-89 & $9.25 \mathrm{E}-06$ & $2.31 \mathrm{E}-05$ \\
\hline $\mathrm{Cm}-244$ & $9.38 \mathrm{E}-03$ & $2.34 \mathrm{E}-02$ & $\mathrm{Nb}-95$ & $4.90 \mathrm{E}+00$ & $1.22 \mathrm{E}+01$ & Sr-90 & $1.62 \mathrm{E}+01$ & $4.05 E+01$ \\
\hline Co-57 & $3.14 \mathrm{E}-04$ & 7.84E-04 & $\mathrm{Nb}-95 \mathrm{~m}$ & 1.14E-04 & 2.84E-04 & Ta-182 & 2.22E-02 & 5.54E-02 \\
\hline Co-58 & $4.64 \mathrm{E}+01$ & $1.16 \mathrm{E}+02$ & $\mathrm{Ni}-59$ & 7.61E-01 & $1.90 \mathrm{E}+00$ & Tc-99 & $1.97 \mathrm{E}+00$ & $4.93 \mathrm{E}+00$ \\
\hline Co-60 & $2.29 \mathrm{E}+02$ & $5.73 E+02$ & $\mathrm{Ni}-63$ & $3.40 \mathrm{E}+01$ & $8.49 \mathrm{E}+01$ & $\mathrm{Te}-123 \mathrm{~m}$ & 1.23E-06 & 3.08E-06 \\
\hline Cr-51 & $6.25 \mathrm{E}+02$ & $1.56 \mathrm{E}+03$ & Np-237 & $9.18 \mathrm{E}-05$ & $2.29 \mathrm{E}-04$ & $\mathrm{Te}-125 \mathrm{~m}$ & 2.13E-02 & 5.33E-02 \\
\hline Cs-134 & $6.79 \mathrm{E}-01$ & $1.70 \mathrm{E}+00$ & P-32 & $1.42 \mathrm{E}-08$ & $3.55 E-08$ & Te-127 & 5.84E-09 & 1.46E-08 \\
\hline Cs-137 & $6.14 \mathrm{E}+00$ & $1.54 \mathrm{E}+01$ & Pm-147 & $3.36 \mathrm{E}+01$ & $8.40 \mathrm{E}+01$ & Te-127m & 5.97E-09 & 1.49E-08 \\
\hline Eu-152 & 5.34E-01 & $1.34 \mathrm{E}+00$ & Pr-144 & $1.43 \mathrm{E}+01$ & $3.57 \mathrm{E}+01$ & Te-132 & 7.94E-08 & $1.99 \mathrm{E}-07$ \\
\hline Eu-154 & $6.58 \mathrm{E}-01$ & $1.64 \mathrm{E}+00$ & Pu-238 & 1.09E-02 & $2.72 \mathrm{E}-02$ & Th-228 & 1.06E-06 & 2.64E-06 \\
\hline Eu-155 & $2.13 \mathrm{E}-01$ & 5.32E-01 & Pu-239 & $2.88 \mathrm{E}-02$ & 7.20E-02 & U-234 & $9.18 \mathrm{E}-05$ & $2.29 \mathrm{E}-04$ \\
\hline Fe-55 & $3.99 \mathrm{E}+02$ & $9.97 \mathrm{E}+02$ & Pu-240 & $1.81 \mathrm{E}-03$ & $4.52 \mathrm{E}-03$ & U-235 & 4.53E-06 & 1.13E-05 \\
\hline Fe-59 & $1.83 \mathrm{E}+01$ & $4.58 \mathrm{E}+01$ & Pu-241 & $1.52 \mathrm{E}-03$ & 3.80E-03 & W-187 & 3.58E-01 & 8.94E-01 \\
\hline $\mathrm{H}-3$ & 1.09E-01 & $2.73 \mathrm{E}-01$ & $\operatorname{Re}-186$ & $2.54 \mathrm{E}-06$ & 6.35E-06 & $Y-90$ & $1.50 \mathrm{E}+01$ & $3.74 \mathrm{E}+01$ \\
\hline Hf-175 & 7.19E-02 & $1.80 \mathrm{E}-01$ & Re-187 & $3.58 \mathrm{E}-01$ & 8.94E-01 & Y-91 & $3.55 \mathrm{E}-05$ & 8.87E-05 \\
\hline Hf-181 & 8.66E-01 & $2.17 \mathrm{E}+00$ & $\operatorname{Re}-188$ & $2.45 \mathrm{E}-01$ & $6.12 \mathrm{E}-01$ & $\mathrm{Zn}-65$ & $3.25 E+01$ & $8.11 \mathrm{E}+01$ \\
\hline |-129 & 5.33E-02 & 1.33E-01 & Rh-103m & 9.79E-03 & $2.45 \mathrm{E}-02$ & $Z r-93$ & 1.83E-02 & 4.57E-02 \\
\hline |-131 & 1.37E-02 & $3.42 \mathrm{E}-02$ & Rh-106 & $1.28 \mathrm{E}-01$ & $3.20 \mathrm{E}-01$ & Zr-95 & $3.14 \mathrm{E}+00$ & $7.86 \mathrm{E}+00$ \\
\hline |-132 & $2.18 \mathrm{E}-03$ & $5.46 \mathrm{E}-03$ & Ru-103 & 2.37E-01 & 5.92E-01 & & & \\
\hline
\end{tabular}


TEM-10200-1

$03 / 01 / 2012$

ENGINEERING CALCULATIONS AND ANALYSIS

Page 13 of 30

Rev. 06

Baseline Radionuclide Inventory for the Remote-Handled Low-Level Waste Disposal

Title: $\quad$ Facility for Use in the Facility Performance Assessment

$\begin{array}{lllll}\text { ECAR No.: } 3940 & \text { Rev. No.: } 0 & \text { Project No.: } 31055 \quad \text { Date: } 01 / 24 / 18\end{array}$

\section{MFC Inventory}

MFC Legacy Activated Metal and Surface Contamination Inventory for Disposal in MFTC Vaults

Table 7. MFC legacy activated metal (AM) and surface contamination (SC) 20-year and 50-year inventory estimates for MFTC vaults.

\begin{tabular}{|c|c|c|c|c|c|c|c|}
\hline Nuclide & $\begin{array}{l}\text { Waste } \\
\text { Form }\end{array}$ & $20-$ Year & 50 -Year & Nuclide & $\begin{array}{c}\text { Waste } \\
\text { Form }\end{array}$ & 20 -Year & 50-Year \\
\hline C-14 & AM & $1.95 E+01$ & $1.95 E+01$ & Pd-107 & $S C^{b}$ & 4.35E-04 & 4.35E-04 \\
\hline Co-60 & AM & $1.43 E+03$ & $1.43 E+03$ & Pm-147 & $S C^{b}$ & $1.23 \mathrm{E}-02$ & 1.23E-02 \\
\hline $\mathrm{Fe}-55$ & AM & $2.52 \mathrm{E}+00$ & $2.52 \mathrm{E}+00$ & Ru-106 & $S C^{b}$ & $9.36 \mathrm{E}-13$ & $9.36 \mathrm{E}-13$ \\
\hline Mn-54 & AM & 7.74E-12 & 7.74E-12 & Sb-125 & $S C^{b}$ & 3.69E-03 & $3.69 \mathrm{E}-03$ \\
\hline Mo-93 & AM & $2.17 E+01$ & $2.17 E+01$ & Se-79 & $S C^{b}$ & 7.82E-03 & 7.82E-03 \\
\hline $\mathrm{Nb}-94$ & AM & $4.74 \mathrm{E}+00$ & $4.74 \mathrm{E}+00$ & Sm-151 & $S C^{b}$ & $5.24 \mathrm{E}+01$ & $5.24 \mathrm{E}+01$ \\
\hline $\mathrm{Ni}-59$ & AM & $9.05 E+01$ & $9.05 E+01$ & Sn-119m & $S C^{b}$ & 1.37E-17 & $1.37 \mathrm{E}-17$ \\
\hline $\mathrm{Ni}-63$ & AM & $4.60 E+03$ & $4.60 E+03$ & Sr-90 & $S C^{b}$ & $6.35 E+02$ & $6.35 E+02$ \\
\hline C-14 & $S C^{a}$ & 2.87E-01 & 2.87E-01 & Tc-99 & $S C^{b}$ & $2.57 E+00$ & $2.57 \mathrm{E}+00$ \\
\hline Co-57 & $S C^{a}$ & 4.74E-22 & 4.74E-22 & Zr-93 & $S C^{b}$ & 7.09E-02 & 7.09E-02 \\
\hline Co-60 & $\mathrm{SC}^{\mathrm{a}}$ & $1.68 E+01$ & $1.68 E+01$ & Am-241 & $S C^{c}$ & 2.61E-05 & 2.61E-05 \\
\hline Fe-55 & $S C^{a}$ & $2.19 \mathrm{E}-02$ & $2.19 \mathrm{E}-02$ & Np-237 & $S C^{c}$ & 5.82E-04 & 5.82E-04 \\
\hline$M n-54$ & $S C^{a}$ & 8.07E-15 & 8.07E-15 & $\mathrm{Pb}-210$ & $S C^{c}$ & $2.89 \mathrm{E}-12$ & $2.89 \mathrm{E}-12$ \\
\hline Mo-93 & $S C^{a}$ & 3.19E-01 & 3.19E-01 & Pu-238 & $S C^{c}$ & 2.92E-02 & $2.92 \mathrm{E}-02$ \\
\hline $\mathrm{Nb}-94$ & $S C^{a}$ & 7.02E-02 & 7.02E-02 & Pu-239 & $S C^{c}$ & $2.99 \mathrm{E}-01$ & $2.99 \mathrm{E}-01$ \\
\hline $\mathrm{Ni}-59$ & $S C^{a}$ & $1.33 E+00$ & $1.33 E+00$ & Pu-240 & $S C^{c}$ & $1.85 \mathrm{E}-03$ & $1.85 \mathrm{E}-03$ \\
\hline $\mathrm{Ni}-63$ & $\mathrm{SC}^{\mathrm{a}}$ & $6.70 \mathrm{E}+01$ & $6.70 \mathrm{E}+01$ & Pu-241 & $S C^{c}$ & $8.41 \mathrm{E}-07$ & $8.41 \mathrm{E}-07$ \\
\hline Ce-144 & $S C^{b}$ & $5.41 \mathrm{E}-08$ & $5.41 \mathrm{E}-08$ & Pu-242 & $S C^{c}$ & $1.41 \mathrm{E}-06$ & $1.41 \mathrm{E}-06$ \\
\hline Cs-134 & $S C^{b}$ & 2.27E-04 & $2.27 \mathrm{E}-04$ & Pu-244 & $S C^{c}$ & $7.02 \mathrm{E}-23$ & $7.02 \mathrm{E}-23$ \\
\hline Cs-135 & $S C^{b}$ & $2.60 \mathrm{E}-02$ & $2.60 \mathrm{E}-02$ & $\mathrm{Ra}-226$ & $S C^{c}$ & $9.53 \mathrm{E}-12$ & $9.53 \mathrm{E}-12$ \\
\hline Cs-137 & $S C^{b}$ & $9.14 \mathrm{E}+02$ & $9.14 \mathrm{E}+02$ & Th-229 & $S C^{c}$ & $8.13 \mathrm{E}-17$ & $8.13 \mathrm{E}-17$ \\
\hline Eu-154 & $S C^{b}$ & $3.29 \mathrm{E}+00$ & $3.29 \mathrm{E}+00$ & Th-230 & $S C^{c}$ & 1.26E-09 & 1.26E-09 \\
\hline Eu-155 & $S C^{b}$ & $1.48 \mathrm{E}-01$ & $1.48 \mathrm{E}-01$ & U-233 & $\mathrm{SC}^{\mathrm{c}}$ & $5.04 \mathrm{E}-14$ & $5.04 \mathrm{E}-14$ \\
\hline I-129 & $S C^{b}$ & 4.83E-04 & 4.83E-04 & U-234 & $S C^{c}$ & 5.16E-06 & 5.16E-06 \\
\hline In-115 & $S C^{b}$ & $7.52 \mathrm{E}-13$ & $7.52 \mathrm{E}-13$ & U-235 & $S C^{c}$ & $3.70 \mathrm{E}-03$ & 3.70E-03 \\
\hline Kr-85 & $S C^{b}$ & $1.40 E+01$ & $1.40 \mathrm{E}+01$ & U-238 & $S C^{c}$ & $7.40 \mathrm{E}-04$ & 7.40E-04 \\
\hline
\end{tabular}

a. Activation product as SC.

b. Fission product as SC.

c. Other isotopic activity as SC. 
TEM-10200-1

$03 / 01 / 2012$

Rev. 06

Baseline Radionuclide Inventory for the Remote-Handled Low-Level Waste Disposal

Title: $\quad$ Facility for Use in the Facility Performance Assessment

$\begin{array}{lllll}\text { ECAR No.: } 3940 \quad \text { Rev. No.: } & 0 & \text { Project No.: } 31055 \quad \text { Date: } 01 / 24 / 18\end{array}$

MFC Legacy Activated Metal and Surface Contamination Inventory for Disposal in HFEF Vaults

Table 8. MFC legacy AM and SC 20-year and 50-year inventory estimates for HFEF vaults.

\begin{tabular}{|c|c|c|c|c|c|c|c|}
\hline Nuclide & $\begin{array}{l}\text { Waste } \\
\text { Form }\end{array}$ & 20-Year & 50-year & Nuclide & $\begin{array}{l}\text { Waste } \\
\text { Form }\end{array}$ & 20-Year & 50 -Year \\
\hline$C-14$ & $\mathrm{AM}$ & $1.16 \mathrm{E}+00$ & $1.16 \mathrm{E}+00$ & Sn-123 & $S C^{b}$ & $6.74 \mathrm{E}-13$ & $6.74 \mathrm{E}-13$ \\
\hline Co-57 & AM & $2.46 \mathrm{E}-12$ & $2.46 \mathrm{E}-12$ & Sn-126 & $\mathrm{SC}^{\mathrm{b}}$ & $1.94 \mathrm{E}-03$ & $1.94 \mathrm{E}-03$ \\
\hline Co-58 & AM & 9.34E-07 & 9.34E-07 & Sr-85 & $\mathrm{SC}^{\mathrm{b}}$ & $6.08 \mathrm{E}-15$ & $6.08 \mathrm{E}-15$ \\
\hline Co-60 & AM & $1.08 \mathrm{E}+04$ & $1.08 \mathrm{E}+04$ & Sr-90 & $S C^{b}$ & $5.13 E+00$ & $5.13 E+00$ \\
\hline Cr-51 & AM & $7.19 \mathrm{E}-20$ & $7.19 \mathrm{E}-20$ & Tc-98 & $S C^{b}$ & 7.08E-09 & $7.08 \mathrm{E}-09$ \\
\hline $\mathrm{Fe}-55$ & AM & $6.84 \mathrm{E}+02$ & $6.84 \mathrm{E}+02$ & Tc-99 & $S C^{b}$ & $1.90 \mathrm{E}-01$ & $1.90 \mathrm{E}-01$ \\
\hline Fe-59 & AM & 1.10E-12 & 1.10E-12 & Te-125m & $\mathrm{SC}^{\mathrm{b}}$ & $1.22 \mathrm{E}-20$ & $1.22 \mathrm{E}-20$ \\
\hline $\mathrm{H}-3$ & AM & $1.25 \mathrm{E}+00$ & $1.25 \mathrm{E}+00$ & Te-127m & $S C^{b}$ & $5.72 \mathrm{E}-15$ & $5.72 \mathrm{E}-15$ \\
\hline Mn-54 & AM & $5.52 E+00$ & $5.52 \mathrm{E}+00$ & Zr-93 & $\mathrm{SC}^{\mathrm{b}}$ & 2.67E-03 & 2.67E-03 \\
\hline Mo-93 & AM & $2.27 \mathrm{E}+00$ & $2.27 \mathrm{E}+00$ & Zr-95 & $S C^{b}$ & $9.45 \mathrm{E}-24$ & $9.45 \mathrm{E}-24$ \\
\hline $\mathrm{Nb}-94$ & AM & 4.26E-01 & 4.26E-01 & Ac-227 & $\mathrm{SC}^{\mathrm{c}}$ & 1.21E-07 & $1.21 \mathrm{E}-07$ \\
\hline $\mathrm{Ni}-59$ & AM & $3.28 \mathrm{E}+00$ & $3.28 \mathrm{E}+00$ & $A m-241$ & $S C^{c}$ & $6.22 \mathrm{E}-03$ & $6.22 \mathrm{E}-03$ \\
\hline $\mathrm{Ni}-63$ & AM & $2.87 E+02$ & $2.87 \mathrm{E}+02$ & $\mathrm{Be}-10$ & $S C^{c}$ & 4.96E-07 & 4.96E-07 \\
\hline Co-57 & $S C^{a}$ & 1.13E-07 & 1.13E-07 & Ir-192 & $S C^{c}$ & $1.29 E-25$ & $1.29 \mathrm{E}-25$ \\
\hline Co-58 & $\mathrm{SC}^{\mathrm{a}}$ & $4.95 \mathrm{E}-14$ & $4.95 \mathrm{E}-14$ & Np-237 & $\mathrm{SC}^{\mathrm{c}}$ & 6.86E-08 & $6.86 \mathrm{E}-08$ \\
\hline Co-60 & $S C^{a}$ & 7.90E-01 & 7.90E-01 & $\mathrm{Pb}-210$ & $S C^{c}$ & $4.68 \mathrm{E}-17$ & $4.68 \mathrm{E}-17$ \\
\hline $\mathrm{H}-3$ & $\mathrm{SC}^{\mathrm{a}}$ & $3.49 \mathrm{E}-05$ & $3.49 \mathrm{E}-05$ & Pt-193 & $\mathrm{SC}^{\mathrm{C}}$ & $1.51 \mathrm{E}-10$ & $1.51 \mathrm{E}-10$ \\
\hline Mn-54 & $S C^{a}$ & 4.91E-08 & 4.91E-08 & Pu-236 & $\mathrm{SC}^{\mathrm{c}}$ & 1.77E-07 & 1.77E-07 \\
\hline $\mathrm{Ag}-108 \mathrm{~m}$ & $S C^{b}$ & 1.03E-07 & 1.03E-07 & Pu-238 & $\mathrm{SC}^{\mathrm{c}}$ & 1.38E-04 & $1.38 \mathrm{E}-04$ \\
\hline $\mathrm{Ag}-110 \mathrm{~m}$ & $S C^{b}$ & 1.75E-08 & 1.75E-08 & Pu-239 & $\mathrm{SC}^{\mathrm{c}}$ & 9.05E-03 & $9.05 \mathrm{E}-03$ \\
\hline Cd-113m & $S C^{b}$ & $2.22 \mathrm{E}-02$ & 2.22E-02 & Pu-240 & $\mathrm{SC}^{\mathrm{C}}$ & 5.40E-05 & 5.40E-05 \\
\hline Ce-144 & $S C^{b}$ & $4.38 \mathrm{E}-06$ & 4.38E-06 & Pu-241 & $\mathrm{SC}^{\mathrm{C}}$ & 2.33E-04 & $2.33 E-04$ \\
\hline Cs-134 & $S C^{b}$ & 5.78E-04 & 5.78E-04 & Pu-242 & $S C^{c}$ & 1.23E-08 & $1.23 \mathrm{E}-08$ \\
\hline Cs-137 & $S C^{b}$ & $2.49 \mathrm{E}+00$ & $2.49 \mathrm{E}+00$ & Ra-226 & $\mathrm{SC}^{\mathrm{C}}$ & $4.19 \mathrm{E}-16$ & $4.19 \mathrm{E}-16$ \\
\hline Eu-154 & $\mathrm{SC}^{\mathrm{b}}$ & 1.13E-03 & 1.13E-03 & Re-187 & $\mathrm{SC}^{\mathrm{C}}$ & $1.55 \mathrm{E}-07$ & $1.55 \mathrm{E}-07$ \\
\hline Eu-155 & $S C^{b}$ & $3.50 \mathrm{E}-03$ & $3.50 \mathrm{E}-03$ & Sc-46 & $\mathrm{SC}^{\mathrm{c}}$ & 1.55E-22 & $1.55 \mathrm{E}-22$ \\
\hline I-129 & $S C^{b}$ & 4.40E-09 & 4.40E-09 & Ta-182 & $\mathrm{SC}^{\mathrm{c}}$ & 5.65E-15 & 5.65E-15 \\
\hline $\mathrm{Kr}-85$ & $\mathrm{SC}^{\mathrm{b}}$ & $1.43 E+00$ & $1.43 E+00$ & Th-229 & $\mathrm{SC}^{\mathrm{c}}$ & $1.66 \mathrm{E}-15$ & $1.66 \mathrm{E}-15$ \\
\hline $\mathrm{Nb}-93 \mathrm{~m}$ & $S C^{b}$ & 1.27E-01 & 1.27E-01 & Th-230 & $\mathrm{SC}^{\mathrm{c}}$ & $2.15 \mathrm{E}-13$ & $2.15 \mathrm{E}-13$ \\
\hline Pm-147 & $S C^{b}$ & $1.08 \mathrm{E}-04$ & $1.08 \mathrm{E}-04$ & U-232 & $S C^{c}$ & 3.44E-07 & $3.44 \mathrm{E}-07$ \\
\hline Rh-102 & $S C^{b}$ & 1.10E-10 & $1.10 \mathrm{E}-10$ & U-233 & $S C^{c}$ & 3.38E-06 & $3.38 \mathrm{E}-06$ \\
\hline Ru-106 & $S C^{b}$ & 4.04E-03 & 4.04E-03 & U-234 & $S C^{c}$ & 2.69E-05 & $2.69 \mathrm{E}-05$ \\
\hline Sb-124 & $S C^{b}$ & 1.80E-21 & 1.80E-21 & U-235 & $S C^{c}$ & 3.07E-07 & 3.07E-07 \\
\hline Sb-125 & $S C^{b}$ & $1.02 \mathrm{E}-01$ & $1.02 \mathrm{E}-01$ & U-236 & $\mathrm{SC}^{\mathrm{C}}$ & 5.02E-07 & 5.02E-07 \\
\hline Sm-151 & $\mathrm{SC}^{\mathrm{b}}$ & 4.17E-04 & 4.17E-04 & $\mathrm{U}-238$ & $\mathrm{SC}^{\mathrm{c}}$ & 2.01E-07 & $2.01 \mathrm{E}-07$ \\
\hline Sn-119m & $S C^{b}$ & 1.75E-07 & 1.75E-07 & $V-49$ & $S C^{c}$ & $2.10 \mathrm{E}-11$ & $2.10 \mathrm{E}-11$ \\
\hline
\end{tabular}

\footnotetext{
a. Activation product as SC.

b. Fission product as SC.

c. Other isotopic activity as SC.
} 
TEM-10200-1

$03 / 01 / 2012$

Rev. 06

ENGINEERING CALCULATIONS AND ANALYSIS

Page 15 of 30

Baseline Radionuclide Inventory for the Remote-Handled Low-Level Waste Disposal

Title: $\quad$ Facility for Use in the Facility Performance Assessment

$\begin{array}{lllll}\text { ECAR No.: } 3940 & \text { Rev. No.: } & 0 & \text { Project No.: } 31055 \quad \text { Date: } 01 / 24 / 18\end{array}$

\section{MFC Future Generation Activated Metal and Surface Contamination Inventory for Disposal in HFEF Vaults}

Table 9. MFC future generation AM and SC 20-year and 50-year inventory estimates for HFEF vaults.

\begin{tabular}{|c|c|c|c|c|c|c|c|}
\hline Nuclide & $\begin{array}{l}\text { Waste } \\
\text { Form }\end{array}$ & 20-Year & 50-Year & Nuclide & $\begin{array}{l}\text { Waste } \\
\text { Form }\end{array}$ & 20-Year & 50-Year \\
\hline C-14 & AM & 1.59E+00 & $4.00 \mathrm{E}+00$ & Sb-126 & $S C^{b}$ & 6.77E-03 & $1.71 \mathrm{E}-02$ \\
\hline Co-57 & AM & 4.04E-04 & 1.02E-03 & $\mathrm{Sb}-126 \mathrm{~m}$ & $S C^{b}$ & $5.03 E+00$ & $1.27 \mathrm{E}+01$ \\
\hline Co-58 & AM & $7.29 \mathrm{E}+03$ & $1.84 \mathrm{E}+04$ & Sm-151 & $S C^{b}$ & $1.59 \mathrm{E}-07$ & 4.01E-07 \\
\hline Co-60 & AM & $1.25 E+05$ & $3.15 E+05$ & Sn-119m & $S C^{b}$ & 2.10E-02 & 5.29E-02 \\
\hline Cr-51 & AM & $4.89 \mathrm{E}+03$ & $1.23 E+04$ & Sn-123 & $S C^{b}$ & 3.78E-03 & $9.51 \mathrm{E}-03$ \\
\hline $\mathrm{Fe}-55$ & AM & $4.18 E+04$ & $1.05 E+05$ & Sn-126 & $S C^{b}$ & 4.85E-02 & $1.22 \mathrm{E}-01$ \\
\hline Fe-59 & AM & $5.87 \mathrm{E}+02$ & $1.48 \mathrm{E}+03$ & Sr-85 & $S C^{b}$ & 1.93E-04 & 4.87E-04 \\
\hline $\mathrm{H}-3$ & AM & $1.09 E+01$ & $2.73 E+01$ & Sr-90 & $S C^{b}$ & $1.65 E+00$ & $4.16 \mathrm{E}+00$ \\
\hline$M n-54$ & AM & $1.82 E+04$ & $4.59 E+04$ & Tc-98 & $S C^{b}$ & 1.16E-07 & 2.93E-07 \\
\hline Mo-93 & AM & 5.12E-01 & $1.29 \mathrm{E}+00$ & Tc-99 & $S C^{b}$ & 3.46E-01 & 8.73E-01 \\
\hline $\mathrm{Nb}-94$ & AM & 6.84E-01 & $1.72 E+00$ & Te-125m & $S C^{b}$ & $1.22 \mathrm{E}+00$ & $3.08 \mathrm{E}+00$ \\
\hline $\mathrm{Ni}-59$ & AM & $5.57 \mathrm{E}+00$ & $1.40 E+01$ & Te-127 & $S C^{b}$ & 1.10E-03 & 2.77E-03 \\
\hline $\mathrm{Ni}-63$ & AM & $6.46 \mathrm{E}+02$ & $1.63 E+03$ & Te-127m & $S C^{b}$ & 1.13E-03 & $2.85 \mathrm{E}-03$ \\
\hline Co-57 & $\mathrm{SC}^{\mathrm{a}}$ & 7.16E-04 & $1.80 \mathrm{E}-03$ & $\mathrm{Y}-90$ & $S C^{b}$ & $1.65 E+00$ & $4.16 \mathrm{E}+00$ \\
\hline Co-58 & $\mathrm{SC}^{\mathrm{a}}$ & 1.99E-04 & $5.01 \mathrm{E}-04$ & $\mathrm{Zr}-93$ & $S C^{b}$ & 3.34E-02 & 8.42E-02 \\
\hline Co-60 & $S C^{a}$ & 9.54E-04 & $2.40 \mathrm{E}-03$ & Zr-95 & $S C^{b}$ & 8.61E-06 & 2.17E-05 \\
\hline$M n-54$ & $\mathrm{SC}^{\mathrm{a}}$ & 1.19E-04 & 3.01E-04 & Ac-227 & $S C^{c}$ & 4.13E-06 & 1.04E-05 \\
\hline$A g-108$ & $S C^{b}$ & 2.12E-07 & 5.34E-07 & Am-241 & $S C^{c}$ & $9.38 \mathrm{E}-03$ & 2.36E-02 \\
\hline $\mathrm{Ag}-108 \mathrm{~m}$ & $S C^{b}$ & $2.72 \mathrm{E}-06$ & $6.87 \mathrm{E}-06$ & $\mathrm{Be}-10$ & $\mathrm{SC}^{\mathrm{C}}$ & $8.45 \mathrm{E}-06$ & 2.13E-05 \\
\hline $\mathrm{Ag}-110$ & $S C^{b}$ & $1.26 \mathrm{E}-04$ & $3.18 \mathrm{E}-04$ & Bi-211 & $\mathrm{SC}^{\mathrm{c}}$ & 4.17E-06 & $1.05 \mathrm{E}-05$ \\
\hline $\mathrm{Ag}-110 \mathrm{~m}$ & $S C^{b}$ & 9.10E-03 & $2.29 \mathrm{E}-02$ & Bi-212 & $S C^{c}$ & 1.98E-04 & 5.00E-04 \\
\hline Ba-137m & $S C^{b}$ & 1.35E-02 & $3.40 \mathrm{E}-02$ & Ir-192 & $\mathrm{SC}^{\mathrm{c}}$ & 1.25E-09 & 3.14E-09 \\
\hline Cd-113m & $S C^{b}$ & 9.15E-01 & $2.31 E+00$ & $\mathrm{~Pa}-233$ & $S C^{c}$ & 2.07E-17 & $5.21 \mathrm{E}-17$ \\
\hline $\mathrm{Ce}-141$ & $S C^{b}$ & 2.16E-05 & 5.44E-05 & $\mathrm{Pb}-211$ & $S C^{c}$ & 4.15E-06 & $1.05 \mathrm{E}-05$ \\
\hline $\mathrm{Ce}-144$ & $S C^{b}$ & 8.75E-03 & $2.21 \mathrm{E}-02$ & $\mathrm{~Pb}-212$ & $\mathrm{SC}^{\mathrm{c}}$ & 1.98E-04 & 5.00E-04 \\
\hline Cs-134 & $S C^{b}$ & 8.09E-03 & $2.04 \mathrm{E}-02$ & Pt-193 & $\mathrm{SC}^{\mathrm{c}}$ & 2.82E-09 & 7.12E-09 \\
\hline Cs-137 & $S C^{b}$ & 8.17E-01 & $2.06 E+00$ & Pu-238 & $\mathrm{SC}^{\mathrm{C}}$ & 2.63E-05 & $6.62 \mathrm{E}-05$ \\
\hline Eu-154 & $S C^{b}$ & 7.63E-03 & 1.92E-02 & Pu-239 & $\mathrm{SC}^{\mathrm{C}}$ & $6.60 \mathrm{E}-03$ & 1.66E-02 \\
\hline Eu-155 & $S C^{b}$ & 4.66E-03 & 1.18E-02 & $\mathrm{Pu}-240$ & $S C^{c}$ & 7.08E-06 & $1.78 \mathrm{E}-05$ \\
\hline $\mathrm{I}-129$ & $S C^{b}$ & 3.73E-13 & $9.39 \mathrm{E}-13$ & Pu-241 & $S C^{c}$ & 7.11E-05 & 1.79E-04 \\
\hline$K r-85$ & $S C^{b}$ & $3.36 E+01$ & $8.47 E+01$ & Pu-242 & $\mathrm{SC}^{\mathrm{C}}$ & 4.77E-09 & $1.20 \mathrm{E}-08$ \\
\hline $\mathrm{Nb}-93 \mathrm{~m}$ & $S C^{b}$ & 1.16E-05 & 2.92E-05 & $\mathrm{Re}-187$ & $\mathrm{SC}^{\mathrm{c}}$ & $2.55 \mathrm{E}-06$ & $6.43 \mathrm{E}-06$ \\
\hline $\mathrm{Nb}-95$ & $S C^{b}$ & $4.85 E+01$ & $1.22 E+02$ & Sc-46 & $\mathrm{SC}^{\mathrm{C}}$ & 2.87E-08 & $7.24 \mathrm{E}-08$ \\
\hline $\mathrm{Nb}-95 \mathrm{~m}$ & $S C^{b}$ & 3.65E-07 & $9.20 \mathrm{E}-07$ & Ta-182 & $\mathrm{SC}^{\mathrm{C}}$ & 1.70E-04 & 4.28E-04 \\
\hline Pm-147 & $S C^{b}$ & 1.16E-07 & 2.92E-07 & Th-231 & $S C^{c}$ & $4.78 \mathrm{E}-11$ & $1.20 \mathrm{E}-10$ \\
\hline Pr-144 & $S C^{b}$ & 7.35E-03 & 1.85E-02 & TI-207 & $S C^{c}$ & 4.17E-06 & $1.05 \mathrm{E}-05$ \\
\hline Rh-102 & $S C^{b}$ & 1.74E-08 & 4.39E-08 & TI-208 & $\mathrm{SC}^{\mathrm{C}}$ & $7.25 \mathrm{E}-05$ & 1.83E-04 \\
\hline Rh-106 & $S C^{b}$ & $3.44 \mathrm{E}+01$ & $8.66 E+01$ & U-234 & $S C^{c}$ & 9.04E-05 & $2.28 \mathrm{E}-04$ \\
\hline Ru-106 & $S C^{b}$ & $4.30 \mathrm{E}+01$ & $1.08 E+02$ & U-235 & $\mathrm{SC}^{\mathrm{C}}$ & 1.50E-06 & $3.79 \mathrm{E}-06$ \\
\hline
\end{tabular}


TEM-10200-1

$03 / 01 / 2012$

ENGINEERING CALCULATIONS AND ANALYSIS

Page 16 of 30

Rev. 06

Baseline Radionuclide Inventory for the Remote-Handled Low-Level Waste Disposal

Title: $\quad$ Facility for Use in the Facility Performance Assessment

$\begin{array}{lllll}\text { ECAR No.: } 3940 \quad \text { Rev. No.: } & 0 & \text { Project No.: } 31055 \quad \text { Date: } 01 / 24 / 18\end{array}$

Table 9. (continued).

\begin{tabular}{|c|c|c|c|c|c|c|c|}
\hline Nuclide & $\begin{array}{c}\text { Waste } \\
\text { Form }\end{array}$ & 20-Year & 50-Year & Nuclide & $\begin{array}{l}\text { Waste } \\
\text { Form }\end{array}$ & 20-Year & 50-Year \\
\hline Sb-124 & $S C^{b}$ & 1.39E-04 & $3.50 \mathrm{E}-04$ & U-236 & $S C^{c}$ & $1.80 \mathrm{E}-06$ & 4.54E-06 \\
\hline Sb-125 & $S C^{b}$ & $1.28 \mathrm{E}+00$ & $3.24 E+00$ & U-238 & $S C^{c}$ & 7.11E-07 & 1.79E-06 \\
\hline
\end{tabular}

\section{NRF Inventory}

\section{NRF Activated Metal Inventory for Disposal in 55-Ton Vaults}

Table 10. NRF activated metal 20-year and 50-year inventory estimates for 55-ton vaults.

\begin{tabular}{|c|c|c|c|c|c|c|c|c|}
\hline Nuclide & 20-Year & 50-Year & Nuclide & 20-Year & 50-Year & Nuclide & 20-year & 50-Year \\
\hline Ac-225 & 2.51E-09 & 2.51E-09 & Ir-192 & 1.92E-02 & 1.92E-02 & Re-188 & 4.28E-02 & 4.28E-02 \\
\hline Ac-227 & 2.09E-07 & 2.09E-07 & Ir-192m & 7.61E-07 & 7.61E-07 & Rh-102 & 3.30E-05 & 3.30E-05 \\
\hline Ac-228 & $1.92 E-08$ & 1.92E-08 & Ir-194 & $1.55 \mathrm{E}-10$ & $1.55 \mathrm{E}-10$ & $\mathrm{Rh}-103 \mathrm{~m}$ & 2.43E-03 & 2.43E-03 \\
\hline $\mathrm{Ag}-108$ & 4.96E-08 & 4.96E-08 & Ir-194m & 2.06E-03 & 2.06E-03 & Rh-106 & $1.02 E+01$ & $1.02 \mathrm{E}+01$ \\
\hline $\mathrm{Ag}-108 \mathrm{~m}$ & $9.58 \mathrm{E}-07$ & $9.58 \mathrm{E}-07$ & $\mathrm{~K}-40$ & 1.13E-07 & $1.13 \mathrm{E}-07$ & Rn-219 & 2.09E-07 & 2.09E-07 \\
\hline $\mathrm{Ag}-109 \mathrm{~m}$ & 9.54E-02 & 9.54E-02 & $\mathrm{Kr}-81$ & $4.86 \mathrm{E}-13$ & $4.86 \mathrm{E}-13$ & Rn-220 & 4.67E-05 & 4.67E-05 \\
\hline $\mathrm{Ag}-110$ & $3.15 E-04$ & $3.15 \mathrm{E}-04$ & Kr-85 & 2.09E-01 & $2.09 \mathrm{E}-01$ & Rn-222 & $3.08 \mathrm{E}-12$ & $3.08 \mathrm{E}-12$ \\
\hline $\mathrm{Ag}-110 \mathrm{~m}$ & 2.32E-02 & 2.32E-02 & La-137 & $1.55 \mathrm{E}-07$ & $1.55 \mathrm{E}-07$ & Ru-103 & 2.47E-03 & 2.47E-03 \\
\hline Am-241 & 8.48E-02 & 8.48E-02 & La-140 & 3.23E-09 & 3.23E-09 & Ru-106 & $1.00 E+01$ & $1.00 \mathrm{E}+01$ \\
\hline Am-242 & $6.19 E-04$ & 6.19E-04 & Lu-176 & 1.72E-09 & 1.72E-09 & S-35 & $1.04 \mathrm{E}+00$ & $1.04 \mathrm{E}+00$ \\
\hline$A m-242 m$ & 6.19E-04 & 6.19E-04 & Lu-177 & $3.54 \mathrm{E}-03$ & 3.54E-03 & Sb-124 & 6.92E-01 & 6.92E-01 \\
\hline Am-243 & 1.64E-04 & 1.64E-04 & Lu-177m & 1.62E-02 & 1.62E-02 & $S b-125$ & $7.41 \mathrm{E}+03$ & $7.41 \mathrm{E}+03$ \\
\hline Ar-37 & 1.63E-03 & 1.63E-03 & $M n-54$ & $6.64 \mathrm{E}+02$ & $6.64 \mathrm{E}+02$ & Sb-126 & $6.08 \mathrm{E}-06$ & $6.08 \mathrm{E}-06$ \\
\hline Ar-39 & 5.11E-03 & 5.11E-03 & Mo-93 & 2.11E-01 & 2.11E-01 & Sb-126m & 4.36E-05 & 4.36E-05 \\
\hline At-217 & 2.51E-09 & 2.51E-09 & $\mathrm{Nb}-92$ & 1.23E-06 & 1.23E-06 & Sc-46 & $1.03 E+00$ & $1.03 E+00$ \\
\hline Ba-133 & 3.41E-04 & $3.41 \mathrm{E}-04$ & $\mathrm{Nb}-93 \mathrm{~m}$ & 1.17E+02 & $1.17 E+02$ & Se-75 & $4.08 \mathrm{E}+00$ & $4.08 \mathrm{E}+00$ \\
\hline $\mathrm{Ba}-136 \mathrm{~m}$ & 3.17E-11 & $3.17 \mathrm{E}-11$ & Nb-94 & $3.71 \mathrm{E}+00$ & $3.71 E+00$ & Se-79 & $9.44 \mathrm{E}-05$ & 9.44E-05 \\
\hline Ba-137m & $4.07 \mathrm{E}+00$ & $4.07 E+00$ & Nb-95 & $2.58 \mathrm{E}+04$ & $2.58 \mathrm{E}+04$ & Si-32 & 2.57E-08 & 2.57E-08 \\
\hline $\mathrm{Ba}-140$ & 2.77E-09 & $2.77 \mathrm{E}-09$ & $\mathrm{Nb}-95 \mathrm{~m}$ & $1.38 \mathrm{E}+02$ & $1.38 E+02$ & Sm-147 & $3.12 \mathrm{E}-11$ & $3.12 \mathrm{E}-11$ \\
\hline $\mathrm{Be}-10$ & 3.38E-05 & $3.38 \mathrm{E}-05$ & $\mathrm{Nd}-147$ & $4.22 \mathrm{E}-11$ & $4.22 \mathrm{E}-11$ & Sm-151 & 7.41E-02 & 7.41E-02 \\
\hline Bi-208 & 4.27E-08 & 4.27E-08 & $\mathrm{Ni}-59$ & $5.83 E+02$ & $5.83 E+02$ & Sn-113 & $1.23 E+03$ & $1.23 E+03$ \\
\hline $\mathrm{Bi}-210 \mathrm{~m}$ & 2.39E-07 & 2.39E-07 & $\mathrm{Ni}-63$ & $7.31 \mathrm{E}+04$ & $7.31 \mathrm{E}+04$ & Sn-117m & 2.42E-06 & 2.42E-06 \\
\hline $\mathrm{Bi}-211$ & 2.09E-07 & 2.09E-07 & Np-235 & $3.53 E-10$ & $3.53 \mathrm{E}-10$ & Sn-119m & $1.24 \mathrm{E}+04$ & $1.24 \mathrm{E}+04$ \\
\hline $\mathrm{Bi}-212$ & 4.62E-05 & 4.62E-05 & Np-236 & $1.86 \mathrm{E}-12$ & 1.86E-12 & Sn-121 & $1.74 \mathrm{E}+01$ & $1.74 \mathrm{E}+01$ \\
\hline $\mathrm{Bi}-213$ & 2.51E-09 & $2.51 \mathrm{E}-09$ & Np-237 & 1.76E-06 & 1.76E-06 & Sn-121m & $2.24 \mathrm{E}+01$ & $2.24 \mathrm{E}+01$ \\
\hline Bi-214 & $3.08 \mathrm{E}-12$ & $3.08 \mathrm{E}-12$ & Np-238 & $2.78 \mathrm{E}-06$ & $2.78 \mathrm{E}-06$ & Sn-123 & $5.49 E+00$ & $5.49 \mathrm{E}+00$ \\
\hline Bk-249 & $2.05 \mathrm{E}-10$ & $2.05 \mathrm{E}-10$ & Np-239 & 1.71E-04 & 1.71E-04 & Sn-125 & $1.70 \mathrm{E}-10$ & $1.70 \mathrm{E}-10$ \\
\hline C-14 & $4.78 \mathrm{E}+01$ & $4.78 E+01$ & $\mathrm{~Np}-240 \mathrm{~m}$ & $1.55 \mathrm{E}-13$ & $1.55 \mathrm{E}-13$ & Sn-126 & 4.30E-05 & 4.30E-05 \\
\hline $\mathrm{Ca}-41$ & 8.34E-04 & 8.34E-04 & Os-185 & 2.96E-05 & 2.96E-05 & Sr-85 & 8.29E-05 & 8.29E-05 \\
\hline $\mathrm{Ca}-45$ & 1.41E-01 & 1.41E-01 & Os-191 & 1.89E-09 & 1.89E-09 & Sr-89 & 7.69E-01 & 7.69E-01 \\
\hline
\end{tabular}


TEM-10200-1

$03 / 01 / 2012$

ENGINEERING CALCULATIONS AND ANALYSIS

Page 17 of 30

Rev. 06

Baseline Radionuclide Inventory for the Remote-Handled Low-Level Waste Disposal

Title: $\quad$ Facility for Use in the Facility Performance Assessment

$\begin{array}{lllll}\text { ECAR No.: } 3940 & \text { Rev. No.: } 0 & \text { Project No.: } 31055 \quad \text { Date: } 01 / 24 / 18\end{array}$

Table 10. (continued).

\begin{tabular}{|c|c|c|c|c|c|c|c|c|}
\hline Nuclide & 20-Year & 50-Year & Nuclide & 20-Year & 50-Year & Nuclide & 20-year & 50-Year \\
\hline Cd-109 & 9.54E-02 & 9.54E-02 & Os-194 & $1.54 \mathrm{E}-10$ & $1.54 \mathrm{E}-10$ & Sr-90 & $2.02 \mathrm{E}+00$ & $2.02 E+00$ \\
\hline $\mathrm{Cd}-113 \mathrm{~m}$ & 1.41E-02 & 1.41E-02 & P-32 & 9.91E-07 & 9.91E-07 & Ta-182 & $1.52 \mathrm{E}+04$ & $1.52 \mathrm{E}+04$ \\
\hline Cd-115m & 7.78E-06 & 7.78E-06 & P-33 & 1.17E-02 & 1.17E-02 & Tb-160 & 2.68E-05 & $2.68 \mathrm{E}-05$ \\
\hline Ce-139 & 1.01E-04 & $1.01 \mathrm{E}-04$ & $\mathrm{~Pa}-231$ & $1.22 \mathrm{E}-06$ & $1.22 \mathrm{E}-06$ & Tc-99 & $3.54 \mathrm{E}-02$ & 3.54E-02 \\
\hline Ce-141 & 5.62E-04 & $5.62 \mathrm{E}-04$ & Pa-233 & 3.60E-05 & 3.60E-05 & Te-121 & 1.87E-03 & 1.87E-03 \\
\hline Ce-142 & 5.32E-08 & 5.32E-08 & $\mathrm{Pa}-234$ & 2.87E-08 & 2.87E-08 & $\mathrm{Te}-121 \mathrm{~m}$ & 1.89E-03 & 1.89E-03 \\
\hline Ce-144 & $9.39 \mathrm{E}+00$ & $9.39 \mathrm{E}+00$ & $\mathrm{~Pa}-234 \mathrm{~m}$ & 3.10E-05 & $3.10 \mathrm{E}-05$ & Te-123 & $1.23 \mathrm{E}-10$ & $1.23 \mathrm{E}-10$ \\
\hline Cf-249 & $3.29 \mathrm{E}-13$ & $3.29 \mathrm{E}-13$ & $\mathrm{~Pb}-205$ & 1.47E-07 & 1.47E-07 & $\mathrm{Te}-123 \mathrm{~m}$ & $6.65 \mathrm{E}-03$ & 6.65E-03 \\
\hline Cf-250 & $1.27 \mathrm{E}-11$ & $1.27 \mathrm{E}-11$ & Pb-209 & 2.51E-09 & 2.51E-09 & $\mathrm{Te}-125 \mathrm{~m}$ & $1.81 \mathrm{E}+03$ & $1.81 \mathrm{E}+03$ \\
\hline Cf-251 & $2.22 \mathrm{E}-16$ & $2.22 \mathrm{E}-16$ & $\mathrm{~Pb}-211$ & 2.09E-07 & 2.09E-07 & Te-127 & 1.57E-01 & 1.57E-01 \\
\hline Cf-252 & $2.48 \mathrm{E}-11$ & $2.48 \mathrm{E}-11$ & $\mathrm{~Pb}-212$ & 4.67E-05 & 4.67E-05 & Te-127m & $1.60 \mathrm{E}-01$ & 1.60E-01 \\
\hline $\mathrm{Cl}-36$ & 2.21E-02 & $2.21 \mathrm{E}-02$ & $\mathrm{~Pb}-214$ & $3.08 \mathrm{E}-12$ & $3.08 \mathrm{E}-12$ & Te-129 & $2.48 \mathrm{E}-05$ & $2.48 \mathrm{E}-05$ \\
\hline $\mathrm{Cm}-242$ & $3.52 \mathrm{E}+00$ & $3.52 E+00$ & Pd-107 & $9.18 \mathrm{E}-06$ & $9.18 \mathrm{E}-06$ & Te-129m & $3.88 \mathrm{E}-05$ & $3.88 \mathrm{E}-05$ \\
\hline Cm-243 & 4.21E-04 & 4.21E-04 & Pm-145 & $1.45 \mathrm{E}-11$ & $1.45 \mathrm{E}-11$ & Th-227 & 2.06E-07 & 2.06E-07 \\
\hline $\mathrm{Cm}-244$ & 5.99E-03 & 5.99E-03 & Pm-146 & $2.00 \mathrm{E}-08$ & $2.00 \mathrm{E}-08$ & Th-228 & 4.66E-05 & 4.66E-05 \\
\hline $\mathrm{Cm}-245$ & 8.32E-08 & 8.32E-08 & Pm-147 & $4.76 \mathrm{E}+00$ & $4.76 \mathrm{E}+00$ & Th-229 & 2.51E-09 & 2.51E-09 \\
\hline $\mathrm{Cm}-246$ & 5.03E-08 & 5.03E-08 & Pm-148 & 3.13E-06 & 3.13E-06 & Th-230 & 5.66E-09 & 5.66E-09 \\
\hline $\mathrm{Cm}-247$ & $3.34 \mathrm{E}-16$ & $3.34 \mathrm{E}-16$ & Pm-148m & $5.74 \mathrm{E}-05$ & $5.74 \mathrm{E}-05$ & Th-231 & 4.49E-07 & 4.49E-07 \\
\hline $\mathrm{Cm}-248$ & 1.06E-15 & $1.06 \mathrm{E}-15$ & Po-210 & $9.26 \mathrm{E}-02$ & $9.26 \mathrm{E}-02$ & Th-232 & $1.59 \mathrm{E}-08$ & $1.59 \mathrm{E}-08$ \\
\hline Co-58 & $5.88 \mathrm{E}+03$ & $5.88 \mathrm{E}+03$ & Po-211 & $9.24 \mathrm{E}-11$ & $9.24 \mathrm{E}-11$ & Th-234 & 3.10E-05 & 3.10E-05 \\
\hline Co-60 & $3.91 \mathrm{E}+04$ & $3.91 \mathrm{E}+04$ & Po-212 & $3.00 \mathrm{E}-05$ & $3.00 \mathrm{E}-05$ & TI-206 & 2.39E-07 & 2.39E-07 \\
\hline Cr-51 & $1.08 \mathrm{E}+01$ & $1.08 \mathrm{E}+01$ & Po-213 & $2.45 \mathrm{E}-09$ & $2.45 \mathrm{E}-09$ & TI-207 & 2.09E-07 & 2.09E-07 \\
\hline Cs-134 & $3.26 \mathrm{E}+01$ & $3.26 \mathrm{E}+01$ & Po-214 & $3.08 \mathrm{E}-12$ & $3.08 \mathrm{E}-12$ & TI-208 & 1.68E-05 & $1.68 \mathrm{E}-05$ \\
\hline Cs-135 & 4.89E-05 & 4.89E-05 & Po-215 & 2.09E-07 & 2.09E-07 & TI-209 & $2.02 \mathrm{E}-11$ & $2.02 \mathrm{E}-11$ \\
\hline Cs-136 & $2.85 \mathrm{E}-10$ & $2.85 \mathrm{E}-10$ & Po-216 & 4.67E-05 & 4.67E-05 & Tm-170 & $5.02 \mathrm{E}-12$ & $5.02 \mathrm{E}-12$ \\
\hline Cs-137 & $4.32 \mathrm{E}+00$ & $4.32 \mathrm{E}+00$ & Po-218 & $3.08 \mathrm{E}-12$ & $3.08 \mathrm{E}-12$ & $\mathrm{Tm}-171$ & $8.54 \mathrm{E}-08$ & $8.54 \mathrm{E}-08$ \\
\hline Eu-152 & $3.66 \mathrm{E}-02$ & $3.66 \mathrm{E}-02$ & Pr-143 & 8.22E-09 & 8.22E-09 & U-232 & $3.24 \mathrm{E}-05$ & $3.24 \mathrm{E}-05$ \\
\hline Eu-154 & $1.54 \mathrm{E}-01$ & $1.54 \mathrm{E}-01$ & Pr-144 & $9.51 \mathrm{E}+00$ & $9.51 \mathrm{E}+00$ & U-233 & $1.68 \mathrm{E}-05$ & $1.68 \mathrm{E}-05$ \\
\hline Eu-155 & 5.51E-02 & 5.51E-02 & Pr-144m & 1.33E-01 & 1.33E-01 & $\mathrm{U}-234$ & 2.64E-05 & 2.64E-05 \\
\hline Eu-156 & 7.51E-09 & 7.51E-09 & Pt-193 & 8.04E-05 & 8.04E-05 & U-235 & 4.49E-07 & 4.49E-07 \\
\hline $\mathrm{Fe}-55$ & $4.05 \mathrm{E}+04$ & $4.05 \mathrm{E}+04$ & Pu-236 & 1.35E-07 & $1.35 \mathrm{E}-07$ & U-236 & 4.44E-06 & 4.44E-06 \\
\hline Fe-59 & $3.57 \mathrm{E}+00$ & $3.57 \mathrm{E}+00$ & Pu-237 & 4.90E-09 & 4.90E-09 & $\mathrm{U}-237$ & 1.63E-04 & 1.63E-04 \\
\hline Fr-221 & 2.51E-09 & 2.51E-09 & Pu-238 & $6.12 \mathrm{E}-02$ & $6.12 \mathrm{E}-02$ & U-238 & $3.10 \mathrm{E}-05$ & $3.10 \mathrm{E}-05$ \\
\hline Fr-223 & $4.79 \mathrm{E}-10$ & $4.79 \mathrm{E}-10$ & Pu-239 & $6.60 \mathrm{E}-02$ & $6.60 \mathrm{E}-02$ & $\mathrm{U}-240$ & $1.55 \mathrm{E}-13$ & $1.55 \mathrm{E}-13$ \\
\hline Gd-153 & $2.55 \mathrm{E}-03$ & $2.55 \mathrm{E}-03$ & Pu-240 & 5.67E-02 & 5.67E-02 & $W-181$ & $1.39 \mathrm{E}+01$ & $1.39 \mathrm{E}+01$ \\
\hline $\mathrm{H}-3$ & $6.12 \mathrm{E}+01$ & $6.12 \mathrm{E}+01$ & Pu-241 & $6.78 \mathrm{E}+00$ & $6.78 \mathrm{E}+00$ & W-185 & $7.56 \mathrm{E}+01$ & $7.56 \mathrm{E}+01$ \\
\hline Hf-175 & $4.82 \mathrm{E}+01$ & $4.82 E+01$ & Pu-242 & 7.43E-05 & 7.43E-05 & $W-188$ & 4.24E-02 & 4.24E-02 \\
\hline Hf-178m & 4.01E-08 & $4.01 \mathrm{E}-08$ & Pu-244 & $7.74 \mathrm{E}-14$ & $7.74 \mathrm{E}-14$ & $\mathrm{Xe-131m}$ & $1.44 \mathrm{E}-11$ & $1.44 \mathrm{E}-11$ \\
\hline Hf-181 & $1.86 \mathrm{E}+01$ & $1.86 \mathrm{E}+01$ & Ra-223 & 2.09E-07 & 2.09E-07 & $Y-89 m$ & 4.95E-05 & 4.95E-05 \\
\hline Hf-182 & $1.25 \mathrm{E}-05$ & $1.25 \mathrm{E}-05$ & Ra-224 & 4.67E-05 & 4.67E-05 & Y-90 & $2.02 \mathrm{E}+00$ & $2.02 \mathrm{E}+00$ \\
\hline Ho-166m & 9.01E-09 & 9.01E-09 & Ra-225 & 2.51E-09 & 2.51E-09 & $\mathrm{Y}-91$ & $4.00 \mathrm{E}+00$ & $4.00 \mathrm{E}+00$ \\
\hline I-129 & $2.14 \mathrm{E}-06$ & $2.14 \mathrm{E}-06$ & Ra-226 & $3.08 \mathrm{E}-12$ & $3.08 \mathrm{E}-12$ & $\mathrm{Zn}-65$ & $7.01 \mathrm{E}+00$ & $7.01 \mathrm{E}+00$ \\
\hline
\end{tabular}


TEM-10200-1

$03 / 01 / 2012$

ENGINEERING CALCULATIONS AND ANALYSIS

Page 18 of 30

Rev. 06

Baseline Radionuclide Inventory for the Remote-Handled Low-Level Waste Disposal

Title: $\quad$ Facility for Use in the Facility Performance Assessment

$\begin{array}{lllll}\text { ECAR No.: } 3940 \quad \text { Rev. No.: } & 0 & \text { Project No.: } & 31055 & \text { Date: } 01 / 24 / 18\end{array}$

Table 10. (continued).

\begin{tabular}{|c|c|c|c|c|c|c|c|c|}
\hline Nuclide & 20-Year & 50-Year & Nuclide & 20-Year & 50-Year & Nuclide & 20-year & 50-Year \\
\hline In-113m & $1.23 E+03$ & $1.23 E+03$ & Ra-228 & 1.92E-08 & 1.92E-08 & Zr-93 & $6.48 E+00$ & $6.48 E+00$ \\
\hline In-114 & 7.99E-01 & 7.99E-01 & $R b-86$ & 3.93E-07 & 3.93E-07 & Zr-95 & 1.17E+04 & $1.17 E+04$ \\
\hline In-114m & 8.36E-01 & 8.36E-01 & $\mathrm{Rb}-87$ & $1.09 \mathrm{E}-07$ & 1.09E-07 & & & \\
\hline In-115m & $1.22 \mathrm{E}-10$ & $1.22 \mathrm{E}-10$ & Re-187 & 3.39E-06 & 3.39E-06 & & & \\
\hline
\end{tabular}

NRF Surface Contamination on Activated Metal Inventory for Disposal in 55-Ton Vaults

Table 11. NRF SC on AM 20-year and 50-year inventory estimates for 55-ton vaults.

\begin{tabular}{|c|c|c|c|c|c|c|c|c|}
\hline Nuclide & 20-Year & 50-Year & Nuclide & 20-Year & 50-Year & Nuclide & 20-Year & 50-Year \\
\hline Am-241 & $9.44 \mathrm{E}-04$ & $9.44 \mathrm{E}-04$ & Cs-137 & 6.92E-02 & 6.92E-02 & Pu-244 & $1.02 \mathrm{E}-13$ & $1.02 \mathrm{E}-13$ \\
\hline$A m-242 m$ & 3.33E-06 & 3.33E-06 & $\mathrm{Fe}-55$ & $1.06 \mathrm{E}+02$ & $1.06 \mathrm{E}+02$ & Ru-106 & 1.43E-01 & 1.43E-01 \\
\hline Am-243 & 8.01E-06 & 8.01E-06 & Fe-59 & $1.14 \mathrm{E}-01$ & 1.14E-01 & Sb-125 & 8.00E-01 & 8.00E-01 \\
\hline Ba-137m & 6.86E-02 & 6.86E-02 & Hf-181 & $3.25 \mathrm{E}-02$ & $3.25 E-02$ & Se-79 & 1.08E-07 & $1.08 \mathrm{E}-07$ \\
\hline$C-14$ & 8.09E-01 & 8.09E-01 & $\mathrm{I}-129$ & $2.66 \mathrm{E}-06$ & 2.66E-06 & Sn-126 & 8.36E-07 & 8.36E-07 \\
\hline Ce-144 & 1.29E-01 & 1.29E-01 & $\mathrm{Kr}-85$ & $3.50 \mathrm{E}-03$ & 3.50E-03 & Sr-90 & 6.98E-02 & $6.98 \mathrm{E}-02$ \\
\hline Cf-249 & 5.34E-13 & $5.34 \mathrm{E}-13$ & $M n-54$ & $1.29 E+01$ & $1.29 \mathrm{E}+01$ & Tc-99 & 1.43E-03 & 1.43E-03 \\
\hline Cf-251 & 1.07E-14 & $1.07 \mathrm{E}-14$ & $\mathrm{Nb}-93 \mathrm{~m}$ & $2.82 \mathrm{E}+00$ & $2.82 \mathrm{E}+00$ & $\mathrm{Te}-125 \mathrm{~m}$ & 1.86E-01 & 1.86E-01 \\
\hline $\mathrm{Cm}-242$ & 2.76E-02 & 2.76E-02 & Nb-94 & 1.15E-02 & 1.15E-02 & Th-232 & 4.75E-09 & 4.75E-09 \\
\hline $\mathrm{Cm}-243$ & 1.09E-05 & 1.09E-05 & Nb-95 & 9.06E-01 & $9.06 \mathrm{E}-01$ & U-232 & 2.81E-05 & 2.81E-05 \\
\hline Cm-244 & $6.74 \mathrm{E}-04$ & $6.74 \mathrm{E}-04$ & $\mathrm{Ni}-59$ & 3.16E-01 & 3.16E-01 & U-233 & $2.39 \mathrm{E}-07$ & 2.39E-07 \\
\hline $\mathrm{Cm}-245$ & 7.00E-08 & 7.00E-08 & $\mathrm{Ni}-63$ & $3.32 E+01$ & $3.32 E+01$ & U-234 & 4.78E-07 & 4.78E-07 \\
\hline $\mathrm{Cm}-246$ & 3.52E-08 & $3.52 \mathrm{E}-08$ & $\mathrm{~Np}-237$ & 3.35E-09 & 3.35E-09 & U-235 & 1.57E-10 & $1.57 \mathrm{E}-10$ \\
\hline $\mathrm{Cm}-247$ & $1.60 \mathrm{E}-14$ & $1.60 \mathrm{E}-14$ & $\mathrm{Pm}-147$ & 5.41E-02 & 5.41E-02 & U-236 & 5.39E-09 & 5.39E-09 \\
\hline $\mathrm{Cm}-248$ & $5.08 \mathrm{E}-14$ & $5.08 \mathrm{E}-14$ & $\mathrm{Pu}-238$ & 3.81E-04 & 3.81E-04 & U-238 & 1.40E-08 & 1.40E-08 \\
\hline Co-58 & $2.03 E+02$ & $2.03 E+02$ & Pu-239 & 7.04E-05 & 7.04E-05 & $Y-90$ & 6.98E-02 & 6.98E-02 \\
\hline Co-60 & $7.77 E+01$ & 7.77E+01 & Pu-240 & $6.22 \mathrm{E}-05$ & $6.22 \mathrm{E}-05$ & $\mathrm{Zn}-65$ & $1.23 E+00$ & $1.23 E+00$ \\
\hline Cr-51 & $8.49 E+00$ & $8.49 E+00$ & Pu-241 & 1.95E-02 & 1.95E-02 & Zr-93 & $2.25 \mathrm{E}-04$ & $2.25 \mathrm{E}-04$ \\
\hline Cs-134 & 1.60E-02 & 1.60E-02 & Pu-242 & 1.10E-06 & 1.10E-06 & Zr-95 & 3.64E-01 & 3.64E-01 \\
\hline
\end{tabular}

NRF Resin Inventory for Disposal in 55-Ton Vaults

Table 12. NRF resin 20-year and 50-year inventory estimates for 55-ton vaults.

\begin{tabular}{|c|c|c|c|c|c|c|c|c|}
\hline Nuclide & 20-Year & 50-Year & Nuclide & 20-Year & 50-Year & Nuclide & 20-Year & 50-Year \\
\hline Am-241 & $1.51 \mathrm{E}-04$ & $1.51 \mathrm{E}-04$ & $\mathrm{H}-3$ & $1.14 \mathrm{E}+00$ & $1.14 \mathrm{E}+00$ & Pu-242 & 4.91E-07 & 4.91E-07 \\
\hline Am-242 & 9.37E-07 & 9.37E-07 & Hf-175 & 4.28E-01 & 4.28E-01 & Rh-106 & 5.77E-02 & 5.77E-02 \\
\hline$A m-242 m$ & $9.42 \mathrm{E}-07$ & $9.42 \mathrm{E}-07$ & Hf-181 & $1.69 \mathrm{E}+00$ & $1.69 \mathrm{E}+00$ & Ru-106 & 5.77E-02 & 5.77E-02 \\
\hline Am-243 & $3.25 \mathrm{E}-06$ & $3.25 \mathrm{E}-06$ & I-129 & $5.52 \mathrm{E}-07$ & $5.52 \mathrm{E}-07$ & Sb-125 & $9.96 \mathrm{E}+00$ & $9.96 \mathrm{E}+00$ \\
\hline Ba-137m & $2.34 \mathrm{E}+00$ & $2.34 \mathrm{E}+00$ & In-113m & $2.74 \mathrm{E}-01$ & $2.74 \mathrm{E}-01$ & Se-79 & 1.10E-05 & 1.10E-05 \\
\hline C-14 & 2.36E-02 & 2.36E-02 & $\mathrm{Kr}-85$ & 2.32E-01 & 2.32E-01 & Sm-151 & 5.03E-03 & 5.03E-03 \\
\hline $\mathrm{Ce}-144$ & $2.75 \mathrm{E}-01$ & $2.75 \mathrm{E}-01$ & Mn-54 & $1.99 \mathrm{E}+00$ & $1.99 \mathrm{E}+00$ & Sn-113 & 2.74E-01 & 2.74E-01 \\
\hline $\mathrm{Cm}-242$ & 1.32E-06 & 1.32E-06 & Mo-99 & 9.96E-02 & 9.96E-02 & Sr-89 & 3.07E-11 & 3.07E-11 \\
\hline
\end{tabular}


TEM-10200-1

$03 / 01 / 2012$

ENGINEERING CALCULATIONS AND ANALYSIS

Page 19 of 30

Rev. 06

Baseline Radionuclide Inventory for the Remote-Handled Low-Level Waste Disposal

Title: $\quad$ Facility for Use in the Facility Performance Assessment

$\begin{array}{lllll}\text { ECAR No.: } 3940 & \text { Rev. No.: } 0 & \text { Project No.: } 31055 \quad \text { Date: } 01 / 24 / 18\end{array}$

Table 12. (continued).

\begin{tabular}{|c|c|c|c|c|c|c|c|c|}
\hline Nuclide & 20-Year & 50-Year & Nuclide & 20-Year & 50-Year & Nuclide & 20-Year & 50-Year \\
\hline $\mathrm{Cm}-243$ & $2.68 \mathrm{E}-06$ & 2.68E-06 & $\mathrm{Nb}-93 \mathrm{~m}$ & 2.57E-05 & 2.57E-05 & Sr-90 & $2.52 \mathrm{E}+00$ & $2.52 \mathrm{E}+00$ \\
\hline $\mathrm{Cm}-244$ & 1.85E-04 & $1.85 \mathrm{E}-04$ & $\mathrm{Nb}-94$ & $6.16 \mathrm{E}-10$ & $6.16 \mathrm{E}-10$ & Ta-182 & 3.86E-01 & 3.86E-01 \\
\hline Co-57 & $4.72 \mathrm{E}-02$ & $4.72 \mathrm{E}-02$ & Nb-95 & $6.26 \mathrm{E}+00$ & $6.26 \mathrm{E}+00$ & Tc-99 & 1.69E-02 & 1.69E-02 \\
\hline Co-58 & $1.75 \mathrm{E}+00$ & $1.75 \mathrm{E}+00$ & $\mathrm{Ni}-59$ & $3.39 \mathrm{E}+00$ & $3.39 \mathrm{E}+00$ & $\mathrm{Te}-125 \mathrm{~m}$ & $2.42 \mathrm{E}+00$ & $2.42 \mathrm{E}+00$ \\
\hline Co-60 & $4.93 \mathrm{E}+02$ & $4.93 E+02$ & $\mathrm{Ni}-63$ & $4.27 \mathrm{E}+02$ & $4.27 \mathrm{E}+02$ & U-234 & $8.28 \mathrm{E}-05$ & $8.28 \mathrm{E}-05$ \\
\hline Cr-51 & $4.35 \mathrm{E}+00$ & $4.35 \mathrm{E}+00$ & Np-237 & 4.49E-06 & 4.49E-06 & U-235 & 1.11E-06 & 1.11E-06 \\
\hline Cs-134 & 5.23E-01 & 5.23E-01 & Np-238 & 4.23E-09 & 4.23E-09 & U-236 & 1.27E-05 & 1.27E-05 \\
\hline Cs-137 & $2.47 \mathrm{E}+00$ & $2.47 \mathrm{E}+00$ & Np-239 & $3.25 \mathrm{E}-06$ & $3.25 \mathrm{E}-06$ & U-237 & $3.14 \mathrm{E}-07$ & $3.14 \mathrm{E}-07$ \\
\hline Eu-152 & $1.06 \mathrm{E}+00$ & $1.06 \mathrm{E}+00$ & $\mathrm{Pm}-147$ & $1.21 \mathrm{E}+00$ & $1.21 \mathrm{E}+00$ & U-238 & 5.13E-09 & 5.13E-09 \\
\hline Eu-154 & $3.39 \mathrm{E}+00$ & $3.39 \mathrm{E}+00$ & Pr-144 & $2.75 \mathrm{E}-01$ & $2.75 E-01$ & Y-90 & $2.52 E+00$ & $2.52 E+00$ \\
\hline Eu-155 & 3.39E-01 & 3.39E-01 & Pu-238 & 4.42E-02 & 4.42E-02 & Y-91 & 8.82E-10 & 8.82E-10 \\
\hline Fe-55 & $1.10 \mathrm{E}+03$ & $1.10 \mathrm{E}+03$ & Pu-239 & 3.09E-05 & 3.09E-05 & Zr-93 & 9.37E-05 & 9.37E-05 \\
\hline Fe-59 & $6.85 \mathrm{E}-01$ & $6.85 \mathrm{E}-01$ & Pu-240 & 6.31E-05 & 6.31E-05 & Zr-95 & $3.82 \mathrm{E}+00$ & $3.82 \mathrm{E}+00$ \\
\hline Gd-153 & 3.99E-02 & $3.99 \mathrm{E}-02$ & Pu-241 & 1.31E-02 & $1.31 \mathrm{E}-02$ & & & \\
\hline
\end{tabular}

NRF Activated Metal Inventory for Disposal in LCC Vaults

Table 13. NRF AM 20-year and 50-year inventory estimates for LCC vaults.

\begin{tabular}{|c|c|c|c|c|c|c|c|c|}
\hline Nuclide & $20-$ Year & 50-yeAr & Nuclide & 20 -Year & 50-Year & Nuclide & 20 -Year & 50-Year \\
\hline Ac-225 & $5.10 \mathrm{E}-08$ & $6.10 \mathrm{E}-08$ & Ir-192m & $9.90 \mathrm{E}-06$ & 1.03E-05 & Rh-102 & $6.91 \mathrm{E}-05$ & 8.91E-05 \\
\hline Ac-227 & 1.30E-06 & 1.63E-06 & Ir-194 & 3.31E-09 & 3.90E-09 & $\mathrm{Rh}-103 \mathrm{~m}$ & $6.25 \mathrm{E}-03$ & 1.97E-02 \\
\hline Ac-228 & 2.09E-07 & $2.75 \mathrm{E}-07$ & Ir-194m & 4.08E-03 & 7.76E-03 & Rh-106 & $1.96 \mathrm{E}+01$ & $2.79 E+01$ \\
\hline $\mathrm{Ag}-108$ & $9.55 \mathrm{E}-08$ & $1.31 \mathrm{E}-07$ & K-40 & 4.46E-07 & 8.31E-07 & Rn-219 & $1.31 \mathrm{E}-06$ & $1.63 \mathrm{E}-06$ \\
\hline $\mathrm{Ag}-108 \mathrm{~m}$ & 1.79E-06 & 2.41E-06 & Kr-81 & $3.36 \mathrm{E}-13$ & 2.19E-12 & Rn-220 & $1.55 \mathrm{E}-04$ & 2.38E-04 \\
\hline $\mathrm{Ag}-109 \mathrm{~m}$ & $1.94 \mathrm{E}-01$ & 2.67E-01 & Kr-85 & 4.42E-01 & 7.20E-01 & Rn-222 & $1.88 \mathrm{E}-11$ & $7.50 \mathrm{E}-11$ \\
\hline $\mathrm{Ag}-110$ & 5.81E-04 & 8.68E-04 & La-137 & 2.22E-06 & 2.22E-06 & Ru-103 & $6.28 \mathrm{E}-03$ & 1.98E-02 \\
\hline $\mathrm{Ag}-110 \mathrm{~m}$ & 4.27E-02 & 6.38E-02 & La-140 & 8.74E-09 & 3.01E-08 & Ru-106 & $1.91 \mathrm{E}+01$ & $2.72 E+01$ \\
\hline Am-241 & 1.57E-01 & 2.19E-01 & Lu-176 & 4.02E-09 & 9.12E-09 & S-35 & $2.66 \mathrm{E}+00$ & $4.47 E+00$ \\
\hline Am-242 & 1.18E-03 & 2.07E-03 & Lu-177 & 7.02E-03 & 1.17E-02 & Sb-124 & $1.50 E+00$ & $3.71 E+00$ \\
\hline$A m-242 m$ & 1.15E-03 & 1.84E-03 & Lu-177m & $3.20 \mathrm{E}-02$ & 5.36E-02 & Sb-125 & $1.53 E+04$ & $2.71 E+04$ \\
\hline Am-243 & 2.96E-04 & 5.92E-04 & $M n-54$ & $1.31 \mathrm{E}+03$ & $2.07 E+03$ & Sb-126 & 1.23E-05 & 2.03E-05 \\
\hline Ar-37 & 4.64E-03 & 1.05E-02 & Mo-93 & $2.61 \mathrm{E}-01$ & $6.87 \mathrm{E}-01$ & Sb-126m & 8.80E-05 & $1.45 \mathrm{E}-04$ \\
\hline Ar-39 & 2.67E-02 & 4.33E-02 & $\mathrm{Nb}-92$ & 3.20E-06 & 4.65E-06 & Sc-46 & $2.87 E+00$ & $4.08 \mathrm{E}+00$ \\
\hline At-217 & 5.10E-08 & 6.10E-08 & $\mathrm{Nb}-93 \mathrm{~m}$ & $3.77 E+02$ & $4.74 \mathrm{E}+02$ & Se-75 & $8.59 E+00$ & $1.33 E+01$ \\
\hline Ba-133 & 8.91E-04 & 1.47E-03 & $\mathrm{Nb}-94$ & $8.31 \mathrm{E}+00$ & $1.81 \mathrm{E}+01$ & Se-79 & $2.27 \mathrm{E}-04$ & 4.58E-04 \\
\hline Ba-136m & $7.59 \mathrm{E}-11$ & $3.04 \mathrm{E}-10$ & $\mathrm{Nb}-95$ & $5.32 \mathrm{E}+04$ & $8.49 \mathrm{E}+04$ & $\mathrm{Si}-32$ & $3.81 \mathrm{E}-08$ & $1.04 \mathrm{E}-07$ \\
\hline Ba-137m & $8.40 \mathrm{E}+00$ & $1.37 E+01$ & $\mathrm{Nb}-95 \mathrm{~m}$ & $2.85 E+02$ & $4.53 E+02$ & Sm-147 & $1.07 \mathrm{E}-10$ & $3.97 \mathrm{E}-10$ \\
\hline Ba-140 & 6.99E-09 & $2.55 \mathrm{E}-08$ & Nd-147 & $9.62 \mathrm{E}-11$ & $3.78 \mathrm{E}-10$ & Sm-151 & $1.58 \mathrm{E}-01$ & $2.53 \mathrm{E}-01$ \\
\hline $\mathrm{Be}-10$ & $1.09 \mathrm{E}-04$ & $1.33 \mathrm{E}-04$ & $\mathrm{Ni}-59$ & $9.30 \mathrm{E}+02$ & $1.98 \mathrm{E}+03$ & Sn-113 & $2.46 \mathrm{E}+03$ & $3.76 \mathrm{E}+03$ \\
\hline $\mathrm{Bi}-208$ & $2.21 \mathrm{E}-07$ & $2.72 \mathrm{E}-07$ & $\mathrm{Ni}-63$ & $1.13 E+05$ & $2.45 E+05$ & Sn-117m & $5.45 \mathrm{E}-06$ & 1.84E-05 \\
\hline $\mathrm{Bi}-210 \mathrm{~m}$ & $5.26 \mathrm{E}-07$ & $8.45 \mathrm{E}-07$ & Np-235 & $1.24 \mathrm{E}-09$ & 2.11E-09 & Sn-119m & $2.44 E+04$ & $3.77 E+04$ \\
\hline $\mathrm{Bi}-211$ & 1.31E-06 & 1.63E-06 & Np-236 & $9.06 \mathrm{E}-12$ & 1.05E-11 & Sn-121 & $4.02 E+01$ & $8.78 E+01$ \\
\hline
\end{tabular}


TEM-10200-1

$03 / 01 / 2012$

ENGINEERING CALCULATIONS AND ANALYSIS

Page 20 of 30

Rev. 06

Baseline Radionuclide Inventory for the Remote-Handled Low-Level Waste Disposal

Title: $\quad$ Facility for Use in the Facility Performance Assessment

$\begin{array}{lllll}\text { ECAR No.: } 3940 & \text { Rev. No.: } 0 & \text { Project No.: } 31055 \quad \text { Date: } 01 / 24 / 18\end{array}$

Table 13. (continued).

\begin{tabular}{|c|c|c|c|c|c|c|c|c|}
\hline Nuclide & 20-Year & 50-yeAr & Nuclide & 20-Year & 50-Year & Nuclide & 20-Year & 50-Year \\
\hline $\mathrm{Bi}-212$ & $1.54 \mathrm{E}-04$ & 2.37E-04 & Np-237 & 4.49E-06 & 6.62E-06 & Sn-121m & $5.19 \mathrm{E}+01$ & $1.13 E+02$ \\
\hline $\mathrm{Bi}-213$ & 5.10E-08 & $6.10 \mathrm{E}-08$ & Np-238 & 5.32E-06 & $9.27 \mathrm{E}-06$ & Sn-123 & $1.13 \mathrm{E}+01$ & $1.80 \mathrm{E}+01$ \\
\hline $\mathrm{Bi}-214$ & $1.88 \mathrm{E}-11$ & $7.50 \mathrm{E}-11$ & Np-239 & 3.37E-04 & 7.71E-04 & Sn-125 & $4.08 \mathrm{E}-10$ & 1.60E-09 \\
\hline Bk-249 & $4.45 \mathrm{E}-10$ & 1.79E-09 & Np-240m & $1.11 \mathrm{E}-13$ & $7.22 \mathrm{E}-13$ & Sn-126 & 8.57E-05 & 1.36E-04 \\
\hline C-14 & $1.12 \mathrm{E}+02$ & $2.54 \mathrm{E}+02$ & Os-185 & 4.78E-05 & $1.55 \mathrm{E}-04$ & Sr-85 & 1.69E-04 & 4.65E-04 \\
\hline Ca-41 & 3.32E-03 & $6.18 \mathrm{E}-03$ & Os-191 & 6.20E-09 & $2.50 \mathrm{E}-08$ & Sr-89 & $2.10 \mathrm{E}+00$ & $3.57 \mathrm{E}+00$ \\
\hline Ca-45 & 3.83E-01 & $6.02 \mathrm{E}-01$ & Os-194 & $3.28 \mathrm{E}-09$ & 3.88E-09 & Sr-90 & $4.36 \mathrm{E}+00$ & $7.29 \mathrm{E}+00$ \\
\hline Cd-109 & 1.94E-01 & 2.67E-01 & P-32 & $2.65 \mathrm{E}-06$ & 5.04E-06 & Ta-180 & $5.18 \mathrm{E}-12$ & $2.58 \mathrm{E}-11$ \\
\hline $\mathrm{Cd}-113 \mathrm{~m}$ & $2.88 \mathrm{E}-02$ & 4.18E-02 & P-33 & $2.78 \mathrm{E}-02$ & 4.65E-02 & Ta-182 & $3.09 \mathrm{E}+04$ & $4.14 \mathrm{E}+04$ \\
\hline$C d-115 m$ & 3.84E-05 & $1.04 \mathrm{E}-04$ & Pa-231 & 5.46E-06 & 7.52E-06 & Tb-160 & $5.75 \mathrm{E}-05$ & 2.09E-04 \\
\hline Ce-139 & $2.29 \mathrm{E}-04$ & 4.07E-04 & $\mathrm{Pa}-233$ & 8.66E-05 & $1.56 \mathrm{E}-04$ & Tc-99 & 3.73E-02 & 8.72E-02 \\
\hline Ce-141 & 1.46E-03 & 4.36E-03 & $\mathrm{Pa}-234$ & 1.10E-07 & 2.97E-07 & Te-121 & 3.97E-03 & 5.97E-03 \\
\hline Ce-142 & 5.32E-07 & 5.33E-07 & $\mathrm{Pa}-234 \mathrm{~m}$ & $1.04 \mathrm{E}-04$ & $2.53 E-04$ & Te-121m & 4.00E-03 & $6.00 \mathrm{E}-03$ \\
\hline Ce-144 & $1.84 \mathrm{E}+01$ & $2.62 \mathrm{E}+01$ & Pb-205 & $2.28 \mathrm{E}-07$ & 4.47E-07 & Te-123 & 1.11E-09 & 4.63E-09 \\
\hline Cf-249 & $2.49 \mathrm{E}-13$ & $1.05 \mathrm{E}-12$ & Pb-209 & $5.10 \mathrm{E}-08$ & $6.10 \mathrm{E}-08$ & Te-123m & $8.52 \mathrm{E}-03$ & 2.12E-02 \\
\hline Cf-250 & $3.25 \mathrm{E}-11$ & $1.31 \mathrm{E}-10$ & $\mathrm{~Pb}-211$ & $1.31 \mathrm{E}-06$ & 1.63E-06 & $\mathrm{Te}-125 \mathrm{~m}$ & $3.73 E+03$ & $6.61 \mathrm{E}+03$ \\
\hline Cf-251 & $7.74 \mathrm{E}-16$ & $3.04 \mathrm{E}-15$ & $\mathrm{~Pb}-212$ & $1.55 \mathrm{E}-04$ & 2.38E-04 & Te-127 & $3.22 \mathrm{E}-01$ & 4.71E-01 \\
\hline Cf-252 & $7.41 \mathrm{E}-11$ & $2.99 \mathrm{E}-10$ & $\mathrm{~Pb}-214$ & $1.88 \mathrm{E}-11$ & $7.50 \mathrm{E}-11$ & Te-127m & $3.29 \mathrm{E}-01$ & 4.81E-01 \\
\hline $\mathrm{Cl}-36$ & $9.24 \mathrm{E}-02$ & $1.30 \mathrm{E}-01$ & Pd-107 & $1.85 \mathrm{E}-05$ & $3.05 \mathrm{E}-05$ & Te-129 & $6.82 \mathrm{E}-05$ & $2.10 \mathrm{E}-04$ \\
\hline $\mathrm{Cm}-242$ & $6.44 \mathrm{E}+00$ & $8.00 \mathrm{E}+00$ & Pm-145 & $3.87 \mathrm{E}-11$ & $1.44 \mathrm{E}-10$ & $\mathrm{Te}-129 \mathrm{~m}$ & 1.07E-04 & 3.28E-04 \\
\hline $\mathrm{Cm}-243$ & $7.26 \mathrm{E}-04$ & 1.13E-03 & Pm-146 & $4.00 \mathrm{E}-08$ & $6.36 \mathrm{E}-08$ & Th-227 & $1.29 \mathrm{E}-06$ & $1.61 \mathrm{E}-06$ \\
\hline $\mathrm{Cm}-244$ & 1.12E-02 & 2.87E-02 & Pm-147 & $9.63 \mathrm{E}+00$ & $1.46 \mathrm{E}+01$ & Th-228 & $1.54 \mathrm{E}-04$ & 2.37E-04 \\
\hline $\mathrm{Cm}-245$ & $1.55 \mathrm{E}-07$ & $6.21 \mathrm{E}-07$ & Pm-148 & $6.05 \mathrm{E}-06$ & $2.03 E-05$ & Th-229 & $5.10 \mathrm{E}-08$ & $6.10 \mathrm{E}-08$ \\
\hline $\mathrm{Cm}-246$ & $1.22 \mathrm{E}-07$ & 4.87E-07 & Pm-148m & 1.10E-04 & 3.77E-04 & Th-230 & 4.24E-08 & $6.19 \mathrm{E}-08$ \\
\hline $\mathrm{Cm}-247$ & 1.17E-15 & $4.58 \mathrm{E}-15$ & Po-210 & 1.91E-01 & $2.71 \mathrm{E}-01$ & Th-231 & $2.53 \mathrm{E}-06$ & 7.57E-06 \\
\hline $\mathrm{Cm}-248$ & $3.69 \mathrm{E}-15$ & $1.45 \mathrm{E}-14$ & Po-211 & 2.93E-09 & 2.93E-09 & Th-232 & 1.12E-07 & $1.39 \mathrm{E}-07$ \\
\hline Co-58 & $1.19 \mathrm{E}+04$ & $1.82 \mathrm{E}+04$ & Po-212 & 9.91E-05 & $1.52 \mathrm{E}-04$ & Th-234 & 1.04E-04 & $2.53 \mathrm{E}-04$ \\
\hline Co-60 & $6.17 \mathrm{E}+04$ & $1.45 \mathrm{E}+05$ & Po-213 & 4.98E-08 & 5.96E-08 & TI-206 & $5.26 \mathrm{E}-07$ & 8.45E-07 \\
\hline Cr-51 & $1.47 \mathrm{E}+01$ & $5.87 \mathrm{E}+01$ & Po-214 & $1.88 \mathrm{E}-11$ & $7.50 \mathrm{E}-11$ & TI-207 & $1.30 \mathrm{E}-06$ & 1.63E-06 \\
\hline Cs-134 & $7.00 \mathrm{E}+01$ & $1.28 \mathrm{E}+02$ & Po-215 & $1.31 \mathrm{E}-06$ & 1.63E-06 & TI-208 & $5.58 \mathrm{E}-05$ & 8.56E-05 \\
\hline Cs-135 & 1.06E-04 & 1.92E-04 & Po-216 & $1.55 \mathrm{E}-04$ & 2.38E-04 & TI-209 & $6.66 \mathrm{E}-11$ & $2.68 \mathrm{E}-10$ \\
\hline Cs-136 & $6.84 \mathrm{E}-10$ & $2.74 \mathrm{E}-09$ & Po-218 & $1.88 \mathrm{E}-11$ & $7.50 \mathrm{E}-11$ & Tm-170 & $1.04 \mathrm{E}-11$ & 4.13E-11 \\
\hline Cs-137 & $8.90 \mathrm{E}+00$ & $1.45 \mathrm{E}+01$ & Pr-143 & $2.38 \mathrm{E}-08$ & 7.82E-08 & $\mathrm{Tm}-171$ & $1.69 \mathrm{E}-07$ & $2.57 \mathrm{E}-07$ \\
\hline Eu-152 & $9.17 \mathrm{E}-02$ & $1.51 \mathrm{E}-01$ & Pr-144 & $1.89 \mathrm{E}+01$ & $2.68 \mathrm{E}+01$ & $\mathrm{U}-232$ & $6.64 \mathrm{E}-05$ & $9.16 \mathrm{E}-05$ \\
\hline Eu-154 & $3.05 \mathrm{E}-01$ & 5.27E-01 & Pr-144m & $2.64 \mathrm{E}-01$ & $3.75 \mathrm{E}-01$ & U-233 & $6.23 \mathrm{E}-05$ & $9.66 \mathrm{E}-05$ \\
\hline Eu-155 & 1.15E-01 & $1.90 \mathrm{E}-01$ & Pt-193 & $5.84 \mathrm{E}-04$ & $8.20 \mathrm{E}-04$ & $\mathrm{U}-234$ & $9.36 \mathrm{E}-05$ & $2.32 \mathrm{E}-04$ \\
\hline Eu-156 & $1.84 \mathrm{E}-08$ & 7.36E-08 & Pu-236 & $3.80 \mathrm{E}-07$ & 5.64E-07 & U-235 & $2.53 \mathrm{E}-06$ & 7.57E-06 \\
\hline $\mathrm{Fe}-55$ & $7.60 \mathrm{E}+04$ & $1.56 \mathrm{E}+05$ & Pu-237 & 2.97E-08 & 4.17E-08 & $\mathrm{U}-236$ & 1.03E-05 & 1.80E-05 \\
\hline Fe-59 & $5.00 \mathrm{E}+00$ & $1.42 \mathrm{E}+01$ & Pu-238 & 1.15E-01 & $1.85 \mathrm{E}-01$ & $\mathrm{U}-237$ & 3.08E-04 & $4.75 \mathrm{E}-04$ \\
\hline Fr-221 & $5.10 \mathrm{E}-08$ & $6.10 \mathrm{E}-08$ & Pu-239 & 1.47E-01 & 2.36E-01 & U-238 & $1.04 \mathrm{E}-04$ & $2.53 \mathrm{E}-04$ \\
\hline Fr-223 & $1.52 \mathrm{E}-08$ & $1.52 \mathrm{E}-08$ & Pu-240 & 1.15E-01 & 1.81E-01 & $\mathrm{U}-240$ & $1.11 \mathrm{E}-13$ & $7.22 \mathrm{E}-13$ \\
\hline Gd-153 & $5.26 \mathrm{E}-03$ & $7.68 \mathrm{E}-03$ & Pu-241 & $1.27 \mathrm{E}+01$ & $1.90 \mathrm{E}+01$ & W-181 & $2.90 \mathrm{E}+01$ & $4.62 \mathrm{E}+01$ \\
\hline
\end{tabular}


TEM-10200-1

$03 / 01 / 2012$

ENGINEERING CALCULATIONS AND ANALYSIS

Page 21 of 30

Rev. 06

Baseline Radionuclide Inventory for the Remote-Handled Low-Level Waste Disposal

Title: $\quad$ Facility for Use in the Facility Performance Assessment

$\begin{array}{lllll}\text { ECAR No.: } 3940 \quad \text { Rev. No.: } & 0 & \text { Project No.: } 31055 \quad \text { Date: } 01 / 24 / 18\end{array}$

Table 13. (continued).

\begin{tabular}{|c|c|c|c|c|c|c|c|c|}
\hline Nuclide & 20-Year & 50-yeAr & Nuclide & 20-Year & 50-Year & Nuclide & 20-Year & 50-Year \\
\hline $\mathrm{H}-3$ & $1.47 \mathrm{E}+02$ & $3.17 \mathrm{E}+02$ & Pu-242 & 1.39E-04 & $2.38 \mathrm{E}-04$ & W-185 & $1.56 \mathrm{E}+02$ & $2.44 \mathrm{E}+02$ \\
\hline Hf-175 & $1.04 \mathrm{E}+02$ & $1.52 \mathrm{E}+02$ & Pu-244 & $4.49 \mathrm{E}-14$ & $1.63 E-13$ & W-188 & 8.31E-02 & 1.45E-01 \\
\hline Hf-181 & $4.35 \mathrm{E}+01$ & 1.07E+02 & Ra-223 & 1.31E-06 & 1.63E-06 & $X e-131 m$ & $3.32 \mathrm{E}-11$ & $1.25 \mathrm{E}-10$ \\
\hline Hf-182 & $2.89 \mathrm{E}-05$ & 6.82E-05 & Ra-224 & $1.55 \mathrm{E}-04$ & $2.38 \mathrm{E}-04$ & Y-89m & 1.54E-04 & 2.80E-04 \\
\hline Ho-166m & 1.96E-08 & 4.58E-08 & Ra-225 & $5.10 \mathrm{E}-08$ & 6.10E-08 & Y-90 & $4.36 E+00$ & $7.29 E+00$ \\
\hline I-129 & 5.87E-06 & 1.10E-05 & Ra-226 & $1.88 \mathrm{E}-11$ & $7.50 \mathrm{E}-11$ & Y-91 & $1.08 \mathrm{E}+01$ & $1.85 \mathrm{E}+01$ \\
\hline In-113m & $2.46 \mathrm{E}+03$ & $3.76 \mathrm{E}+03$ & Ra-228 & 2.09E-07 & $2.75 E-07$ & $\mathrm{Zn}-65$ & $1.06 \mathrm{E}+01$ & $2.03 E+01$ \\
\hline In-114 & $1.48 \mathrm{E}+00$ & $3.35 \mathrm{E}+00$ & $\mathrm{Rb}-86$ & 1.01E-06 & 1.81E-06 & Zr-93 & $1.56 \mathrm{E}+01$ & $3.64 \mathrm{E}+01$ \\
\hline In-114m & $1.54 \mathrm{E}+00$ & $3.51 \mathrm{E}+00$ & Rb-87 & 1.17E-06 & 1.17E-06 & Zr-95 & $2.41 \mathrm{E}+04$ & $3.84 \mathrm{E}+04$ \\
\hline In-115m & $2.76 \mathrm{E}-10$ & 1.00E-09 & $\operatorname{Re}-187$ & 8.07E-06 & 1.77E-05 & & & \\
\hline Ir-192 & $3.53 \mathrm{E}-02$ & 7.41E-02 & $\operatorname{Re}-188$ & 8.39E-02 & 1.47E-01 & & & \\
\hline
\end{tabular}

NRF Surface Contamination on Activated Metal Inventory for Disposal in LCC Vaults

Table 14. NRF SC on AM 20-year and 50-year inventory estimates for LCC vaults.

\begin{tabular}{|c|c|c|c|c|c|c|c|c|}
\hline Nuclide & 20-Year & 50-Year & Nuclide & 20-Year & 50-Year & Nuclide & 20-Year & 50-Year \\
\hline Am-241 & $6.59 \mathrm{E}-03$ & 2.98E-02 & Cs-137 & 3.39E-01 & $1.02 E+00$ & Pu-244 & $2.87 \mathrm{E}-13$ & $9.42 \mathrm{E}-13$ \\
\hline$A m-242 m$ & 4.34E-05 & 2.59E-04 & $\mathrm{Fe}-55$ & $1.20 \mathrm{E}+03$ & $4.82 E+03$ & Ru-106 & 6.38E-01 & $9.65 \mathrm{E}-01$ \\
\hline $\mathrm{Am}-243$ & $5.60 \mathrm{E}-05$ & $2.26 \mathrm{E}-04$ & $\mathrm{Fe}-59$ & 5.27E-01 & $1.65 \mathrm{E}+00$ & Sb-125 & $5.96 \mathrm{E}+00$ & $2.14 \mathrm{E}+01$ \\
\hline Ba-137m & 3.31E-01 & $9.84 \mathrm{E}-01$ & Hf-181 & $1.38 \mathrm{E}-01$ & 4.59E-01 & Se-79 & 1.13E-06 & 4.24E-06 \\
\hline C-14 & $6.98 \mathrm{E}+00$ & $3.01 \mathrm{E}+01$ & I-129 & 1.94E-05 & 6.04E-05 & Sn-126 & 4.51E-06 & 1.56E-05 \\
\hline $\mathrm{Ce}-144$ & $5.64 \mathrm{E}-01$ & 8.84E-01 & Kr-85 & 1.66E-02 & $4.26 \mathrm{E}-02$ & Sr-90 & 3.07E-01 & 8.30E-01 \\
\hline Cf-249 & $2.60 \mathrm{E}-12$ & $8.10 \mathrm{E}-12$ & Mn-54 & $1.39 \mathrm{E}+02$ & $3.48 \mathrm{E}+02$ & Tc-99 & 8.29E-03 & 4.06E-02 \\
\hline Cf-251 & $9.74 \mathrm{E}-14$ & $3.04 \mathrm{E}-13$ & $\mathrm{Nb}-93 \mathrm{~m}$ & $7.32 \mathrm{E}+01$ & $1.00 \mathrm{E}+02$ & Te-125m & $1.43 \mathrm{E}+00$ & $5.23 \mathrm{E}+00$ \\
\hline $\mathrm{Cm}-242$ & $2.22 \mathrm{E}-01$ & $8.75 \mathrm{E}-01$ & $\mathrm{Nb}-94$ & 1.46E-01 & $6.08 \mathrm{E}-01$ & Th-232 & 1.15E-07 & $1.50 \mathrm{E}-07$ \\
\hline Cm-243 & $8.12 \mathrm{E}-05$ & 2.95E-04 & $\mathrm{Nb}-95$ & $3.85 \mathrm{E}+00$ & $1.14 \mathrm{E}+01$ & U-232 & 1.04E-04 & 2.03E-04 \\
\hline $\mathrm{Cm}-244$ & $4.21 \mathrm{E}-03$ & 1.57E-02 & $\mathrm{Ni}-59$ & $3.19 \mathrm{E}+00$ & $1.38 \mathrm{E}+01$ & U-233 & $9.95 \mathrm{E}-07$ & $3.13 \mathrm{E}-06$ \\
\hline Cm-245 & 2.19E-07 & 6.92E-07 & $\mathrm{Ni}-63$ & $3.21 \mathrm{E}+02$ & $1.41 \mathrm{E}+03$ & U-234 & 1.59E-06 & 5.91E-06 \\
\hline $\mathrm{Cm}-246$ & $1.45 \mathrm{E}-07$ & 4.69E-07 & Np-237 & 6.89E-08 & 1.80E-07 & U-235 & $2.18 \mathrm{E}-10$ & 1.74E-09 \\
\hline $\mathrm{Cm}-247$ & $1.46 \mathrm{E}-13$ & $4.56 \mathrm{E}-13$ & Pm-147 & 2.11E-01 & 3.61E-01 & U-236 & 8.30E-09 & 1.86E-08 \\
\hline $\mathrm{Cm}-248$ & $4.63 \mathrm{E}-13$ & $1.45 \mathrm{E}-12$ & Pu-238 & $5.82 \mathrm{E}-03$ & 3.62E-02 & U-238 & 2.92E-08 & 1.13E-07 \\
\hline Co-58 & $1.00 \mathrm{E}+03$ & $2.62 E+03$ & Pu-239 & $3.78 \mathrm{E}-04$ & $9.41 \mathrm{E}-04$ & $Y-90$ & 3.07E-01 & $8.30 \mathrm{E}-01$ \\
\hline Co-60 & $6.38 \mathrm{E}+02$ & $2.61 \mathrm{E}+03$ & Pu-240 & $3.13 E-04$ & 1.18E-03 & $\mathrm{Zn}-65$ & $8.68 E+00$ & $2.70 \mathrm{E}+01$ \\
\hline Cr-51 & $7.36 \mathrm{E}+00$ & $1.08 \mathrm{E}+01$ & Pu-241 & $1.88 \mathrm{E}-01$ & $9.81 \mathrm{E}-01$ & Zr-93 & $1.70 \mathrm{E}-03$ & 7.95E-03 \\
\hline Cs-134 & 1.02E-01 & $3.53 \mathrm{E}-01$ & $\mathrm{Pu}-242$ & 9.67E-06 & 4.79E-05 & Zr-95 & $1.61 \mathrm{E}+00$ & $5.05 E+00$ \\
\hline
\end{tabular}


TEM-10200-1

$03 / 01 / 2012$

ENGINEERING CALCULATIONS AND ANALYSIS

Page 22 of 30

Rev. 06

Baseline Radionuclide Inventory for the Remote-Handled Low-Level Waste Disposal

Title: $\quad$ Facility for Use in the Facility Performance Assessment

$\begin{array}{lllll}\text { ECAR No.: } 3940 & \text { Rev. No.: } 0 & \text { Project No.: } 31055 \quad \text { Date: } 01 / 24 / 18\end{array}$

\section{NRF Resin Inventory for Disposal in LCC Vaults}

Table 15. NRF resin 20-year and 50-year inventory estimates for LCC vaults.

\begin{tabular}{|c|c|c|c|c|c|c|c|c|}
\hline Nuclide & 20-Year & 50-Year & Nuclide & 20-Year & 50-Year & Nuclide & 20-Year & 50-Year \\
\hline Am-241 & $3.46 \mathrm{E}-04$ & 1.09E-03 & $\mathrm{H}-3$ & $2.61 \mathrm{E}+00$ & $8.24 \mathrm{E}+00$ & Pu-242 & 1.12E-06 & $3.55 \mathrm{E}-06$ \\
\hline Am-242 & $2.15 \mathrm{E}-06$ & $6.77 \mathrm{E}-06$ & Hf-175 & $9.82 \mathrm{E}-01$ & $3.10 \mathrm{E}+00$ & Rh-106 & 1.32E-01 & 4.17E-01 \\
\hline$A m-242 m$ & 2.16E-06 & 6.81E-06 & Hf-181 & $3.87 \mathrm{E}+00$ & $1.22 \mathrm{E}+01$ & Ru-106 & 1.32E-01 & 4.17E-01 \\
\hline Am-243 & 7.45E-06 & 2.35E-05 & I-129 & 1.27E-06 & 3.99E-06 & Sb-125 & $2.28 \mathrm{E}+01$ & $7.20 E+01$ \\
\hline Ba-137m & $5.36 \mathrm{E}+00$ & $1.69 E+01$ & In-113m & $6.28 \mathrm{E}-01$ & $1.98 \mathrm{E}+00$ & Se-79 & $2.53 \mathrm{E}-05$ & 7.99E-05 \\
\hline$C-14$ & $5.40 \mathrm{E}-02$ & 1.70E-01 & $\mathrm{Kr}-85$ & $5.32 \mathrm{E}-01$ & $1.68 \mathrm{E}+00$ & Sm-151 & 1.15E-02 & 3.64E-02 \\
\hline Ce-144 & 6.30E-01 & $1.99 \mathrm{E}+00$ & $M n-54$ & $4.57 \mathrm{E}+00$ & $1.44 \mathrm{E}+01$ & Sn-113 & $6.28 \mathrm{E}-01$ & $1.98 \mathrm{E}+00$ \\
\hline $\mathrm{Cm}-242$ & 3.03E-06 & $9.55 \mathrm{E}-06$ & Mo-99 & $2.28 \mathrm{E}-01$ & 7.20E-01 & Sr-89 & 7.03E-11 & $2.22 \mathrm{E}-10$ \\
\hline $\mathrm{Cm}-243$ & $6.14 \mathrm{E}-06$ & 1.94E-05 & $\mathrm{Nb}-93 \mathrm{~m}$ & 5.90E-05 & 1.86E-04 & Sr-90 & $5.78 \mathrm{E}+00$ & $1.82 E+01$ \\
\hline $\mathrm{Cm}-244$ & 4.24E-04 & $1.34 \mathrm{E}-03$ & $\mathrm{Nb}-94$ & 1.41E-09 & 4.46E-09 & Ta-182 & 8.84E-01 & $2.79 E+00$ \\
\hline Co-57 & $1.08 \mathrm{E}-01$ & 3.41E-01 & Nb-95 & $1.44 \mathrm{E}+01$ & $4.53 E+01$ & Tc-99 & 3.87E-02 & $1.22 \mathrm{E}-01$ \\
\hline Co-58 & $4.00 \mathrm{E}+00$ & $1.26 E+01$ & $\mathrm{Ni}-59$ & $7.76 \mathrm{E}+00$ & $2.45 E+01$ & $\mathrm{Te}-125 \mathrm{~m}$ & $5.55 \mathrm{E}+00$ & $1.75 E+01$ \\
\hline Co-60 & $1.13 \mathrm{E}+03$ & $3.56 \mathrm{E}+03$ & $\mathrm{Ni}-63$ & $9.80 \mathrm{E}+02$ & $3.09 \mathrm{E}+03$ & U-234 & $1.90 \mathrm{E}-04$ & 5.99E-04 \\
\hline Cr-51 & $9.98 \mathrm{E}+00$ & $3.15 \mathrm{E}+01$ & Np-237 & 1.03E-05 & $3.24 \mathrm{E}-05$ & U-235 & $2.54 \mathrm{E}-06$ & 8.02E-06 \\
\hline Cs-134 & $1.20 \mathrm{E}+00$ & $3.78 \mathrm{E}+00$ & Np-238 & $9.71 \mathrm{E}-09$ & 3.06E-08 & $\mathrm{U}-236$ & 2.90E-05 & $9.16 \mathrm{E}-05$ \\
\hline Cs-137 & $5.66 \mathrm{E}+00$ & $1.79 \mathrm{E}+01$ & Np-239 & $7.45 \mathrm{E}-06$ & 2.35E-05 & U-237 & 7.20E-07 & $2.27 \mathrm{E}-06$ \\
\hline Eu-152 & $2.42 E+00$ & $7.63 \mathrm{E}+00$ & Pm-147 & $2.78 E+00$ & $8.77 \mathrm{E}+00$ & U-238 & 1.18E-08 & $3.71 \mathrm{E}-08$ \\
\hline Eu-154 & $7.76 \mathrm{E}+00$ & $2.45 \mathrm{E}+01$ & Pr-144 & $6.30 \mathrm{E}-01$ & $1.99 \mathrm{E}+00$ & Y-90 & $5.78 \mathrm{E}+00$ & $1.82 \mathrm{E}+01$ \\
\hline Eu-155 & 7.76E-01 & $2.45 \mathrm{E}+00$ & Pu-238 & $1.01 \mathrm{E}-01$ & 3.19E-01 & Y-91 & 2.02E-09 & 6.38E-09 \\
\hline $\mathrm{Fe}-55$ & $2.53 E+03$ & $7.99 \mathrm{E}+03$ & Pu-239 & 7.07E-05 & 2.23E-04 & Zr-93 & $2.15 \mathrm{E}-04$ & 6.77E-04 \\
\hline $\mathrm{Fe}-59$ & $1.57 \mathrm{E}+00$ & $4.96 \mathrm{E}+00$ & Pu-240 & $1.45 \mathrm{E}-04$ & 4.56E-04 & Zr-95 & $8.76 \mathrm{E}+00$ & $2.76 \mathrm{E}+01$ \\
\hline Gd-153 & $9.14 \mathrm{E}-02$ & 2.88E-01 & Pu-241 & 2.99E-02 & $9.45 \mathrm{E}-02$ & & & \\
\hline
\end{tabular}

RH-LLW Disposal Facility Total 20-Year and 50 -Year Inventory by Waste Type

Table 16 . Total 20 -year and 50 -year inventory by waste type.

\begin{tabular}{|c|c|c|c|c|c|c|c|c|}
\hline \multirow[b]{2}{*}{ Nuclide } & \multicolumn{3}{|c|}{ 20-Year Inventory } & \multirow[b]{2}{*}{$\begin{array}{c}20-Y e a r \\
\text { Total }\end{array}$} & \multicolumn{4}{|c|}{ 50-Year Inventory } \\
\hline & AM & sc & Resin & & AM & sc & Resin & $\begin{array}{c}\text { 50-Year } \\
\text { Total }\end{array}$ \\
\hline Ac-225 & 5.35E-08 & & & 5.35E-08 & $6.35 \mathrm{E}-08$ & & & 6.35E-08 \\
\hline Ac-227 & $1.51 \mathrm{E}-06$ & 4.25E-06 & & $5.76 \mathrm{E}-06$ & $1.84 \mathrm{E}-06$ & $1.05 \mathrm{E}-05$ & & $1.24 \mathrm{E}-05$ \\
\hline Ac-228 & $2.28 \mathrm{E}-07$ & & & 2.28E-07 & 2.94E-07 & & & 2.94E-07 \\
\hline $\mathrm{Ag}-108$ & 1.34E-06 & $2.12 \mathrm{E}-07$ & & $1.56 \mathrm{E}-06$ & 2.58E-06 & 5.34E-07 & & 3.11E-06 \\
\hline $\mathrm{Ag}-108 \mathrm{~m}$ & 1.56E-05 & $2.83 \mathrm{E}-06$ & & $1.85 \mathrm{E}-05$ & $2.92 \mathrm{E}-05$ & 6.97E-06 & & 3.61E-05 \\
\hline $\mathrm{Ag}-109 \mathrm{~m}$ & $2.89 \mathrm{E}-01$ & & & $2.89 \mathrm{E}-01$ & 3.63E-01 & & & 3.63E-01 \\
\hline $\mathrm{Ag}-110$ & 8.96E-04 & $1.26 \mathrm{E}-04$ & & $1.02 \mathrm{E}-03$ & 1.18E-03 & 3.18E-04 & & $1.50 \mathrm{E}-03$ \\
\hline $\mathrm{Ag}-110 \mathrm{~m}$ & $6.59 \mathrm{E}-02$ & $9.10 \mathrm{E}-03$ & $2.13 \mathrm{E}-02$ & $9.63 \mathrm{E}-02$ & 8.70E-02 & $2.29 \mathrm{E}-02$ & 5.33E-02 & 1.63E-01 \\
\hline$A m-241$ & $2.42 \mathrm{E}-01$ & $2.32 \mathrm{E}-02$ & 1.39E-02 & 2.79E-01 & 3.04E-01 & 6.06E-02 & 3.47E-02 & 3.99E-01 \\
\hline Am-242 & 1.80E-03 & & 3.08E-06 & $1.80 \mathrm{E}-03$ & 2.68E-03 & & 7.71E-06 & 2.69E-03 \\
\hline$A m-242 m$ & 1.77E-03 & 4.67E-05 & $3.10 \mathrm{E}-06$ & $1.82 \mathrm{E}-03$ & $2.46 \mathrm{E}-03$ & $2.62 \mathrm{E}-04$ & 7.75E-06 & 2.73E-03 \\
\hline$A m-243$ & 4.61E-04 & $6.40 \mathrm{E}-05$ & 1.07E-05 & 5.35E-04 & 7.56E-04 & 2.34E-04 & 2.67E-05 & 1.02E-03 \\
\hline
\end{tabular}


TEM-10200-1

$03 / 01 / 2012$

ENGINEERING CALCULATIONS AND ANALYSIS

Page 23 of 30

Rev. 06

Baseline Radionuclide Inventory for the Remote-Handled Low-Level Waste Disposal

Title: $\quad$ Facility for Use in the Facility Performance Assessment

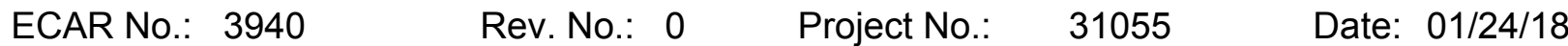

Table 16. (continued).

\begin{tabular}{|c|c|c|c|c|c|c|c|c|}
\hline \multirow[b]{2}{*}{ Nuclide } & \multicolumn{3}{|c|}{ 20-Year Inventory } & \multirow[b]{2}{*}{$\begin{array}{c}20-Y e a r \\
\text { Total }\end{array}$} & \multicolumn{4}{|c|}{ 50-Year Inventory } \\
\hline & AM & sc & Resin & & AM & sc & Resin & $\begin{array}{c}\text { 50-Year } \\
\text { Total }\end{array}$ \\
\hline Ar-37 & $6.27 \mathrm{E}-03$ & & & 6.27E-03 & $1.21 \mathrm{E}-02$ & & & 1.21E-02 \\
\hline Ar-39 & $3.24 \mathrm{E}-02$ & & & $3.24 \mathrm{E}-02$ & 4.95E-02 & & & 4.95E-02 \\
\hline Ar-42 & $2.75 \mathrm{E}-12$ & & & $2.75 \mathrm{E}-12$ & $5.51 \mathrm{E}-12$ & & & $5.51 \mathrm{E}-12$ \\
\hline As-76 & & & 8.28E-03 & $8.28 \mathrm{E}-03$ & & & 2.07E-02 & 2.07E-02 \\
\hline At-217 & $5.35 \mathrm{E}-08$ & & & $5.35 \mathrm{E}-08$ & $6.35 \mathrm{E}-08$ & & & $6.35 \mathrm{E}-08$ \\
\hline Ba-133 & 1.23E-03 & & & 1.23E-03 & $1.81 \mathrm{E}-03$ & & & 1.81E-03 \\
\hline $\mathrm{Ba}-136 \mathrm{~m}$ & $1.08 \mathrm{E}-10$ & & & $1.08 \mathrm{E}-10$ & $3.36 \mathrm{E}-10$ & & & $3.36 \mathrm{E}-10$ \\
\hline Ba-137m & $1.25 E+01$ & 4.13E-01 & $1.12 \mathrm{E}+01$ & $2.40 \mathrm{E}+01$ & $1.78 \mathrm{E}+01$ & $1.09 \mathrm{E}+00$ & $2.79 \mathrm{E}+01$ & 4.67E+01 \\
\hline $\mathrm{Ba}-140$ & 9.77E-09 & & $5.24 \mathrm{E}-02$ & 5.24E-02 & $2.83 \mathrm{E}-08$ & & 1.31E-01 & 1.31E-01 \\
\hline $\mathrm{Be}-10$ & $1.49 \mathrm{E}-04$ & 8.95E-06 & & $1.58 \mathrm{E}-04$ & $1.80 \mathrm{E}-04$ & $2.18 \mathrm{E}-05$ & & 2.01E-04 \\
\hline $\mathrm{Bi}-208$ & $2.64 \mathrm{E}-07$ & & & 2.64E-07 & $3.15 \mathrm{E}-07$ & & & 3.15E-07 \\
\hline $\mathrm{Bi}-210 \mathrm{~m}$ & 7.65E-07 & & & 7.65E-07 & $1.08 \mathrm{E}-06$ & & & 1.08E-06 \\
\hline $\mathrm{Bi}-211$ & $1.51 \mathrm{E}-06$ & 4.17E-06 & & $5.69 \mathrm{E}-06$ & $1.84 \mathrm{E}-06$ & 1.05E-05 & & $1.24 \mathrm{E}-05$ \\
\hline $\mathrm{Bi}-212$ & $2.00 \mathrm{E}-04$ & $1.98 \mathrm{E}-04$ & & $3.99 \mathrm{E}-04$ & $2.83 \mathrm{E}-04$ & $5.00 \mathrm{E}-04$ & & 7.83E-04 \\
\hline $\mathrm{Bi}-213$ & $5.35 \mathrm{E}-08$ & & & $5.35 \mathrm{E}-08$ & $6.35 \mathrm{E}-08$ & & & $6.35 \mathrm{E}-08$ \\
\hline $\mathrm{Bi}-214$ & $2.19 \mathrm{E}-11$ & & & $2.19 \mathrm{E}-11$ & $7.81 \mathrm{E}-11$ & & & $7.81 E-11$ \\
\hline Bk-249 & $6.50 \mathrm{E}-10$ & & & $6.50 \mathrm{E}-10$ & $2.00 \mathrm{E}-09$ & & & $2.00 \mathrm{E}-09$ \\
\hline$C-14$ & $2.05 E+02$ & $8.07 \mathrm{E}+00$ & $1.06 \mathrm{E}+00$ & $2.14 \mathrm{E}+02$ & $3.74 \mathrm{E}+02$ & $3.12 \mathrm{E}+01$ & $2.64 \mathrm{E}+00$ & $4.08 \mathrm{E}+02$ \\
\hline $\mathrm{Ca}-41$ & 7.32E-03 & & & $7.32 \mathrm{E}-03$ & 1.33E-02 & & & 1.33E-02 \\
\hline $\mathrm{Ca}-45$ & $5.24 \mathrm{E}-01$ & & $9.25 \mathrm{E}-04$ & $5.25 \mathrm{E}-01$ & 7.43E-01 & & $2.31 \mathrm{E}-03$ & 7.45E-01 \\
\hline Cd-109 & $2.89 \mathrm{E}-01$ & & $1.28 \mathrm{E}-02$ & $3.02 \mathrm{E}-01$ & 3.63E-01 & & $3.20 \mathrm{E}-02$ & $3.95 \mathrm{E}-01$ \\
\hline Cd-113m & $4.29 \mathrm{E}-02$ & $9.37 \mathrm{E}-01$ & & $9.80 \mathrm{E}-01$ & 5.59E-02 & $2.33 E+00$ & & $2.38 \mathrm{E}+00$ \\
\hline Cd-115m & 4.62E-05 & & & 4.62E-05 & 1.12E-04 & & & 1.12E-04 \\
\hline Ce-139 & 3.31E-04 & & & 3.31E-04 & $5.08 \mathrm{E}-04$ & & & $5.08 \mathrm{E}-04$ \\
\hline Ce-141 & $2.02 \mathrm{E}-03$ & $2.16 \mathrm{E}-05$ & $5.79 \mathrm{E}-01$ & 5.81E-01 & $4.92 \mathrm{E}-03$ & 5.44E-05 & $1.45 \mathrm{E}+00$ & $1.45 \mathrm{E}+00$ \\
\hline Ce-142 & 5.85E-07 & & & 5.85E-07 & $5.86 \mathrm{E}-07$ & & & 5.86E-07 \\
\hline $\mathrm{Ce}-144$ & $2.78 \mathrm{E}+01$ & 7.01E-01 & $2.88 \mathrm{E}+01$ & $5.73 E+01$ & $3.56 \mathrm{E}+01$ & $1.03 E+00$ & $7.20 \mathrm{E}+01$ & $1.09 \mathrm{E}+02$ \\
\hline Cf-249 & $5.78 \mathrm{E}-13$ & $3.13 \mathrm{E}-12$ & & 3.71E-12 & $1.38 \mathrm{E}-12$ & $8.63 \mathrm{E}-12$ & & $1.00 \mathrm{E}-11$ \\
\hline Cf-250 & $4.52 \mathrm{E}-11$ & & & $4.52 \mathrm{E}-11$ & $1.44 \mathrm{E}-10$ & & & $1.44 \mathrm{E}-10$ \\
\hline Cf-251 & $9.96 \mathrm{E}-16$ & $1.08 \mathrm{E}-13$ & & $1.09 \mathrm{E}-13$ & $3.26 \mathrm{E}-15$ & $3.15 \mathrm{E}-13$ & & $3.18 \mathrm{E}-13$ \\
\hline Cf-252 & $9.90 \mathrm{E}-11$ & & & $9.90 \mathrm{E}-11$ & $3.23 E-10$ & & & $3.23 E-10$ \\
\hline Cl-36 & 1.15E-01 & & & 1.15E-01 & $1.52 \mathrm{E}-01$ & & & $1.52 \mathrm{E}-01$ \\
\hline $\mathrm{Cm}-242$ & $9.96 \mathrm{E}+00$ & $2.50 \mathrm{E}-01$ & 1.71E-03 & $1.02 \mathrm{E}+01$ & $1.15 \mathrm{E}+01$ & $9.02 \mathrm{E}-01$ & $4.27 \mathrm{E}-03$ & $1.24 \mathrm{E}+01$ \\
\hline $\mathrm{Cm}-243$ & 1.15E-03 & $9.21 \mathrm{E}-05$ & $8.81 \mathrm{E}-06$ & $1.25 \mathrm{E}-03$ & $1.55 \mathrm{E}-03$ & 3.06E-04 & $2.20 \mathrm{E}-05$ & $1.88 \mathrm{E}-03$ \\
\hline $\mathrm{Cm}-244$ & $1.72 \mathrm{E}-02$ & $4.88 \mathrm{E}-03$ & $9.99 \mathrm{E}-03$ & $3.20 \mathrm{E}-02$ & $3.47 \mathrm{E}-02$ & 1.64E-02 & $2.50 \mathrm{E}-02$ & $7.60 \mathrm{E}-02$ \\
\hline $\mathrm{Cm}-245$ & $2.38 \mathrm{E}-07$ & 2.89E-07 & & $5.28 \mathrm{E}-07$ & 7.04E-07 & 7.62E-07 & & 1.47E-06 \\
\hline $\mathrm{Cm}-246$ & $1.72 \mathrm{E}-07$ & 1.80E-07 & & 3.52E-07 & 5.37E-07 & 5.04E-07 & & $1.04 \mathrm{E}-06$ \\
\hline $\mathrm{Cm}-247$ & $1.50 \mathrm{E}-15$ & $1.62 \mathrm{E}-13$ & & $1.64 \mathrm{E}-13$ & $4.91 \mathrm{E}-15$ & $4.72 \mathrm{E}-13$ & & 4.77E-13 \\
\hline $\mathrm{Cm}-248$ & $4.75 \mathrm{E}-15$ & $5.14 \mathrm{E}-13$ & & $5.18 \mathrm{E}-13$ & $1.55 \mathrm{E}-14$ & 1.50E-12 & & $1.51 \mathrm{E}-12$ \\
\hline Co-57 & $4.04 \mathrm{E}-04$ & 7.16E-04 & $1.56 \mathrm{E}-01$ & 1.57E-01 & $1.02 \mathrm{E}-03$ & 1.80E-03 & $3.89 \mathrm{E}-01$ & 3.92E-01 \\
\hline Co-58 & $2.51 \mathrm{E}+04$ & $1.21 \mathrm{E}+03$ & $5.21 \mathrm{E}+01$ & $2.64 \mathrm{E}+04$ & $4.24 \mathrm{E}+04$ & $2.83 E+03$ & $1.30 \mathrm{E}+02$ & $4.54 \mathrm{E}+04$ \\
\hline
\end{tabular}


TEM-10200-1

$03 / 01 / 2012$

ENGINEERING CALCULATIONS AND ANALYSIS

Page 24 of 30

Rev. 06

Baseline Radionuclide Inventory for the Remote-Handled Low-Level Waste Disposal

Title: $\quad$ Facility for Use in the Facility Performance Assessment

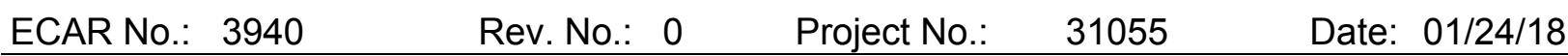

Table 16. (continued).

\begin{tabular}{|c|c|c|c|c|c|c|c|c|}
\hline \multirow[b]{2}{*}{ Nuclide } & \multicolumn{3}{|c|}{ 20-Year Inventory } & \multirow[b]{2}{*}{$\begin{array}{c}20-\text { Year } \\
\text { Total }\end{array}$} & \multicolumn{4}{|c|}{ 50-Year Inventory } \\
\hline & AM & sc & Resin & & AM & SC & Resin & $\begin{array}{c}50-Y e a r \\
\text { Total }\end{array}$ \\
\hline Co-60 & $3.07 E+05$ & $7.34 \mathrm{E}+02$ & $1.85 E+03$ & $3.09 \mathrm{E}+05$ & $6.49 \mathrm{E}+05$ & $2.71 \mathrm{E}+03$ & $4.63 \mathrm{E}+03$ & $6.56 E+05$ \\
\hline $\mathrm{Cr}-51$ & $4.92 E+03$ & $1.58 \mathrm{E}+01$ & $6.40 \mathrm{E}+02$ & $5.57 \mathrm{E}+03$ & $1.24 \mathrm{E}+04$ & $1.93 E+01$ & $1.60 \mathrm{E}+03$ & $1.40 \mathrm{E}+04$ \\
\hline Cs-134 & $1.03 E+02$ & $1.27 \mathrm{E}-01$ & $2.40 \mathrm{E}+00$ & $1.05 \mathrm{E}+02$ & $1.61 \mathrm{E}+02$ & $3.90 \mathrm{E}-01$ & $6.00 \mathrm{E}+00$ & $1.67 \mathrm{E}+02$ \\
\hline Cs-135 & $1.55 \mathrm{E}-04$ & $2.60 \mathrm{E}-02$ & & 2.61E-02 & $2.40 \mathrm{E}-04$ & 2.60E-02 & & 2.62E-02 \\
\hline Cs-136 & $9.69 \mathrm{E}-10$ & & & $9.69 \mathrm{E}-10$ & 3.03E-09 & & & 3.03E-09 \\
\hline Cs-137 & $1.32 E+01$ & $9.18 \mathrm{E}+02$ & $1.43 \mathrm{E}+01$ & $9.45 \mathrm{E}+02$ & $1.88 \mathrm{E}+01$ & $9.20 \mathrm{E}+02$ & $3.57 \mathrm{E}+01$ & $9.74 \mathrm{E}+02$ \\
\hline Eu-152 & $1.28 \mathrm{E}-01$ & & $4.01 \mathrm{E}+00$ & $4.14 \mathrm{E}+00$ & 1.87E-01 & & $1.00 \mathrm{E}+01$ & $1.02 \mathrm{E}+01$ \\
\hline Eu-154 & 4.59E-01 & $3.30 \mathrm{E}+00$ & $1.18 \mathrm{E}+01$ & $1.56 \mathrm{E}+01$ & $6.81 \mathrm{E}-01$ & $3.31 \mathrm{E}+00$ & $2.95 \mathrm{E}+01$ & $3.35 \mathrm{E}+01$ \\
\hline Eu-155 & $1.70 \mathrm{E}-01$ & $1.56 \mathrm{E}-01$ & $1.33 \mathrm{E}+00$ & $1.65 \mathrm{E}+00$ & $2.45 \mathrm{E}-01$ & 1.63E-01 & $3.32 \mathrm{E}+00$ & $3.73 \mathrm{E}+00$ \\
\hline Eu-156 & $2.59 \mathrm{E}-08$ & & & $2.59 \mathrm{E}-08$ & $8.11 \mathrm{E}-08$ & & & 8.11E-08 \\
\hline $\mathrm{Fe}-55$ & $1.91 \mathrm{E}+05$ & $1.30 \mathrm{E}+03$ & $4.03 E+03$ & $1.97 \mathrm{E}+05$ & $3.67 \mathrm{E}+05$ & $4.92 \mathrm{E}+03$ & $1.01 \mathrm{E}+04$ & $3.82 E+05$ \\
\hline Fe-59 & $5.96 \mathrm{E}+02$ & $6.41 \mathrm{E}-01$ & $2.06 \mathrm{E}+01$ & $6.17 \mathrm{E}+02$ & $1.50 \mathrm{E}+03$ & $1.76 \mathrm{E}+00$ & $5.14 \mathrm{E}+01$ & $1.55 \mathrm{E}+03$ \\
\hline Fr-221 & $5.35 \mathrm{E}-08$ & & & $5.35 \mathrm{E}-08$ & $6.35 \mathrm{E}-08$ & & & $6.35 \mathrm{E}-08$ \\
\hline Fr-223 & 1.56E-08 & & & $1.56 \mathrm{E}-08$ & 1.56E-08 & & & $1.56 \mathrm{E}-08$ \\
\hline Gd-153 & $7.80 \mathrm{E}-03$ & & 1.31E-01 & 1.39E-01 & 1.02E-02 & & $3.28 \mathrm{E}-01$ & 3.39E-01 \\
\hline $\mathrm{H}-3$ & $1.98 E+03$ & $3.49 \mathrm{E}-05$ & $3.86 \mathrm{E}+00$ & $1.99 \mathrm{E}+03$ & $3.93 E+03$ & 3.49E-05 & $9.65 \mathrm{E}+00$ & $3.94 \mathrm{E}+03$ \\
\hline Hf-175 & $1.52 E+02$ & & $1.48 \mathrm{E}+00$ & $1.53 E+02$ & $2.00 \mathrm{E}+02$ & & $3.71 \mathrm{E}+00$ & $2.04 \mathrm{E}+02$ \\
\hline Hf-178m & 4.01E-08 & & & 4.01E-08 & 4.01E-08 & & & 4.01E-08 \\
\hline Hf-181 & $6.21 \mathrm{E}+01$ & $1.71 \mathrm{E}-01$ & $6.42 \mathrm{E}+00$ & $6.87 E+01$ & $1.25 \mathrm{E}+02$ & 4.91E-01 & $1.60 \mathrm{E}+01$ & $1.42 E+02$ \\
\hline Hf-182 & 5.80E-05 & & & $5.80 \mathrm{E}-05$ & 1.14E-04 & & & 1.14E-04 \\
\hline Ho-166m & $2.86 \mathrm{E}-08$ & & & $2.86 \mathrm{E}-08$ & $5.48 \mathrm{E}-08$ & & & $5.48 \mathrm{E}-08$ \\
\hline I-129 & $8.01 \mathrm{E}-06$ & $5.05 \mathrm{E}-04$ & $5.33 \mathrm{E}-02$ & $5.38 \mathrm{E}-02$ & $1.32 \mathrm{E}-05$ & $5.46 \mathrm{E}-04$ & 1.33E-01 & $1.34 \mathrm{E}-01$ \\
\hline I-131 & & & 1.37E-02 & 1.37E-02 & & & $3.42 \mathrm{E}-02$ & 3.42E-02 \\
\hline I-132 & & & $2.18 \mathrm{E}-03$ & 2.18E-03 & & & $5.46 \mathrm{E}-03$ & $5.46 \mathrm{E}-03$ \\
\hline I-133 & & & $8.66 \mathrm{E}-03$ & $8.66 \mathrm{E}-03$ & & & $2.16 \mathrm{E}-02$ & 2.16E-02 \\
\hline In-113m & $3.69 E+03$ & & 9.33E-01 & $3.69 \mathrm{E}+03$ & $4.99 \mathrm{E}+03$ & & $2.33 \mathrm{E}+00$ & $4.99 E+03$ \\
\hline In-114 & $2.28 \mathrm{E}+00$ & & 3.07E-05 & $2.28 \mathrm{E}+00$ & $4.15 \mathrm{E}+00$ & & 7.67E-05 & $4.15 E+00$ \\
\hline In-114m & $2.38 E+00$ & & $3.20 \mathrm{E}-05$ & $2.38 \mathrm{E}+00$ & $4.34 \mathrm{E}+00$ & & $8.01 \mathrm{E}-05$ & $4.34 \mathrm{E}+00$ \\
\hline In-115 & $2.02 \mathrm{E}-15$ & $7.52 \mathrm{E}-13$ & & $7.54 \mathrm{E}-13$ & $4.04 \mathrm{E}-15$ & $7.52 \mathrm{E}-13$ & & $7.56 \mathrm{E}-13$ \\
\hline In-115m & $3.97 \mathrm{E}-10$ & & & $3.97 \mathrm{E}-10$ & 1.12E-09 & & & 1.12E-09 \\
\hline Ir-192 & $5.45 \mathrm{E}-02$ & 1.25E-09 & & $5.45 \mathrm{E}-02$ & $9.33 \mathrm{E}-02$ & 3.14E-09 & & 9.33E-02 \\
\hline Ir-192m & 1.07E-05 & & & 1.07E-05 & 1.11E-05 & & & 1.11E-05 \\
\hline Ir-194 & 3.46E-09 & & & $3.46 \mathrm{E}-09$ & 4.05E-09 & & & $4.05 \mathrm{E}-09$ \\
\hline Ir-194m & $6.14 \mathrm{E}-03$ & & & $6.14 \mathrm{E}-03$ & $9.82 \mathrm{E}-03$ & & & $9.82 \mathrm{E}-03$ \\
\hline$K-40$ & $6.33 \mathrm{E}-07$ & & 5.06E-04 & 5.06E-04 & $1.09 \mathrm{E}-06$ & & $1.26 \mathrm{E}-03$ & 1.27E-03 \\
\hline$K-42$ & $2.75 \mathrm{E}-12$ & & & $2.75 \mathrm{E}-12$ & $5.51 \mathrm{E}-12$ & & & $5.51 \mathrm{E}-12$ \\
\hline $\mathrm{Kr}-81$ & $1.70 \mathrm{E}-12$ & & & $1.70 \mathrm{E}-12$ & $4.44 \mathrm{E}-12$ & & & $4.44 \mathrm{E}-12$ \\
\hline $\mathrm{Kr}-85$ & $6.51 \mathrm{E}-01$ & $4.90 \mathrm{E}+01$ & 7.65E-01 & $5.04 \mathrm{E}+01$ & $9.29 \mathrm{E}-01$ & $1.00 \mathrm{E}+02$ & $1.91 \mathrm{E}+00$ & $1.03 E+02$ \\
\hline La-137 & $2.38 \mathrm{E}-06$ & & & $2.38 \mathrm{E}-06$ & $2.38 \mathrm{E}-06$ & & & $2.38 \mathrm{E}-06$ \\
\hline La-140 & $1.20 \mathrm{E}-08$ & & $1.18 \mathrm{E}-01$ & 1.18E-01 & 3.33E-08 & & 2.96E-01 & $2.96 \mathrm{E}-01$ \\
\hline Lu-176 & 7.76E-09 & & & 7.76E-09 & 1.49E-08 & & & 1.49E-08 \\
\hline
\end{tabular}


TEM-10200-1

$03 / 01 / 2012$

ENGINEERING CALCULATIONS AND ANALYSIS

Page 25 of 30

Rev. 06

Baseline Radionuclide Inventory for the Remote-Handled Low-Level Waste Disposal

Title: $\quad$ Facility for Use in the Facility Performance Assessment

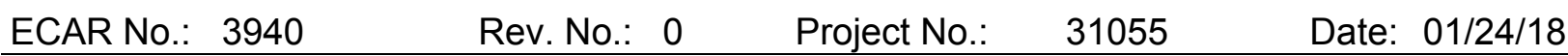

Table 16. (continued).

\begin{tabular}{|c|c|c|c|c|c|c|c|c|}
\hline \multirow[b]{2}{*}{ Nuclide } & \multicolumn{3}{|c|}{ 20-Year Inventory } & \multirow[b]{2}{*}{$\begin{array}{c}20-\text { Year } \\
\text { Total }\end{array}$} & \multicolumn{4}{|c|}{ 50-Year Inventory } \\
\hline & AM & sc & Resin & & AM & sc & Resin & $\begin{array}{c}50-Y e a r \\
\text { Total }\end{array}$ \\
\hline Lu-177 & 1.06E-02 & & & $1.06 \mathrm{E}-02$ & $1.53 \mathrm{E}-02$ & & & 1.53E-02 \\
\hline Lu-177m & 4.82E-02 & & & 4.82E-02 & 6.97E-02 & & & $6.97 \mathrm{E}-02$ \\
\hline$M n-54$ & $2.02 \mathrm{E}+04$ & $1.52 \mathrm{E}+02$ & $2.88 \mathrm{E}+01$ & $2.04 \mathrm{E}+04$ & $4.87 \mathrm{E}+04$ & $3.61 \mathrm{E}+02$ & $7.19 \mathrm{E}+01$ & $4.91 E+04$ \\
\hline Mo-93 & $2.55 E+01$ & 3.19E-01 & & $2.58 \mathrm{E}+01$ & $2.72 E+01$ & $3.19 \mathrm{E}-01$ & & $2.75 \mathrm{E}+01$ \\
\hline Mo-99 & & & $3.28 \mathrm{E}-01$ & $3.28 \mathrm{E}-01$ & & & $8.20 \mathrm{E}-01$ & 8.20E-01 \\
\hline $\mathrm{Na}-24$ & & & $3.68 \mathrm{E}-06$ & $3.68 \mathrm{E}-06$ & & & $9.21 \mathrm{E}-06$ & $9.21 \mathrm{E}-06$ \\
\hline $\mathrm{Nb}-92$ & 4.43E-06 & & & 4.43E-06 & $5.88 \mathrm{E}-06$ & & & $5.88 \mathrm{E}-06$ \\
\hline $\mathrm{Nb}-93 \mathrm{~m}$ & $4.94 \mathrm{E}+02$ & $7.62 \mathrm{E}+01$ & 8.47E-05 & $5.70 \mathrm{E}+02$ & $5.91 \mathrm{E}+02$ & $1.03 \mathrm{E}+02$ & $2.12 \mathrm{E}-04$ & $6.94 \mathrm{E}+02$ \\
\hline $\mathrm{Nb}-94$ & $5.60 \mathrm{E}+01$ & $2.28 \mathrm{E}-01$ & $8.48 \mathrm{E}-01$ & $5.71 \mathrm{E}+01$ & $1.05 \mathrm{E}+02$ & $6.90 \mathrm{E}-01$ & $2.12 \mathrm{E}+00$ & $1.08 \mathrm{E}+02$ \\
\hline $\mathrm{Nb}-95$ & $7.90 E+04$ & $5.33 E+01$ & $2.55 E+01$ & $7.91 \mathrm{E}+04$ & $1.11 \mathrm{E}+05$ & $1.35 \mathrm{E}+02$ & $6.38 \mathrm{E}+01$ & 1.11E+05 \\
\hline $\mathrm{Nb}-95 \mathrm{~m}$ & $4.23 E+02$ & $3.65 \mathrm{E}-07$ & $1.14 \mathrm{E}-04$ & $4.23 E+02$ & $5.91 \mathrm{E}+02$ & $9.20 \mathrm{E}-07$ & $2.84 \mathrm{E}-04$ & $5.91 E+02$ \\
\hline $\mathrm{Nd}-147$ & $1.38 \mathrm{E}-10$ & & & $1.38 \mathrm{E}-10$ & $4.20 \mathrm{E}-10$ & & & $4.20 \mathrm{E}-10$ \\
\hline $\mathrm{Ni}-59$ & $1.80 E+03$ & $4.84 \mathrm{E}+00$ & $1.19 E+01$ & $1.82 \mathrm{E}+03$ & $3.05 \mathrm{E}+03$ & $1.54 \mathrm{E}+01$ & $2.98 \mathrm{E}+01$ & $3.10 E+03$ \\
\hline $\mathrm{Ni}-63$ & 2.17E+05 & $4.21 \mathrm{E}+02$ & $1.44 \mathrm{E}+03$ & $2.18 \mathrm{E}+05$ & $3.73 E+05$ & $1.51 \mathrm{E}+03$ & $3.60 \mathrm{E}+03$ & $3.78 \mathrm{E}+05$ \\
\hline $\mathrm{Np}-235$ & $1.59 \mathrm{E}-09$ & & & 1.59E-09 & $2.46 \mathrm{E}-09$ & & & 2.46E-09 \\
\hline Np-236 & $1.09 \mathrm{E}-11$ & & & $1.09 \mathrm{E}-11$ & $1.23 \mathrm{E}-11$ & & & 1.23E-11 \\
\hline Np-237 & $6.25 \mathrm{E}-06$ & $5.82 \mathrm{E}-04$ & $1.07 \mathrm{E}-04$ & $6.95 \mathrm{E}-04$ & 8.39E-06 & 5.82E-04 & $2.66 \mathrm{E}-04$ & 8.57E-04 \\
\hline Np-238 & $8.10 \mathrm{E}-06$ & & $1.39 \mathrm{E}-08$ & 8.11E-06 & $1.20 \mathrm{E}-05$ & & $3.49 \mathrm{E}-08$ & 1.21E-05 \\
\hline Np-239 & $5.08 \mathrm{E}-04$ & & 1.07E-05 & 5.19E-04 & $9.42 \mathrm{E}-04$ & & $2.67 \mathrm{E}-05$ & $9.69 \mathrm{E}-04$ \\
\hline Np-240m & $2.65 \mathrm{E}-13$ & & & $2.65 \mathrm{E}-13$ & 8.77E-13 & & & 8.77E-13 \\
\hline Os-185 & 7.74E-05 & & & 7.74E-05 & $1.84 \mathrm{E}-04$ & & & $1.84 \mathrm{E}-04$ \\
\hline Os-191 & 8.09E-09 & & & 8.09E-09 & $2.69 \mathrm{E}-08$ & & & $2.69 \mathrm{E}-08$ \\
\hline Os-194 & $3.44 \mathrm{E}-09$ & & & $3.44 \mathrm{E}-09$ & 4.03E-09 & & & 4.03E-09 \\
\hline P-32 & $3.90 \mathrm{E}-06$ & & $1.42 \mathrm{E}-08$ & $3.92 \mathrm{E}-06$ & $6.55 \mathrm{E}-06$ & & $3.55 E-08$ & 6.58E-06 \\
\hline P-33 & $3.95 \mathrm{E}-02$ & & & $3.95 \mathrm{E}-02$ & $5.82 \mathrm{E}-02$ & & & 5.82E-02 \\
\hline Pa-231 & $6.68 \mathrm{E}-06$ & & & $6.68 \mathrm{E}-06$ & $8.74 \mathrm{E}-06$ & & & 8.74E-06 \\
\hline $\mathrm{Pa}-233$ & 1.23E-04 & 2.07E-17 & & $1.23 E-04$ & $1.92 \mathrm{E}-04$ & $5.21 \mathrm{E}-17$ & & $1.92 \mathrm{E}-04$ \\
\hline Pa-234 & $1.39 \mathrm{E}-07$ & & & 1.39E-07 & $3.26 \mathrm{E}-07$ & & & 3.26E-07 \\
\hline Pa-234m & $1.35 \mathrm{E}-04$ & & & $1.35 \mathrm{E}-04$ & $2.84 \mathrm{E}-04$ & & & $2.84 \mathrm{E}-04$ \\
\hline $\mathrm{Pb}-204$ & $2.36 \mathrm{E}-13$ & & & $2.36 \mathrm{E}-13$ & $4.72 \mathrm{E}-13$ & & & $4.72 \mathrm{E}-13$ \\
\hline $\mathrm{Pb}-205$ & $4.90 \mathrm{E}-07$ & & & 4.90E-07 & $8.25 \mathrm{E}-07$ & & & 8.25E-07 \\
\hline $\mathrm{Pb}-209$ & 5.35E-08 & & & $5.35 \mathrm{E}-08$ & $6.35 \mathrm{E}-08$ & & & 6.35E-08 \\
\hline $\mathrm{Pb}-210$ & & $2.89 \mathrm{E}-12$ & & $2.89 \mathrm{E}-12$ & & $2.89 \mathrm{E}-12$ & & $2.89 \mathrm{E}-12$ \\
\hline $\mathrm{Pb}-211$ & $1.51 \mathrm{E}-06$ & $4.15 \mathrm{E}-06$ & & 5.66E-06 & 1.84E-06 & 1.05E-05 & & 1.23E-05 \\
\hline $\mathrm{Pb}-212$ & $2.02 \mathrm{E}-04$ & $1.98 \mathrm{E}-04$ & & 4.00E-04 & $2.85 \mathrm{E}-04$ & $5.00 \mathrm{E}-04$ & & 7.84E-04 \\
\hline $\mathrm{Pb}-214$ & $2.19 \mathrm{E}-11$ & & & $2.19 \mathrm{E}-11$ & $7.81 \mathrm{E}-11$ & & & $7.81 \mathrm{E}-11$ \\
\hline Pd-107 & $2.76 \mathrm{E}-05$ & $4.35 \mathrm{E}-04$ & & 4.63E-04 & $3.97 \mathrm{E}-05$ & 4.35E-04 & & 4.75E-04 \\
\hline Pm-145 & $5.31 \mathrm{E}-11$ & & & 5.31E-11 & $1.58 \mathrm{E}-10$ & & & $1.58 \mathrm{E}-10$ \\
\hline Pm-146 & 6.01E-08 & & & $6.01 \mathrm{E}-08$ & 8.36E-08 & & & 8.36E-08 \\
\hline Pm-147 & $1.44 \mathrm{E}+01$ & $2.78 \mathrm{E}-01$ & $3.76 \mathrm{E}+01$ & $5.23 E+01$ & $1.94 \mathrm{E}+01$ & 4.27E-01 & $9.40 \mathrm{E}+01$ & $1.14 \mathrm{E}+02$ \\
\hline Pm-148 & $9.18 \mathrm{E}-06$ & & & 9.18E-06 & 2.35E-05 & & & 2.35E-05 \\
\hline
\end{tabular}


TEM-10200-1

$03 / 01 / 2012$

ENGINEERING CALCULATIONS AND ANALYSIS

Page 26 of 30

Rev. 06

Baseline Radionuclide Inventory for the Remote-Handled Low-Level Waste Disposal

Title: $\quad$ Facility for Use in the Facility Performance Assessment

$\begin{array}{lllll}\text { ECAR No.: } 3940 \quad \text { Rev. No.: } & 0 & \text { Project No.: } & 31055 & \text { Date: } 01 / 24 / 18\end{array}$

Table 16. (continued).

\begin{tabular}{|c|c|c|c|c|c|c|c|c|}
\hline \multirow[b]{2}{*}{ Nuclide } & \multicolumn{3}{|c|}{ 20-Year Inventory } & \multirow[b]{2}{*}{$\begin{array}{c}20-Y e a r \\
\text { Total }\end{array}$} & \multicolumn{4}{|c|}{ 50-Year Inventory } \\
\hline & AM & sc & Resin & & AM & sc & Resin & $\begin{array}{c}50-\text { Year } \\
\text { Total }\end{array}$ \\
\hline Pm-148m & 1.67E-04 & & & 1.67E-04 & 4.34E-04 & & & 4.34E-04 \\
\hline Po-210 & $2.84 \mathrm{E}-01$ & & & $2.84 \mathrm{E}-01$ & 3.63E-01 & & & 3.63E-01 \\
\hline Po-211 & 3.02E-09 & & & $3.02 \mathrm{E}-09$ & $3.02 \mathrm{E}-09$ & & & 3.02E-09 \\
\hline Po-212 & $1.29 \mathrm{E}-04$ & & & 1.29E-04 & $1.82 E-04$ & & & 1.82E-04 \\
\hline Po-213 & $5.23 \mathrm{E}-08$ & & & 5.23E-08 & $6.20 \mathrm{E}-08$ & & & $6.20 \mathrm{E}-08$ \\
\hline Po-214 & 2.19E-11 & & & $2.19 \mathrm{E}-11$ & $7.81 \mathrm{E}-11$ & & & $7.81 \mathrm{E}-11$ \\
\hline Po-215 & $1.51 \mathrm{E}-06$ & & & $1.51 \mathrm{E}-06$ & $1.84 \mathrm{E}-06$ & & & $1.84 \mathrm{E}-06$ \\
\hline Po-216 & $2.02 \mathrm{E}-04$ & & & 2.02E-04 & $2.85 \mathrm{E}-04$ & & & 2.85E-04 \\
\hline Po-218 & $2.19 \mathrm{E}-11$ & & & $2.19 \mathrm{E}-11$ & $7.81 \mathrm{E}-11$ & & & $7.81 \mathrm{E}-11$ \\
\hline Pr-143 & $3.21 \mathrm{E}-08$ & & & 3.21E-08 & 8.64E-08 & & & 8.64E-08 \\
\hline Pr-144 & $2.84 \mathrm{E}+01$ & 7.35E-03 & $1.52 \mathrm{E}+01$ & $4.36 \mathrm{E}+01$ & $3.63 E+01$ & $1.85 \mathrm{E}-02$ & $3.80 \mathrm{E}+01$ & $7.43 E+01$ \\
\hline Pr-144m & 3.97E-01 & & & 3.97E-01 & $5.08 \mathrm{E}-01$ & & & $5.08 \mathrm{E}-01$ \\
\hline Pt-193 & $6.64 \mathrm{E}-04$ & 2.98E-09 & & 6.64E-04 & $9.00 \mathrm{E}-04$ & 7.27E-09 & & $9.00 \mathrm{E}-04$ \\
\hline $\mathrm{Pu}-236$ & $5.15 \mathrm{E}-07$ & 1.77E-07 & & 6.92E-07 & $6.99 \mathrm{E}-07$ & 1.77E-07 & & 8.76E-07 \\
\hline Pu-237 & $3.46 \mathrm{E}-08$ & & & $3.46 \mathrm{E}-08$ & 4.66E-08 & & & 4.66E-08 \\
\hline $\mathrm{Pu}-238$ & $1.76 \mathrm{E}-01$ & $3.55 \mathrm{E}-02$ & $1.56 \mathrm{E}-01$ & 3.68E-01 & 2.46E-01 & $6.59 \mathrm{E}-02$ & $3.91 \mathrm{E}-01$ & 7.03E-01 \\
\hline Pu-239 & $2.13 E-01$ & 3.15E-01 & 2.89E-02 & $5.56 \mathrm{E}-01$ & $3.02 \mathrm{E}-01$ & 3.25E-01 & $7.23 E-02$ & $6.99 \mathrm{E}-01$ \\
\hline Pu-240 & 1.72E-01 & $2.28 \mathrm{E}-03$ & 2.01E-03 & 1.76E-01 & $2.38 \mathrm{E}-01$ & 3.16E-03 & $5.04 \mathrm{E}-03$ & 2.46E-01 \\
\hline Pu-241 & $1.95 \mathrm{E}+01$ & 2.07E-01 & 4.45E-02 & $1.97 \mathrm{E}+01$ & $2.58 E+01$ & $1.00 \mathrm{E}+00$ & 1.11E-01 & $2.69 \mathrm{E}+01$ \\
\hline $\mathrm{Pu}-242$ & $2.13 \mathrm{E}-04$ & 1.22E-05 & $1.62 \mathrm{E}-06$ & 2.27E-04 & 3.13E-04 & 5.04E-05 & 4.04E-06 & 3.67E-04 \\
\hline $\mathrm{Pu}-244$ & $1.22 \mathrm{E}-13$ & $3.89 \mathrm{E}-13$ & & 5.11E-13 & $2.40 \mathrm{E}-13$ & $1.04 \mathrm{E}-12$ & & $1.28 \mathrm{E}-12$ \\
\hline Ra-223 & $1.51 \mathrm{E}-06$ & & & $1.51 \mathrm{E}-06$ & $1.84 \mathrm{E}-06$ & & & $1.84 \mathrm{E}-06$ \\
\hline Ra-224 & $2.02 \mathrm{E}-04$ & & & 2.02E-04 & $2.85 \mathrm{E}-04$ & & & $2.85 \mathrm{E}-04$ \\
\hline Ra-225 & $5.35 \mathrm{E}-08$ & & & $5.35 \mathrm{E}-08$ & $6.35 \mathrm{E}-08$ & & & $6.35 \mathrm{E}-08$ \\
\hline Ra-226 & $2.19 \mathrm{E}-11$ & $9.53 \mathrm{E}-12$ & & $3.14 \mathrm{E}-11$ & $7.81 \mathrm{E}-11$ & $9.53 \mathrm{E}-12$ & & 8.76E-11 \\
\hline Ra-228 & $2.28 \mathrm{E}-07$ & & & 2.28E-07 & $2.94 \mathrm{E}-07$ & & & 2.94E-07 \\
\hline Rb-86 & $1.40 \mathrm{E}-06$ & & & $1.40 \mathrm{E}-06$ & $2.20 \mathrm{E}-06$ & & & $2.20 \mathrm{E}-06$ \\
\hline Rb-87 & $1.28 \mathrm{E}-06$ & & & $1.28 \mathrm{E}-06$ & $1.28 \mathrm{E}-06$ & & & $1.28 \mathrm{E}-06$ \\
\hline $\operatorname{Re}-186$ & & & $2.54 \mathrm{E}-06$ & $2.54 \mathrm{E}-06$ & & & $6.35 \mathrm{E}-06$ & $6.35 \mathrm{E}-06$ \\
\hline $\operatorname{Re}-187$ & 1.15E-05 & 2.71E-06 & 3.58E-01 & $3.58 \mathrm{E}-01$ & 2.11E-05 & $6.58 \mathrm{E}-06$ & 8.94E-01 & 8.94E-01 \\
\hline $\operatorname{Re}-188$ & 1.27E-01 & & $2.45 \mathrm{E}-01$ & 3.71E-01 & $1.89 \mathrm{E}-01$ & & $6.12 \mathrm{E}-01$ & 8.01E-01 \\
\hline Rh-102 & $1.02 \mathrm{E}-04$ & 1.75E-08 & & 1.02E-04 & $1.22 \mathrm{E}-04$ & 4.40E-08 & & 1.22E-04 \\
\hline Rh-103m & 8.68E-03 & & 9.79E-03 & $1.85 \mathrm{E}-02$ & $2.21 \mathrm{E}-02$ & & $2.45 \mathrm{E}-02$ & 4.66E-02 \\
\hline Rh-106 & $2.98 \mathrm{E}+01$ & $3.44 \mathrm{E}+01$ & 3.18E-01 & $6.45 E+01$ & $3.80 E+01$ & $8.66 \mathrm{E}+01$ & 7.95E-01 & $1.25 E+02$ \\
\hline Rn-219 & $1.51 \mathrm{E}-06$ & & & $1.51 \mathrm{E}-06$ & $1.84 \mathrm{E}-06$ & & & $1.84 \mathrm{E}-06$ \\
\hline$R n-220$ & $2.02 \mathrm{E}-04$ & & & $2.02 \mathrm{E}-04$ & $2.85 \mathrm{E}-04$ & & & $2.85 \mathrm{E}-04$ \\
\hline Rn-222 & $2.19 \mathrm{E}-11$ & & & $2.19 \mathrm{E}-11$ & $7.81 \mathrm{E}-11$ & & & $7.81 \mathrm{E}-11$ \\
\hline Ru-103 & 8.75E-03 & & 2.37E-01 & $2.45 \mathrm{E}-01$ & $2.23 \mathrm{E}-02$ & & 5.92E-01 & 6.14E-01 \\
\hline Ru-106 & $2.91 \mathrm{E}+01$ & $4.38 \mathrm{E}+01$ & $3.18 \mathrm{E}-01$ & $7.32 \mathrm{E}+01$ & $3.72 E+01$ & $1.09 \mathrm{E}+02$ & 7.95E-01 & $1.48 \mathrm{E}+02$ \\
\hline S-35 & $3.70 \mathrm{E}+00$ & & & $3.70 \mathrm{E}+00$ & $5.51 \mathrm{E}+00$ & & & $5.51 \mathrm{E}+00$ \\
\hline Sb-124 & $2.20 \mathrm{E}+00$ & 1.39E-04 & $1.94 \mathrm{E}-01$ & $2.39 E+00$ & $4.40 \mathrm{E}+00$ & $3.50 \mathrm{E}-04$ & 4.84E-01 & $4.89 E+00$ \\
\hline
\end{tabular}


TEM-10200-1

$03 / 01 / 2012$

ENGINEERING CALCULATIONS AND ANALYSIS

Page 27 of 30

Rev. 06

Baseline Radionuclide Inventory for the Remote-Handled Low-Level Waste Disposal

Title: $\quad$ Facility for Use in the Facility Performance Assessment

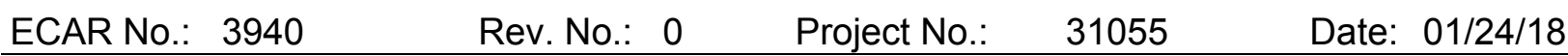

Table 16. (continued).

\begin{tabular}{|c|c|c|c|c|c|c|c|c|}
\hline \multirow[b]{2}{*}{ Nuclide } & \multicolumn{3}{|c|}{ 20-Year Inventory } & \multirow[b]{2}{*}{$\begin{array}{c}20-\text { Year } \\
\text { Total }\end{array}$} & \multicolumn{4}{|c|}{ 50-Year Inventory } \\
\hline & AM & SC & Resin & & AM & SC & Resin & $\begin{array}{c}50-Y e a r \\
\text { Total }\end{array}$ \\
\hline Sb-125 & $2.27 E+04$ & $8.15 E+00$ & $3.29 E+01$ & $2.28 \mathrm{E}+04$ & $3.45 \mathrm{E}+04$ & $2.55 \mathrm{E}+01$ & $8.22 \mathrm{E}+01$ & $3.46 E+04$ \\
\hline Sb-126 & $1.84 \mathrm{E}-05$ & $6.77 \mathrm{E}-03$ & & $6.79 \mathrm{E}-03$ & $2.63 E-05$ & $1.71 \mathrm{E}-02$ & & 1.71E-02 \\
\hline Sb-126m & 1.32E-04 & $5.03 E+00$ & & $5.03 E+00$ & $1.89 \mathrm{E}-04$ & $1.27 \mathrm{E}+01$ & & $1.27 \mathrm{E}+01$ \\
\hline Sc-46 & $3.90 E+00$ & $2.87 \mathrm{E}-08$ & 2.39E-01 & $4.14 \mathrm{E}+00$ & $5.10 \mathrm{E}+00$ & $7.24 \mathrm{E}-08$ & 5.99E-01 & $5.70 \mathrm{E}+00$ \\
\hline Se-75 & $1.27 E+01$ & & 3.23E-02 & $1.27 \mathrm{E}+01$ & $1.74 \mathrm{E}+01$ & & 8.08E-02 & $1.74 \mathrm{E}+01$ \\
\hline Se-79 & 1.73E-03 & 7.82E-03 & 3.64E-05 & $9.58 \mathrm{E}-03$ & $3.37 \mathrm{E}-03$ & 7.82E-03 & $9.09 \mathrm{E}-05$ & 1.13E-02 \\
\hline Si-32 & 3.21E-07 & & 7.83E-09 & $3.28 \mathrm{E}-07$ & $6.43 \mathrm{E}-07$ & & 1.96E-08 & 6.63E-07 \\
\hline Sm-147 & $1.38 \mathrm{E}-10$ & & & $1.38 \mathrm{E}-10$ & $4.28 \mathrm{E}-10$ & & & $4.28 \mathrm{E}-10$ \\
\hline Sm-151 & $2.32 \mathrm{E}-01$ & $5.24 \mathrm{E}+01$ & 1.66E-02 & $5.27 \mathrm{E}+01$ & $3.27 \mathrm{E}-01$ & $5.24 \mathrm{E}+01$ & 4.14E-02 & $5.28 \mathrm{E}+01$ \\
\hline Sn-113 & $3.69 E+03$ & & $9.33 \mathrm{E}-01$ & $3.69 \mathrm{E}+03$ & $4.99 \mathrm{E}+03$ & & $2.33 E+00$ & $4.99 \mathrm{E}+03$ \\
\hline Sn-117m & 7.86E-06 & & 4.97E-06 & $1.28 \mathrm{E}-05$ & $2.08 \mathrm{E}-05$ & & $1.24 \mathrm{E}-05$ & 3.33E-05 \\
\hline Sn-119m & $3.68 \mathrm{E}+04$ & 2.10E-02 & $3.34 \mathrm{E}-01$ & $3.68 \mathrm{E}+04$ & $5.01 \mathrm{E}+04$ & $5.29 \mathrm{E}-02$ & 8.35E-01 & $5.01 \mathrm{E}+04$ \\
\hline Sn-121 & $5.76 \mathrm{E}+01$ & & & $5.76 \mathrm{E}+01$ & $1.05 \mathrm{E}+02$ & & & $1.05 E+02$ \\
\hline Sn-121m & $7.43 E+01$ & & & $7.43 E+01$ & $1.36 \mathrm{E}+02$ & & & $1.36 \mathrm{E}+02$ \\
\hline Sn-123 & $1.68 \mathrm{E}+01$ & $3.78 \mathrm{E}-03$ & $1.28 \mathrm{E}-02$ & $1.68 \mathrm{E}+01$ & $2.35 \mathrm{E}+01$ & $9.51 \mathrm{E}-03$ & 3.20E-02 & $2.35 \mathrm{E}+01$ \\
\hline Sn-125 & $5.78 \mathrm{E}-10$ & & & $5.78 \mathrm{E}-10$ & 1.77E-09 & & & 1.77E-09 \\
\hline Sn-126 & $1.29 \mathrm{E}-04$ & $5.04 \mathrm{E}-02$ & & $5.06 \mathrm{E}-02$ & $1.79 \mathrm{E}-04$ & $1.24 \mathrm{E}-01$ & & $1.24 \mathrm{E}-01$ \\
\hline Sr-85 & $2.52 \mathrm{E}-04$ & 1.93E-04 & & 4.45E-04 & $5.48 \mathrm{E}-04$ & 4.87E-04 & & $1.04 \mathrm{E}-03$ \\
\hline Sr-89 & $2.87 E+00$ & & $9.25 \mathrm{E}-06$ & $2.87 \mathrm{E}+00$ & $4.34 \mathrm{E}+00$ & & 2.31E-05 & $4.34 \mathrm{E}+00$ \\
\hline Sr-90 & $6.38 \mathrm{E}+00$ & $6.42 \mathrm{E}+02$ & $2.45 \mathrm{E}+01$ & $6.73 E+02$ & $9.31 \mathrm{E}+00$ & $6.45 \mathrm{E}+02$ & $6.13 \mathrm{E}+01$ & $7.15 E+02$ \\
\hline Ta-180 & $3.48 \mathrm{E}-01$ & & & $3.48 \mathrm{E}-01$ & $6.97 \mathrm{E}-01$ & & & 6.97E-01 \\
\hline Ta-182 & $4.61 \mathrm{E}+04$ & 1.70E-04 & $1.29 \mathrm{E}+00$ & $4.61 \mathrm{E}+04$ & $5.66 \mathrm{E}+04$ & 4.28E-04 & $3.23 E+00$ & $5.66 \mathrm{E}+04$ \\
\hline Tb-160 & $8.43 \mathrm{E}-05$ & & & 8.43E-05 & $2.35 \mathrm{E}-04$ & & & $2.35 \mathrm{E}-04$ \\
\hline Tc-98 & & $1.23 \mathrm{E}-07$ & & $1.23 \mathrm{E}-07$ & & $3.00 \mathrm{E}-07$ & & $3.00 \mathrm{E}-07$ \\
\hline Tc-99 & $9.85 \mathrm{E}-02$ & $3.11 \mathrm{E}+00$ & $2.03 E+00$ & $5.24 \mathrm{E}+00$ & $1.74 \mathrm{E}-01$ & $3.67 \mathrm{E}+00$ & $5.07 \mathrm{E}+00$ & $8.91 E+00$ \\
\hline Te-121 & $5.84 \mathrm{E}-03$ & & & $5.84 \mathrm{E}-03$ & 7.84E-03 & & & 7.84E-03 \\
\hline Te-121m & $5.88 \mathrm{E}-03$ & & & $5.88 \mathrm{E}-03$ & 7.89E-03 & & & 7.89E-03 \\
\hline Te-123 & 1.33E-09 & & & $1.33 \mathrm{E}-09$ & 4.95E-09 & & & 4.95E-09 \\
\hline Te-123m & $1.52 \mathrm{E}-02$ & & 1.23E-06 & $1.52 \mathrm{E}-02$ & $2.79 \mathrm{E}-02$ & & 3.08E-06 & 2.79E-02 \\
\hline Te-125m & $5.54 \mathrm{E}+03$ & $2.84 \mathrm{E}+00$ & $7.99 \mathrm{E}+00$ & $5.55 \mathrm{E}+03$ & $8.41 \mathrm{E}+03$ & $8.50 \mathrm{E}+00$ & $2.00 \mathrm{E}+01$ & $8.44 E+03$ \\
\hline Te-127 & 4.79E-01 & $1.10 \mathrm{E}-03$ & 5.84E-09 & 4.80E-01 & $6.28 \mathrm{E}-01$ & 2.77E-03 & 1.46E-08 & 6.31E-01 \\
\hline Te-127m & 4.89E-01 & $1.13 \mathrm{E}-03$ & 5.97E-09 & $4.90 \mathrm{E}-01$ & $6.42 \mathrm{E}-01$ & $2.85 \mathrm{E}-03$ & $1.49 \mathrm{E}-08$ & 6.44E-01 \\
\hline Te-129 & $9.30 \mathrm{E}-05$ & & & $9.30 \mathrm{E}-05$ & $2.34 \mathrm{E}-04$ & & & 2.34E-04 \\
\hline Te-129m & 1.46E-04 & & & $1.46 \mathrm{E}-04$ & 3.66E-04 & & & 3.66E-04 \\
\hline Te-132 & & & 7.94E-08 & 7.94E-08 & & & 1.99E-07 & 1.99E-07 \\
\hline Th-227 & $1.49 \mathrm{E}-06$ & & & 1.49E-06 & $1.81 \mathrm{E}-06$ & & & 1.81E-06 \\
\hline Th-228 & $2.01 \mathrm{E}-04$ & & $1.06 \mathrm{E}-06$ & 2.02E-04 & $2.84 \mathrm{E}-04$ & & 2.64E-06 & $2.86 \mathrm{E}-04$ \\
\hline Th-229 & 5.35E-08 & $1.75 \mathrm{E}-15$ & & $5.35 \mathrm{E}-08$ & $6.35 \mathrm{E}-08$ & $1.75 \mathrm{E}-15$ & & 6.35E-08 \\
\hline Th-230 & $4.81 \mathrm{E}-08$ & 1.26E-09 & & 4.93E-08 & $6.76 \mathrm{E}-08$ & 1.26E-09 & & $6.88 \mathrm{E}-08$ \\
\hline Th-231 & $2.97 \mathrm{E}-06$ & $4.78 \mathrm{E}-11$ & & 2.97E-06 & $8.02 \mathrm{E}-06$ & $1.20 \mathrm{E}-10$ & & 8.02E-06 \\
\hline Th-232 & $1.28 \mathrm{E}-07$ & $1.20 \mathrm{E}-07$ & & 2.48E-07 & $1.55 \mathrm{E}-07$ & $1.55 \mathrm{E}-07$ & & 3.10E-07 \\
\hline
\end{tabular}


TEM-10200-1

$03 / 01 / 2012$

ENGINEERING CALCULATIONS AND ANALYSIS

Page 28 of 30

Rev. 06

Baseline Radionuclide Inventory for the Remote-Handled Low-Level Waste Disposal

Title: $\quad$ Facility for Use in the Facility Performance Assessment

$\begin{array}{lllll}\text { ECAR No.: } 3940 \quad \text { Rev. No.: } & 0 & \text { Project No.: } & 31055 & \text { Date: } 01 / 24 / 18\end{array}$

Table 16. (continued).

\begin{tabular}{|c|c|c|c|c|c|c|c|c|}
\hline \multirow[b]{2}{*}{ Nuclide } & \multicolumn{4}{|c|}{ 20-Year Inventory } & \multicolumn{4}{|c|}{ 50-Year Inventory } \\
\hline & AM & SC & Resin & Total & AM & SC & Resin & Total \\
\hline Th-234 & $1.35 \mathrm{E}-04$ & & & $1.35 \mathrm{E}-04$ & $2.84 \mathrm{E}-04$ & & & 2.84E-04 \\
\hline TI-204 & 1.10E-22 & & & $1.10 \mathrm{E}-22$ & $2.19 E-22$ & & & 2.19E-22 \\
\hline TI-206 & $3.04 \mathrm{E}-03$ & & & $3.04 \mathrm{E}-03$ & $6.08 \mathrm{E}-03$ & & & $6.08 \mathrm{E}-03$ \\
\hline TI-207 & $1.51 \mathrm{E}-06$ & 4.17E-06 & & 5.69E-06 & $1.84 \mathrm{E}-06$ & $1.05 \mathrm{E}-05$ & & 1.24E-05 \\
\hline TI-208 & 7.26E-05 & $7.25 \mathrm{E}-05$ & & $1.45 E-04$ & $1.02 \mathrm{E}-04$ & $1.83 \mathrm{E}-04$ & & 2.85E-04 \\
\hline TI-209 & $8.68 \mathrm{E}-11$ & & & $8.68 \mathrm{E}-11$ & $2.89 \mathrm{E}-10$ & & & $2.89 \mathrm{E}-10$ \\
\hline $\mathrm{Tm}-170$ & $1.55 \mathrm{E}-11$ & & & $1.55 \mathrm{E}-11$ & $4.63 \mathrm{E}-11$ & & & 4.63E-11 \\
\hline Tm-171 & $2.55 \mathrm{E}-07$ & & & 2.55E-07 & $3.43 \mathrm{E}-07$ & & & 3.43E-07 \\
\hline $\mathrm{U}-232$ & $9.88 \mathrm{E}-05$ & $1.32 \mathrm{E}-04$ & & 2.31E-04 & $1.24 \mathrm{E}-04$ & $2.31 \mathrm{E}-04$ & & $3.55 \mathrm{E}-04$ \\
\hline U-233 & 7.91E-05 & $4.61 \mathrm{E}-06$ & & 8.38E-05 & $1.13 \mathrm{E}-04$ & $6.75 \mathrm{E}-06$ & & $1.20 \mathrm{E}-04$ \\
\hline U-234 & $1.20 \mathrm{E}-04$ & $1.25 \mathrm{E}-04$ & 3.64E-04 & 6.09E-04 & $2.58 \mathrm{E}-04$ & $2.66 \mathrm{E}-04$ & $9.11 \mathrm{E}-04$ & 1.44E-03 \\
\hline U-235 & 2.97E-06 & $3.70 \mathrm{E}-03$ & $8.18 \mathrm{E}-06$ & $3.71 E-03$ & 8.02E-06 & $3.70 \mathrm{E}-03$ & $2.05 E-05$ & 3.73E-03 \\
\hline U-236 & 1.47E-05 & $2.32 \mathrm{E}-06$ & 4.17E-05 & $5.88 \mathrm{E}-05$ & $2.25 \mathrm{E}-05$ & $5.07 \mathrm{E}-06$ & $1.04 \mathrm{E}-04$ & 1.32E-04 \\
\hline U-237 & $4.71 \mathrm{E}-04$ & & $1.03 \mathrm{E}-06$ & 4.72E-04 & $6.38 \mathrm{E}-04$ & & $2.58 \mathrm{E}-06$ & $6.40 \mathrm{E}-04$ \\
\hline U-238 & 1.35E-04 & 7.41E-04 & $1.69 \mathrm{E}-08$ & 8.76E-04 & $2.84 \mathrm{E}-04$ & 7.42E-04 & $4.22 \mathrm{E}-08$ & 1.03E-03 \\
\hline U-240 & $2.65 \mathrm{E}-13$ & & & $2.65 \mathrm{E}-13$ & 8.77E-13 & & & 8.77E-13 \\
\hline$V-49$ & & $2.10 \mathrm{E}-11$ & & $2.10 \mathrm{E}-11$ & & $2.10 \mathrm{E}-11$ & & $2.10 \mathrm{E}-11$ \\
\hline V-50 & $1.25 \mathrm{E}-11$ & & & $1.25 \mathrm{E}-11$ & $2.51 \mathrm{E}-11$ & & & 2.51E-11 \\
\hline W-181 & $4.29 \mathrm{E}+01$ & & & $4.29 E+01$ & $6.01 \mathrm{E}+01$ & & & $6.01 E+01$ \\
\hline W-185 & $2.32 \mathrm{E}+02$ & & & $2.32 \mathrm{E}+02$ & $3.19 \mathrm{E}+02$ & & & $3.19 \mathrm{E}+02$ \\
\hline W-187 & & & 3.58E-01 & 3.58E-01 & & & 8.94E-01 & 8.94E-01 \\
\hline W-188 & $1.25 \mathrm{E}-01$ & & & $1.25 \mathrm{E}-01$ & 1.87E-01 & & & 1.87E-01 \\
\hline$X e-131 m$ & $4.76 \mathrm{E}-11$ & & & $4.76 \mathrm{E}-11$ & $1.39 \mathrm{E}-10$ & & & 1.39E-10 \\
\hline Y-89m & 2.04E-04 & & & 2.04E-04 & 3.30E-04 & & & $3.30 \mathrm{E}-04$ \\
\hline Y-90 & $6.38 \mathrm{E}+00$ & $2.03 E+00$ & $2.33 E+01$ & $3.17 E+01$ & $9.31 \mathrm{E}+00$ & $5.06 \mathrm{E}+00$ & $5.81 \mathrm{E}+01$ & $7.25 E+01$ \\
\hline Y-91 & $1.48 \mathrm{E}+01$ & & 3.55E-05 & $1.48 \mathrm{E}+01$ & $2.25 \mathrm{E}+01$ & & 8.87E-05 & $2.25 \mathrm{E}+01$ \\
\hline Zn-65 & $1.76 \mathrm{E}+01$ & $9.90 \mathrm{E}+00$ & $3.25 \mathrm{E}+01$ & $6.00 \mathrm{E}+01$ & $2.73 E+01$ & $2.82 \mathrm{E}+01$ & $8.11 \mathrm{E}+01$ & $1.37 E+02$ \\
\hline Zr-93 & $2.21 \mathrm{E}+01$ & 1.09E-01 & 1.86E-02 & $2.22 \mathrm{E}+01$ & $4.29 \mathrm{E}+01$ & 1.66E-01 & 4.65E-02 & $4.31 \mathrm{E}+01$ \\
\hline Zr-95 & $3.59 \mathrm{E}+04$ & $1.98 \mathrm{E}+00$ & $1.57 \mathrm{E}+01$ & $3.59 \mathrm{E}+04$ & $5.01 \mathrm{E}+04$ & $5.42 \mathrm{E}+00$ & $3.93 \mathrm{E}+01$ & $5.02 \mathrm{E}+04$ \\
\hline Totals & $1.01 \mathrm{E}+06$ & $5.75 \mathrm{E}+03$ & $8.39 \mathrm{E}+03$ & $1.02 \mathrm{E}+06$ & $1.83 \mathrm{E}+06$ & $1.46 \mathrm{E}+04$ & $2.10 \mathrm{E}+04$ & $1.86 \mathrm{E}+06$ \\
\hline
\end{tabular}


TEM-10200-1

$03 / 01 / 2012$

ENGINEERING CALCULATIONS AND ANALYSIS

Page 29 of 30

Rev. 06

Title: $\quad$ Facility for Use in the Facility Performance Assessment

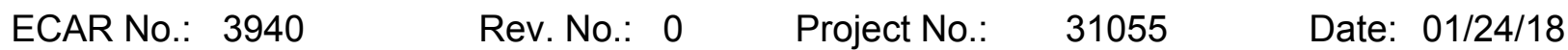

\section{REFERENCES}

DOE/ID-11421, 2012, "Performance Assessment for the Idaho National Laboratory Remote-Handled Low-Level Waste Disposal Facility," Revision 1, U.S. Department of Energy Idaho Operations Office, May 2012.

ECAR-851, 2011, "Estimate of the Radionuclide Content of Future Resins Generation at ATR from 2016 to 2065," Engineering Calculations and Analysis Report, Idaho National Laboratory, Revision 3, December 2011.

ECAR-854, 2010, "Estimate of the Radionuclide Content of Future Activated Metal Generation at ATR from 2016 to 2065," Engineering Calculations and Analysis Report, Revision 1, Idaho National Laboratory, December 2011.

ECAR-904, 2011, "A Methodology for Retrofitting Source-Terms to Previously Inadequately Characterized EBR-II Irradiated Reactor Hardware as Waste (Source Term and Volume Estimate for MFC Generated Remote Handled Low Level Waste from 2016 to 2065)," Engineering Calculations and Analysis Report, Revision 1, Idaho National Laboratory, December 2011.

ECAR-967, 2011, "Source Term and Volume Estimate for MFC Generated Remote Handled Low Level Waste from 2016 to 2065," Engineering Calculations and Analysis Report, Revision 1, Idaho National Laboratory, December 2011.

ECAR-1588, 2011, "RWSF Legacy Recasting Waste U and Pu Adjustments," Engineering Calculations and Analysis Report, Idaho National Laboratory, July 2011.

ECAR-3225, 2017, "Baseline Profile of the Remote-Handled Low-Level Waste Streams at the Materials and Fuels Complex," Revision 3, Idaho National Laboratory, December 2017.

ECAR-3340, 2016, "Detailed Configuration Analysis of the Remote-Handled Low-Level Waste Streams at the Materials and Fuels Complex," Idaho National Laboratory, August 2016.

EPA, 1999, Cancer Risk Coefficients for Environmental Exposure to Radionuclides, Federal Guidance Report No. 13, EPA 402-R-99-001, Office of Radiation and Indoor Air, U.S. Environmental Protection Agency, September 1999.

Jeff Frazier email to Jeff Sondrup, July 28, 2010, Subject: Re: Questions.

Jeff Frazier email to Brett Welty, September 20, 2017, Subject: Re: RH-LLW inventory questions and requests.

NRF, 2011, "Naval Nuclear Propulsion Program Remote-Handled Low-Level Radioactive Waste Generation Data," Letter from G. F. Holden, Manager, Naval Reactors, to R. Furstenau, DOE Idaho Operations Office, NR Letter U\#11-01756, April 15, 2011.

Stacy Nottestad email to Brett Welty, September 27, 2017, Subject: Re: RH-LLW inventory questions and requests. 
TEM-10200-1

$03 / 01 / 2012$

ENGINEERING CALCULATIONS AND ANALYSIS

Page 30 of 30

Rev. 06

Baseline Radionuclide Inventory for the Remote-Handled Low-Level Waste Disposal

Title: $\quad$ Facility for Use in the Facility Performance Assessment

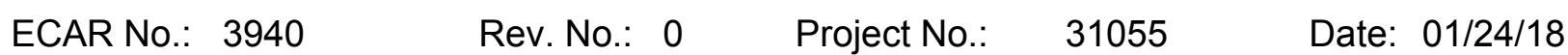

Stacy Nottestad email to Jeff Sondrup, October 31, 2017, Subject: Re: Question.

TEV-2921, 2017, "Delta Report: Comparison of Previous LLW Documents (ECAR-904, ECAR-967, ECAR 1558) and ECAR-3225," Technical Evaluation-2921, Idaho National Laboratory, March 2017. 
Rev. 06

Baseline Radionuclide Inventory for the Remote-Handled
Title: $\quad$ Facility for Use in the Facility Performance Assessment

ECAR No:: 3940

Rev. No.: 0

Project No.: 31055

Date: $01 / 24 / 18$

\section{Appendix A}

\section{DIF.FOR: A FORTRAN Program for Calculating Radioactive Decay and Ingrowth}

Radioactive decay and ingrowth is calculated using the Bateman equations. The generalized form of these equations is given by (Scrable et al. 1974; Whicker and Schultz 1982):

$$
q_{n}(t)=q_{1}\left[\left(\prod_{i=1}^{n} \lambda_{i}\right) \sum_{i=1}^{n}\left(\frac{e^{-\lambda_{i} t}}{\prod_{\substack{j \neq i \\ j=1}}^{n}\left(\lambda_{j}-\lambda_{i}\right)}\right)\right]
$$

where

$$
\begin{aligned}
& n=\text { the number in the decay chain series (i.e., } 1,2,3 \ldots n) \\
& q_{i}=\text { the number of atoms of progeny } i(i=1 \text { for parent) } \\
& q_{1}=\text { the number of parent atoms at } \mathrm{t}=0 . \\
& \lambda_{1}=\text { decay constant for the parent }\left(\mathrm{y}^{-1}\right) \\
& \lambda_{i}=\text { decay constant for the } \mathrm{i}^{\text {th }} \text { progeny }\left(\mathrm{y}^{-1}\right) \\
& t=\text { time from waste emplacement or release from the source (years). }
\end{aligned}
$$

Equation (1) is based on an initial condition of $t=0, q_{1}>0, q_{2}, q_{3} \ldots q_{n}=0$. Equation (1) is written in terms of the number of atoms. Activity is defined as

$$
A=\lambda N
$$

where $\mathrm{N}$ is the number of atoms. Thus, $\lambda q_{n}$ is the amount of progeny activity and $q_{1} / \lambda$ is the number of parent atoms at $t=0$. Substitution of (2) into (1) yields the activity of the progeny

$$
A_{n}(t)=\frac{q_{1} \lambda_{n}}{\lambda_{1}}\left[\left(\prod_{i=1}^{n} \lambda_{i}\right) \sum_{i=1}^{n}\left(\frac{e^{-\lambda_{i} t}}{\prod_{\substack{j \neq i \\ j=1}}^{n}\left(\lambda_{j}-\lambda_{i}\right)}\right)\right]
$$

The DIF.FOR program implements Equation 3 for a decay chain of $n$ members and calculates the amount of activity as a function of time for each member. Input to the code is through a single ASCII file termed the parameter definition file. The format of the parameter definition file is described in Table A-1. 
TEM-10200-1

$03 / 01 / 2012$

ENGINEERING CALCULATIONS AND ANALYSIS

Page $\mathrm{A} 2$ of $\mathrm{A} 11$

Rev. 06

Title: $\quad$ Facility for Use in the Facility Performance Assessment

$\begin{array}{llll}\text { ECAR No.: } 3940 \quad \text { Rev. No.: } & 0 & \text { Project No.: } 31055 & \text { Date: } 01 / 24 / 18\end{array}$

As stated in the main body of this ECAR, decay chains were shortened to include only long-lived members. Short-lived members that would generally be in secular equilibrium with their parent within about a year were not explicitly included. For example, the short-lived progeny of Ra-226 include Rn-222, Po-218, Pb-214, Bi-214, and Po-214. Rn-222 has the longest half-life (3.82 days) and within 38 days would generally be in secular equilibrium with Ra-226 (assuming radon stays in place and does not diffuse out of the waste matrix). Thus, these progeny were not modeled explicitly and only the next long-lived progeny (Pb-210, half-life of 22 years) was included in the decay chain.

Table A-1. Format for the DIF program parameter definition file (*.par).

\begin{tabular}{|c|c|c|c|}
\hline Record & $\begin{array}{c}\text { Code } \\
\text { Variable }\end{array}$ & Type/Format & Description \\
\hline 1 & $\mathrm{nbr}$ & INT/* & Number of decay chain members \\
\hline 1 & $\mathrm{a} 0$ & $\mathrm{REAL} /{ }^{*}$ & Initial parent activity \\
\hline 1 & units & $\mathrm{CHAR} /{ }^{*}$ & Units of activity ( $\mathrm{Ci}$ or $\mathrm{Bq})$ \\
\hline 2 & nucname() & $\mathrm{CHAR} /{ }^{*}$ & Name of each decay chain member \\
\hline 3 & thalf() & $\mathrm{REAL} /{ }^{*}$ & Half-lives for each decay chain member (years) \\
\hline 4 & amass() & $\mathrm{REAL} / *$ & Atomic weight for each decay chain member $\left(\mathrm{g} \mathrm{mol}^{-1}\right)$ \\
\hline 5 & ntimes & INT/* & Number of output time blocks $($ maximum $=10)$ \\
\hline \multicolumn{4}{|c|}{ Repeat record 6 ntimes } \\
\hline 6 & $\mathrm{t} 1()$ & $\mathrm{REAL} /{ }^{*}$ & Begin time (years) \\
\hline 6 & $\mathrm{t} 2()$ & $\mathrm{REAL} / *$ & End time (years) \\
\hline 6 & $\operatorname{tp}()$ & $\mathrm{REAL} /{ }^{*}$ & Print step (years) \\
\hline
\end{tabular}

The computer code is run using an initial parent activity of $1 \mathrm{Ci}$. Thus the activity of each progeny must be multiplied by the actual activity of the parent to obtain the activity of the progeny.

\section{References}

Scrable, K. W., C. S. French, G. Chabot, and A. Major, 1974, "A General Equation for the Kinetics of Linear First Order Phenomena and Suggested Applications," Health Physics, 27: pp. 155-157.

Whicker, F. W. and V. Schultz, 1982, Radioecology: Nuclear Energy and the Environment, CRC Press, Boca Raton, Florida. 
TEM-10200-1

$03 / 01 / 2012$

ENGINEERING CALCULATIONS AND ANALYSIS

Page $A 3$ of $A 11$

Rev. 06

Title: $\quad$ Facility for Use in the Facility Performance Assessment

$\begin{array}{llll}\text { ECAR No.: } 3940 \quad \text { Rev. No.: } & 0 & \text { Project No.: } 31055 & \text { Date: } 01 / 24 / 18\end{array}$

program dif

\section{DIF.FOR Program Listing}

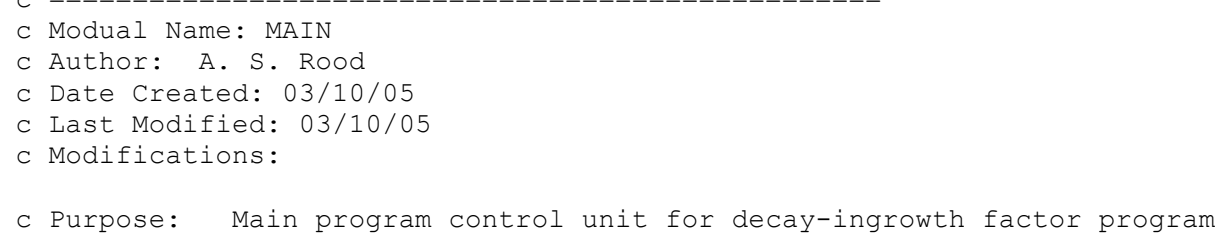


Rev. 06

Baseline Radionuclide Inventory for the Remote-Handled Low-Level Waste Disposal

Title: $\quad$ Facility for Use in the Facility Performance Assessment

ECAR No.: 3940

Rev. No.: 0

Project No.: 31055

Date: $01 / 24 / 18$

close (1)

c calculate lambda
do $i=1$, nbr
lambda(i)= $\log (2$.$) / thalf (i)$
enddo

c calculate specific activity and initial number of atoms

if (units.eq.'Ci') then

$\mathrm{q} 0=\left(\mathrm{a} 0{ }^{*} \mathrm{ci}\right) /($ lambda $(1) / \mathrm{sec})$

do $i=1, n b r$

$\operatorname{SpA}(i)=1.0 /(\mathrm{ci} /($ lambda $(i) / \sec ) *(1.0 / \operatorname{agn}) * \operatorname{amass}(i))$ enddo

endif

if (units.eq. 'Bq') then

q0 $=\mathrm{a} 0 /($ lambda $(1) / \mathrm{sec})$

do $i=1$, nbr

$\operatorname{SpA}(i)=1.0 /(1.0 /($ lambda $(i) / \mathrm{sec}) *(1 / \operatorname{agn}) * \operatorname{amass}(i))$ enddo

endif

write $(*, *)$ 'Activity in ', units

write (*, ' (a11, $30(2 \mathrm{x}, \mathrm{a} 6,3 \mathrm{x}))$ ') 'Parameter ', (nucname (i), i=1, nbr)

write (*,' (a11,30(1pe10.3,1x)) ') 'Lambda(1/y)',

\& (lambda (i), $i=1, \mathrm{nbr}$ )

write (*,' (a4,a2, a2,3x, $30(1 \mathrm{pe} 10.3,1 \mathrm{x}))$ ') 'SpA ', units, '/g',

$\& \quad(\operatorname{SpA}(i), i=1, \mathrm{nbr})$

write (*, ' (a11,30 (2x,a6,3x))') 'Time (yr) ', (nucname(i), i=1, nbr)

c calculate activities

do $i=1$, ntimes

nstep=int ( (t2 (i) -t1 (i))/tp (i))

$t=t 1$ (i)

do $j=1$, nstep

$\mathrm{a}(1)=\mathrm{a} 0 * \exp (-1 \mathrm{ambda}(1) * t)$

do $k=2$, nbr

call difcalc $(k, l a m b d a, t, q, q 0)$

$\mathrm{a}(\mathrm{k})=\mathrm{q}^{\star}$ lambda $(\mathrm{k}) / \mathrm{sec}$

if (units.eq. 'Ci') then $\mathrm{a}(\mathrm{k})=\mathrm{a}(\mathrm{k}) / \mathrm{ci}$

endif

enddo

write (*,' (11(1pe11.4))') t, (a (k), k=1, nbr)

$t=t+\operatorname{tp}(i)$

enddo

enddo

end

subroutine difcalc(nbr, lambda, t, $q, q 0)$

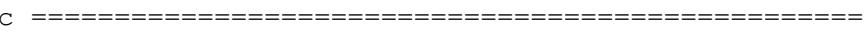

C Modual Name: DIFCALC

C Author: A. S. Rood

c Date Created: 03/10/05

c Last Modified: 03/10/05

c Modifications:

c Purpose: calculates number of atoms in decay chain member nbr at time $t$

c givin an initial amount q0 at time zero

c Arguments:

c nbr: num

or passes it back to the calling rountine

nbr: number of decay chain members

lambda: decay rate constant $(y-1)$

$t: \quad$ time (years) 
TEM-10200-1

$03 / 01 / 2012$

ENGINEERING CALCULATIONS AND ANALYSIS

Page A5 of $\mathrm{A} 11$

Rev. 06

Baseline Radionuclide Inventory for the Remote-Handled Low-Level Waste Disposal

Title: $\quad$ Facility for Use in the Facility Performance Assessment

ECAR No.: 3940

Rev. No.: 0

Project No.: 31055

Date: $01 / 24 / 18$

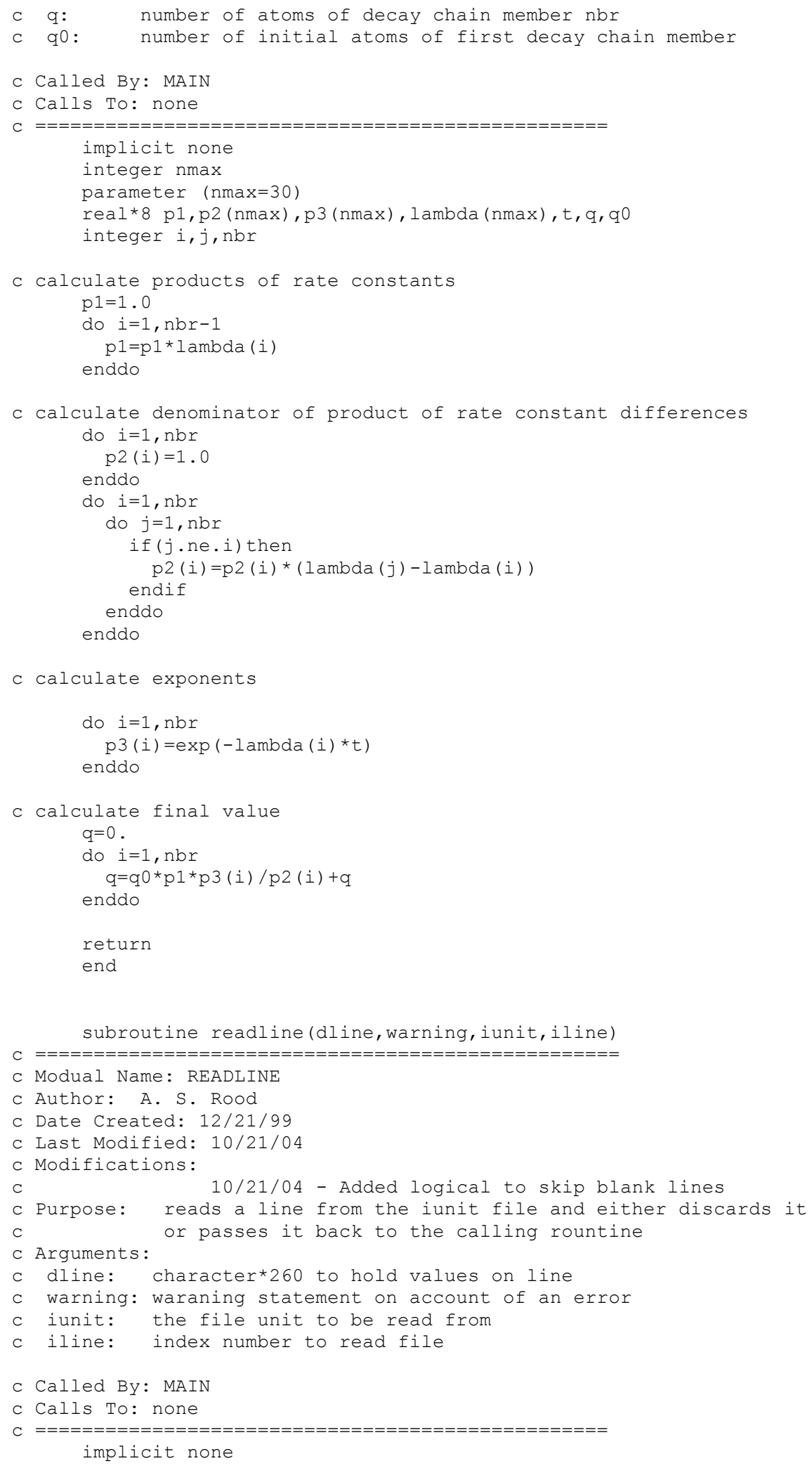


Rev. 06

\section{Baseline Radionuclide Inventory for the Remote-Handled Low-Level Waste Disposal}

Title: $\quad$ Facility for Use in the Facility Performance Assessment

ECAR No.: $3940 \quad$ Rev. No.: $0 \quad$ Project No.: 31055

Date: $01 / 24 / 18$

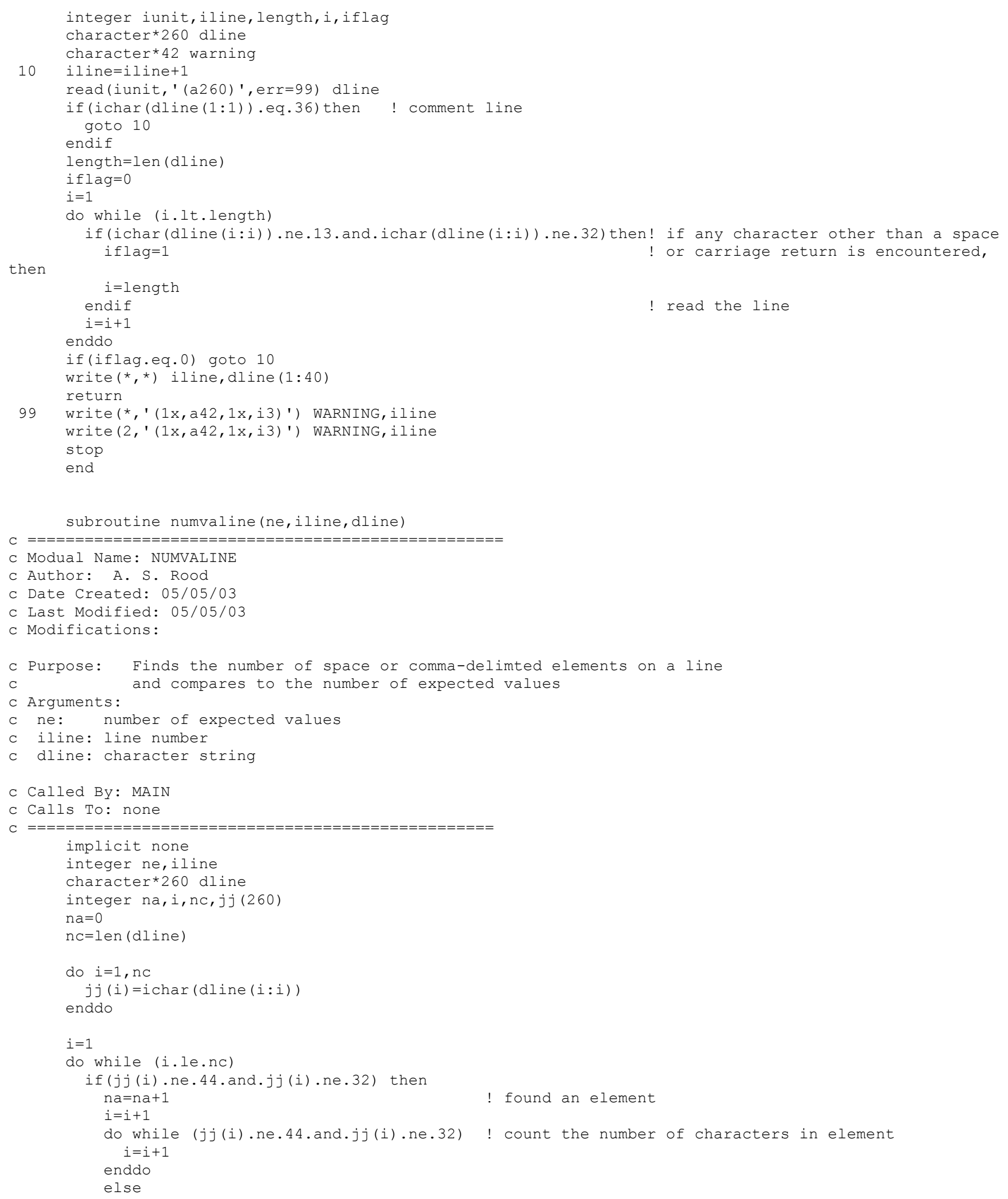


TEM-10200-1

$03 / 01 / 2012$

ENGINEERING CALCULATIONS AND ANALYSIS

Page $A 7$ of $A 11$

Rev. 06

Baseline Radionuclide Inventory for the Remote-Handled Low-Level Waste Disposal

Title: $\quad$ Facility for Use in the Facility Performance Assessment

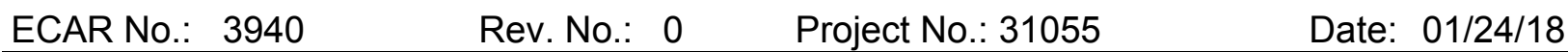

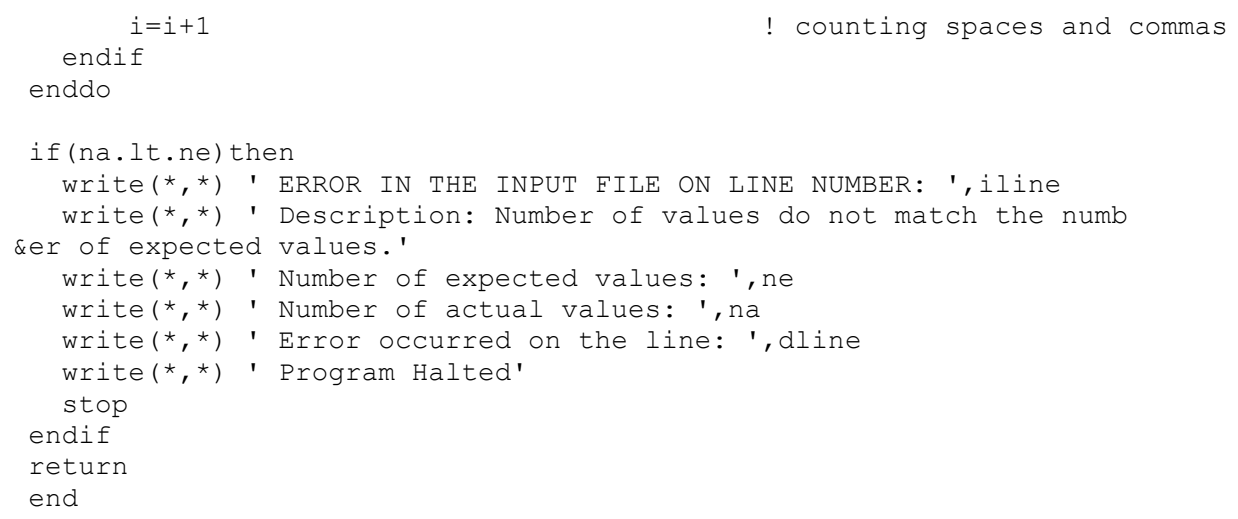


TEM-10200-1

$03 / 01 / 2012$

ENGINEERING CALCULATIONS AND ANALYSIS

Page $A 8$ of $A 11$

Rev. 06

Baseline Radionuclide Inventory for the Remote-Handled Low-Level Waste Disposal

Title: $\quad$ Facility for Use in the Facility Performance Assessment

$\begin{array}{llll}\text { ECAR No.: } 3940 \quad \text { Rev. No.: } & 0 & \text { Project No.: } 31055 & \text { Date: } 01 / 24 / 18\end{array}$

\section{Input Files (*.par)}

Although files are named *.par, the names must be changed to dif.par to be read by the program. The output times are 1 to 50 years by 1 year. This is sufficient to decay the legacy inventory.

\section{Am-241.par}

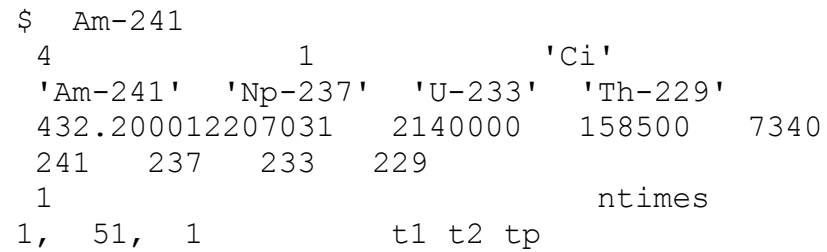

\section{Pu-238.par}

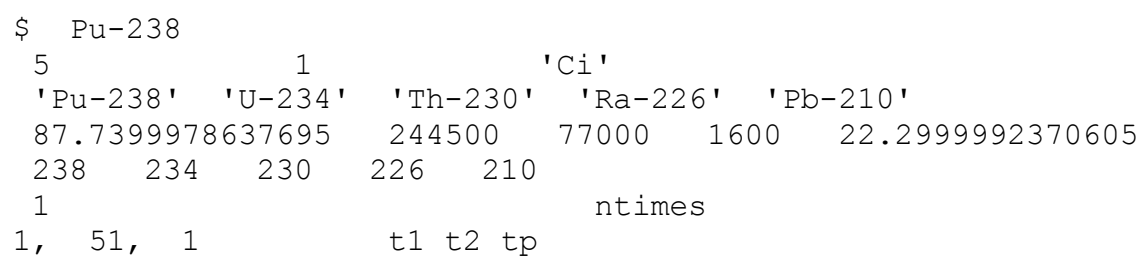

\section{Pu-241.par}

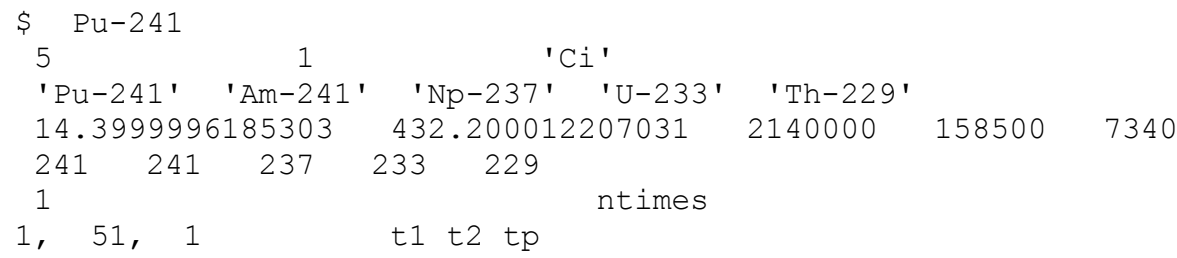

\section{Output Files ( ${ }^{*}$. Ist)}

$\underline{\text { Am-241.Ist }}$

\begin{tabular}{|c|c|c|c|c|c|}
\hline & 4 & 1 & 'Ci & $i^{\prime}$ & \\
\hline & 'Am-241' & 'Np-237' & 'U-233' & 'Th-229' & \\
\hline & 432.2000 & 12207031 & 2140000 & 158500 & 7 \\
\hline & 241 & 232 & 229 & & \\
\hline & 1 & & & ntimes & \\
\hline & 81, & 1 & t1 t2 tp & & \\
\hline Act & $\mathrm{n} \mathrm{Ci}$ & & & & \\
\hline neter & $A m-241$ & $N p-237$ & $\mathrm{U}-233$ & Th-22 & \\
\hline Lambda (1/y) & $1.604 \mathrm{E}-03$ & $3.239 \mathrm{E}-07$ & $4.373 E-06$ & 9.4 & -05 \\
\hline SpA Ci/g & $3.433 \mathrm{E}+00$ & $7.050 \mathrm{E}-04$ & $9.682 E-03$ & 2.1 & -01 \\
\hline$(y r)$ & $A m-241$ & $N p-237$ & $\mathrm{U}-$ & Th & \\
\hline $1.0000 \mathrm{E}+00$ & $9.9840 \mathrm{E}-01$ & $3.2364 \mathrm{E}-07$ & $6 E-13$ & 32.2 & $E-17$ \\
\hline $2.0000 \mathrm{E}+00$ & $9.9680 \mathrm{E}-01$ & $6.4676 \mathrm{E}-07$ & $7 \quad 2.8299 \mathrm{E}-12$ & 21.7817 & $E-16$ \\
\hline $3.0000 \mathrm{E}+00$ & $9.9520 E-01$ & $9.6937 E-07$ & $76.3639 \mathrm{E}-12$ & 26.0115 & $E-16$ \\
\hline
\end{tabular}


Rev. 06

Title: $\quad$ Facility for Use in the Facility Performance Assessment

$\begin{array}{llll}\text { ECAR No.: } 3940 \quad \text { Rev. No.: } & 0 & \text { Project No.: } 31055 \quad \text { Date: } 01 / 24 / 18\end{array}$

$\begin{array}{llllll}4.0000 \mathrm{E}+00 & 9.9361 \mathrm{E}-01 & 1.2915 \mathrm{E}-06 & 1.1308 \mathrm{E}-11 & 1.4244 \mathrm{E}-15\end{array}$

$\begin{array}{llllll}5.0000 \mathrm{E}+00 & 9.9201 \mathrm{E}-01 & 1.6130 \mathrm{E}-06 & 1.7659 \mathrm{E}-11 & 2.7808 \mathrm{E}-15\end{array}$

$6.0000 \mathrm{E}+00 \quad 9.9042 \mathrm{E}-01 \quad 1.9341 \mathrm{E}-06 \quad 2.5415 \mathrm{E}-11 \quad 4.8032 \mathrm{E}-15$

$\begin{array}{lllll}7.0000 \mathrm{E}+00 & 9.8884 \mathrm{E}-01 & 2.2546 \mathrm{E}-06 & 3.4574 \mathrm{E}-11 & 7.6240 \mathrm{E}-15\end{array}$

$\begin{array}{lllll}8.0000 \mathrm{E}+00 & 9.8725 \mathrm{E}-01 & 2.5746 \mathrm{E}-06 & 4.5133 \mathrm{E}-11 & 1.1376 \mathrm{E}-14\end{array}$

$9.0000 \mathrm{E}+00 \quad 9.8567 \mathrm{E}-01 \quad 2.8942 \mathrm{E}-06 \quad 5.7091 \mathrm{E}-11 \quad 1.6190 \mathrm{E}-14$

$\begin{array}{lllll}1.0000 \mathrm{E}+01 & 9.8409 \mathrm{E}-01 & 3.2132 \mathrm{E}-06 & 7.0445 \mathrm{E}-11 & 2.2199 \mathrm{E}-14\end{array}$

$\begin{array}{llllll}1.1000 \mathrm{E}+01 & 9.8251 \mathrm{E}-01 & 3.5317 \mathrm{E}-06 & 8.5193 \mathrm{E}-11 & 2.9535 \mathrm{E}-14\end{array}$

$\begin{array}{llllll}1.2000 \mathrm{E}+01 & 9.8094 \mathrm{E}-01 & 3.8496 \mathrm{E}-06 & 1.0133 \mathrm{E}-10 & 3.8328 \mathrm{E}-14\end{array}$

$\begin{array}{lllll}1.3000 \mathrm{E}+01 & 9.7937 \mathrm{E}-01 & 4.1671 \mathrm{E}-06 & 1.1886 \mathrm{E}-10 & 4.8710 \mathrm{E}-14\end{array}$

$\begin{array}{lllll}1.4000 \mathrm{E}+01 & 9.7780 \mathrm{E}-01 & 4.4841 \mathrm{E}-06 & 1.3778 \mathrm{E}-10 & 6.0811 \mathrm{E}-14\end{array}$

$1.5000 \mathrm{E}+01 \quad 9.7623 \mathrm{E}-01 \quad 4.8005 \mathrm{E}-06 \quad 1.5808 \mathrm{E}-10 \quad 7.4764 \mathrm{E}-14$

$\begin{array}{llllll}1.6000 \mathrm{E}+01 & 9.7467 \mathrm{E}-01 & 5.1165 \mathrm{E}-06 & 1.7976 \mathrm{E}-10 & 9.0697 \mathrm{E}-14\end{array}$

$\begin{array}{lllll}1.7000 \mathrm{E}+01 & 9.7310 \mathrm{E}-01 & 5.4319 \mathrm{E}-06 & 2.0283 \mathrm{E}-10 & 1.0874 \mathrm{E}-13\end{array}$

$\begin{array}{lllll}1.8000 \mathrm{E}+01 & 9.7154 \mathrm{E}-01 & 5.7468 \mathrm{E}-06 & 2.2727 \mathrm{E}-10 & 1.2903 \mathrm{E}-13\end{array}$

$\begin{array}{llllll}1.9000 \mathrm{E}+01 & 9.6999 \mathrm{E}-01 & 6.0613 \mathrm{E}-06 & 2.5309 \mathrm{E}-10 & 1.5168 \mathrm{E}-13\end{array}$

$2.0000 \mathrm{E}+01 \quad 9.6843 \mathrm{E}-01 \quad 6.3752 \mathrm{E}-06 \quad 2.8028 \mathrm{E}-10 \quad 1.7684 \mathrm{E}-13$

$\begin{array}{lllll}2.1000 \mathrm{E}+01 & 9.6688 \mathrm{E}-01 & 6.6886 \mathrm{E}-06 & 3.0884 \mathrm{E}-10 & 2.0463 \mathrm{E}-13\end{array}$

$\begin{array}{llllll}2.2000 \mathrm{E}+01 & 9.6533 \mathrm{E}-01 & 7.0015 \mathrm{E}-06 & 3.3878 \mathrm{E}-10 & 2.3518 \mathrm{E}-13\end{array}$

$\begin{array}{lllll}2.3000 \mathrm{E}+01 & 9.6379 \mathrm{E}-01 & 7.3140 \mathrm{E}-06 & 3.7008 \mathrm{E}-10 & 2.6861 \mathrm{E}-13\end{array}$

$2.4000 \mathrm{E}+01 \quad 9.6224 \mathrm{E}-01 \quad 7.6259 \mathrm{E}-06 \quad 4.0274 \mathrm{E}-10 \quad 3.0507 \mathrm{E}-13$

$2.5000 \mathrm{E}+01 \quad 9.6070 \mathrm{E}-01 \quad 7.9373 \mathrm{E}-06 \quad 4.3677 \mathrm{E}-10 \quad 3.4466 \mathrm{E}-13$

$\begin{array}{llllll}2.6000 \mathrm{E}+01 & 9.5916 \mathrm{E}-01 & 8.2482 \mathrm{E}-06 & 4.7216 \mathrm{E}-10 & 3.8754 \mathrm{E}-13\end{array}$

$\begin{array}{llllll}2.7000 \mathrm{E}+01 & 9.5762 \mathrm{E}-01 & 8.5586 \mathrm{E}-06 & 5.0891 \mathrm{E}-10 & 4.3381 \mathrm{E}-13\end{array}$

$2.8000 \mathrm{E}+01 \quad 9.5609 \mathrm{E}-01 \quad 8.8686 \mathrm{E}-06 \quad 5.4701 \mathrm{E}-10 \quad 4.8361 \mathrm{E}-13$

$\begin{array}{llllll}2.9000 \mathrm{E}+01 & 9.5456 \mathrm{E}-01 & 9.1780 \mathrm{E}-06 & 5.8647 \mathrm{E}-10 & 5.3708 \mathrm{E}-13\end{array}$

$\begin{array}{lllll}3.0000 \mathrm{E}+01 & 9.5303 \mathrm{E}-01 & 9.4869 \mathrm{E}-06 & 6.2728 \mathrm{E}-10 & 5.9432 \mathrm{E}-13\end{array}$

$\begin{array}{lllll}3.1000 \mathrm{E}+01 & 9.5150 \mathrm{E}-01 & 9.7954 \mathrm{E}-06 & 6.6944 \mathrm{E}-10 & 6.5548 \mathrm{E}-13\end{array}$

$3.2000 \mathrm{E}+01 \quad 9.4997 \mathrm{E}-01 \quad 1.0103 \mathrm{E}-05 \quad 7.1295 \mathrm{E}-10 \quad 7.2068 \mathrm{E}-13$

$3.3000 \mathrm{E}+01 \quad 9.4845 \mathrm{E}-01 \quad 1.0411 \mathrm{E}-05 \quad 7.5780 \mathrm{E}-10 \quad 7.9004 \mathrm{E}-13$

$3.4000 \mathrm{E}+01 \quad 9.4693 \mathrm{E}-01 \quad 1.0718 \mathrm{E}-05 \quad 8.0400 \mathrm{E}-10 \quad 8.6369 \mathrm{E}-13$

$\begin{array}{llllll}3.5000 \mathrm{E}+01 & 9.4541 \mathrm{E}-01 & 1.1024 \mathrm{E}-05 & 8.5153 \mathrm{E}-10 & 9.4177 \mathrm{E}-13\end{array}$

$\begin{array}{lllll}3.6000 \mathrm{E}+01 & 9.4390 \mathrm{E}-01 & 1.1330 \mathrm{E}-05 & 9.0041 \mathrm{E}-10 & 1.0244 \mathrm{E}-12\end{array}$

$3.7000 \mathrm{E}+01 \quad 9.4239 \mathrm{E}-01 \quad 1.1636 \mathrm{E}-05 \quad 9.5062 \mathrm{E}-10 \quad 1.1117 \mathrm{E}-12$

$\begin{array}{lllll}3.8000 \mathrm{E}+01 & 9.4088 \mathrm{E}-01 & 1.1941 \mathrm{E}-05 & 1.0022 \mathrm{E}-09 & 1.2038 \mathrm{E}-12\end{array}$

$3.9000 \mathrm{E}+01 \quad 9.3937 \mathrm{E}-01 \quad 1.2245 \mathrm{E}-05 \quad 1.0550 \mathrm{E}-09 \quad 1.3008 \mathrm{E}-12$

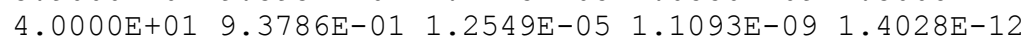
$\begin{array}{lllll}4.1000 \mathrm{E}+01 & 9.3636 \mathrm{E}-01 & 1.2853 \mathrm{E}-05 & 1.1648 \mathrm{E}-09 & 1.5100 \mathrm{E}-12\end{array}$ $\begin{array}{lllll}4.2000 \mathrm{E}+01 & 9.3486 \mathrm{E}-01 & 1.3156 \mathrm{E}-05 & 1.2217 \mathrm{E}-09 & 1.6226 \mathrm{E}-12\end{array}$ $\begin{array}{lllll}4.3000 \mathrm{E}+01 & 9.3336 \mathrm{E}-01 & 1.3458 \mathrm{E}-05 & 1.2799 \mathrm{E}-09 & 1.7405 \mathrm{E}-12\end{array}$ $\begin{array}{lllll}4.4000 \mathrm{E}+01 & 9.3187 \mathrm{E}-01 & 1.3760 \mathrm{E}-05 & 1.3394 \mathrm{E}-09 & 1.8640 \mathrm{E}-12\end{array}$ $4.5000 \mathrm{E}+01 \quad 9.3037 \mathrm{E}-01 \quad 1.4062 \mathrm{E}-05 \quad 1.4002 \mathrm{E}-09 \quad 1.9932 \mathrm{E}-12$ $\begin{array}{lllll}4.6000 \mathrm{E}+01 & 9.2888 \mathrm{E}-01 & 1.4363 \mathrm{E}-05 & 1.4623 \mathrm{E}-09 & 2.1281 \mathrm{E}-12\end{array}$ $\begin{array}{lllll}4.7000 \mathrm{E}+01 & 9.2739 \mathrm{E}-01 & 1.4664 \mathrm{E}-05 & 1.5258 \mathrm{E}-09 & 2.2690 \mathrm{E}-12\end{array}$ $\begin{array}{lllll}4.8000 \mathrm{E}+01 & 9.2591 \mathrm{E}-01 & 1.4964 \mathrm{E}-05 & 1.5906 \mathrm{E}-09 & 2.4159 \mathrm{E}-12\end{array}$ $\begin{array}{lllll}4.9000 \mathrm{E}+01 & 9.2442 \mathrm{E}-01 & 1.5263 \mathrm{E}-05 & 1.6567 \mathrm{E}-09 & 2.5690 \mathrm{E}-12\end{array}$ $\begin{array}{lllll}5.0000 \mathrm{E}+01 & 9.2294 \mathrm{E}-01 & 1.5563 \mathrm{E}-05 & 1.7241 \mathrm{E}-09 & 2.7284 \mathrm{E}-12\end{array}$

\section{$\underline{\text { Pu-238.Ist }}$}

Activity in $\mathrm{Ci}$

\begin{tabular}{|c|c|c|c|}
\hline 5 & 1 & & Ci' \\
\hline ' $\mathrm{Pu}-238$ ' & 'U-234' & ' $T h-230$ ' & 'Ra-226' \\
\hline 87.7399978 & 3637695 & 244500 & 77000 \\
\hline 234 & 230 & 226 & \\
\hline & & & ntimes \\
\hline 61, & & t1 t2 tp & \\
\hline
\end{tabular}

Parameter

$\mathrm{Pu}-238 \quad \mathrm{U}-234$

Lambda $(1 / y) \quad 7.900 \mathrm{E}-03 \quad 2.835 \mathrm{E}-06$

Th-230

$\mathrm{Ra}-226$

$\mathrm{Pb}-210$

9.002E-06 4.332E-04 3.108E-02 
Rev. 06

Title: $\quad$ Facility for Use in the Facility Performance Assessment

$\begin{array}{llll}\text { ECAR No.: } 3940 & \text { Rev. No.: } 0 & \text { Project No.: } 31055 & \text { Date: } 01 / 24 / 18\end{array}$

$\begin{array}{cccccc}\text { SpA Ci/g } & 1.712 \mathrm{E}+01 & 6.249 \mathrm{E}-03 & 2.019 \mathrm{E}-02 & 9.888 \mathrm{E}-01 & 7.635 \mathrm{E}+01 \\ \text { Time (yr) } & \text { Pu-238 } & \mathrm{U}-234 & \text { Th-230 } & \text { Ra-226 } & \text { Pb-210 }\end{array}$

$\begin{array}{lllllll}1.0000 \mathrm{E}+00 & 9.9213 \mathrm{E}-01 & 2.8238 \mathrm{E}-06 & 1.2726 \mathrm{E}-11 & 1.8388 \mathrm{E}-15 & 1.4284 \mathrm{E}-17\end{array}$

$\begin{array}{lllllll}2.0000 \mathrm{E}+00 & 9.8432 \mathrm{E}-01 & 5.6253 \mathrm{E}-06 & 5.0772 \mathrm{E}-11 & 1.4680 \mathrm{E}-14 & 2.2568 \mathrm{E}-16\end{array}$

$\begin{array}{lllllll}3.0000 \mathrm{E}+00 & 9.7658 \mathrm{E}-01 & 8.4048 \mathrm{E}-06 & 1.1394 \mathrm{E}-10 & 4.9441 \mathrm{E}-14 & 1.1328 \mathrm{E}-15\end{array}$

$\begin{array}{llllll}4.0000 \mathrm{E}+00 & 9.6889 \mathrm{E}-01 & 1.1162 \mathrm{E}-05 & 2.0202 \mathrm{E}-10 & 1.1695 \mathrm{E}-13 & 3.5524 \mathrm{E}-15\end{array}$

$\begin{array}{llllll}5.0000 \mathrm{E}+00 & 9.6127 \mathrm{E}-01 & 1.3898 \mathrm{E}-05 & 3.1484 \mathrm{E}-10 & 2.2794 \mathrm{E}-13 & 8.6057 \mathrm{E}-15\end{array}$

$\begin{array}{llllll}6.0000 \mathrm{E}+00 & 9.5371 \mathrm{E}-01 & 1.6613 \mathrm{E}-05 & 4.5218 \mathrm{E}-10 & 3.9307 \mathrm{E}-13 & 1.7707 \mathrm{E}-14\end{array}$

$\begin{array}{llllll}7.0000 \mathrm{E}+00 & 9.4620 \mathrm{E}-01 & 1.9306 \mathrm{E}-05 & 6.1386 \mathrm{E}-10 & 6.2289 \mathrm{E}-13 & 3.2551 \mathrm{E}-14\end{array}$

$\begin{array}{llllll}8.0000 \mathrm{E}+00 & 9.3876 \mathrm{E}-01 & 2.1978 \mathrm{E}-05 & 7.9968 \mathrm{E}-10 & 9.2787 \mathrm{E}-13 & 5.5105 \mathrm{E}-14\end{array}$

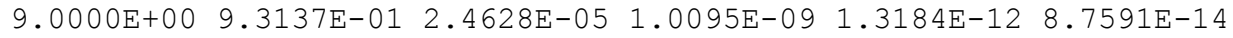

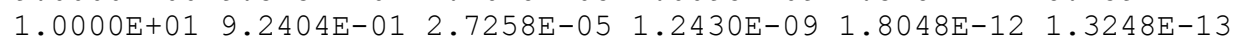

$\begin{array}{llllll}1.1000 \mathrm{E}+01 & 9.1677 \mathrm{E}-01 & 2.9868 \mathrm{E}-05 & 1.5001 \mathrm{E}-09 & 2.3972 \mathrm{E}-12 & 1.9249 \mathrm{E}-13\end{array}$

$\begin{array}{llllll}1.2000 \mathrm{E}+01 & 9.0955 \mathrm{E}-01 & 3.2456 \mathrm{E}-05 & 1.7806 \mathrm{E}-09 & 3.1058 \mathrm{E}-12 & 2.7056 \mathrm{E}-13\end{array}$

$\begin{array}{llllll}1.3000 \mathrm{E}+01 & 9.0240 \mathrm{E}-01 & 3.5024 \mathrm{E}-05 & 2.0844 \mathrm{E}-09 & 3.9407 \mathrm{E}-12 & 3.6984 \mathrm{E}-13\end{array}$

$\begin{array}{llllll}1.4000 \mathrm{E}+01 & 8.9530 \mathrm{E}-01 & 3.7573 \mathrm{E}-05 & 2.4111 \mathrm{E}-09 & 4.9117 \mathrm{E}-12 & 4.9370 \mathrm{E}-13\end{array}$

$\begin{array}{llllll}1.5000 \mathrm{E}+01 & 8.8825 \mathrm{E}-01 & 4.0101 \mathrm{E}-05 & 2.7607 \mathrm{E}-09 & 6.0288 \mathrm{E}-12 & 6.4571 \mathrm{E}-13\end{array}$

$\begin{array}{llllll}1.6000 \mathrm{E}+01 & 8.8126 \mathrm{E}-01 & 4.2609 \mathrm{E}-05 & 3.1330 \mathrm{E}-09 & 7.3017 \mathrm{E}-12 & 8.2962 \mathrm{E}-13\end{array}$

$\begin{array}{lllllll}1.7000 \mathrm{E}+01 & 8.7433 \mathrm{E}-01 & 4.5097 \mathrm{E}-05 & 3.5277 \mathrm{E}-09 & 8.7402 \mathrm{E}-12 & 1.0494 \mathrm{E}-12\end{array}$

$1.8000 \mathrm{E}+01 \quad 8.6745 \mathrm{E}-01 \quad 4.7566 \mathrm{E}-05 \quad 3.9448 \mathrm{E}-09 \quad 1.0354 \mathrm{E}-11 \quad 1.3091 \mathrm{E}-12$

$1.9000 \mathrm{E}+01 \quad 8.6062 \mathrm{E}-01 \quad 5.0015 \mathrm{E}-05 \quad 4.3840 \mathrm{E}-09 \quad 1.2152 \mathrm{E}-11 \quad 1.6131 \mathrm{E}-12$

$2.0000 \mathrm{E}+01$ 8.5385E-01 5.2445E-05 4.8451E-09 $1.4145 \mathrm{E}-11 \quad 1.9658 \mathrm{E}-12$

$\begin{array}{lllllll}2.1000 \mathrm{E}+01 & 8.4713 \mathrm{E}-01 & 5.4856 \mathrm{E}-05 & 5.3280 \mathrm{E}-09 & 1.6341 \mathrm{E}-11 & 2.3718 \mathrm{E}-12\end{array}$

$2.2000 \mathrm{E}+01 \quad 8.4046 \mathrm{E}-01 \quad 5.7248 \mathrm{E}-05 \quad 5.8326 \mathrm{E}-09 \quad 1.8750 \mathrm{E}-112.8358 \mathrm{E}-12$

$2.3000 \mathrm{E}+01 \quad 8.3385 \mathrm{E}-01 \quad 5.9621 \mathrm{E}-05 \quad 6.3586 \mathrm{E}-09 \quad 2.1381 \mathrm{E}-11 \quad 3.3628 \mathrm{E}-12$

$\begin{array}{lllllll}2.4000 \mathrm{E}+01 & 8.2729 \mathrm{E}-01 & 6.1976 \mathrm{E}-05 & 6.9058 \mathrm{E}-09 & 2.4244 \mathrm{E}-11 & 3.9577 \mathrm{E}-12\end{array}$

$\begin{array}{llllllll}2.5000 \mathrm{E}+01 & 8.2078 \mathrm{E}-01 & 6.4312 \mathrm{E}-05 & 7.4742 \mathrm{E}-09 & 2.7347 \mathrm{E}-11 & 4.6256 \mathrm{E}-12\end{array}$

$2.6000 \mathrm{E}+01 \quad 8.1432 \mathrm{E}-01 \quad 6.6629 \mathrm{E}-05 \quad 8.0635 \mathrm{E}-09 \quad 3.0699 \mathrm{E}-11 \quad 5.3719 \mathrm{E}-12$

$\begin{array}{lllllll}2.7000 \mathrm{E}+01 & 8.0791 \mathrm{E}-01 & 6.8928 \mathrm{E}-05 & 8.6736 \mathrm{E}-09 & 3.4310 \mathrm{E}-11 & 6.2019 \mathrm{E}-12\end{array}$

$\begin{array}{lllllll}2.8000 \mathrm{E}+01 & 8.0156 \mathrm{E}-01 & 7.1210 \mathrm{E}-05 & 9.3042 \mathrm{E}-09 & 3.8187 \mathrm{E}-11 & 7.1211 \mathrm{E}-12\end{array}$

$\begin{array}{llllll}2.9000 \mathrm{E}+01 & 7.9525 \mathrm{E}-01 & 7.3473 \mathrm{E}-05 & 9.9554 \mathrm{E}-09 & 4.2341 \mathrm{E}-11 & 8.1350 \mathrm{E}-12\end{array}$

$\begin{array}{llllll}3.0000 \mathrm{E}+01 & 7.8899 \mathrm{E}-01 & 7.5718 \mathrm{E}-05 & 1.0627 \mathrm{E}-08 & 4.6779 \mathrm{E}-11 & 9.2494 \mathrm{E}-12\end{array}$

$3.1000 \mathrm{E}+01 \quad 7.8278 \mathrm{E}-01 \quad 7.7946 \mathrm{E}-05 \quad 1.1318 \mathrm{E}-08 \quad 5.1511 \mathrm{E}-11 \quad 1.0470 \mathrm{E}-11$

$\begin{array}{lllllll}3.2000 E+01 & 7.7662 \mathrm{E}-01 & 8.0156 \mathrm{E}-05 & 1.2030 \mathrm{E}-08 & 5.6544 \mathrm{E}-11 & 1.1803 \mathrm{E}-11\end{array}$

$\begin{array}{lllllll}3.3000 \mathrm{E}+01 & 7.7051 \mathrm{E}-01 & 8.2349 \mathrm{E}-05 & 1.2761 \mathrm{E}-08 & 6.1888 \mathrm{E}-11 & 1.3253 \mathrm{E}-11\end{array}$

$\begin{array}{lllllll}3.4000 \mathrm{E}+01 & 7.6445 \mathrm{E}-01 & 8.4524 \mathrm{E}-05 & 1.3512 \mathrm{E}-08 & 6.7550 \mathrm{E}-11 & 1.4828 \mathrm{E}-11\end{array}$

$\begin{array}{lllllll}3.5000 \mathrm{E}+01 & 7.5843 \mathrm{E}-01 & 8.6683 \mathrm{E}-05 & 1.4283 \mathrm{E}-08 & 7.3539 \mathrm{E}-11 & 1.6533 \mathrm{E}-11\end{array}$

$\begin{array}{lllllll}3.6000 \mathrm{E}+01 & 7.5247 \mathrm{E}-01 & 8.8824 \mathrm{E}-05 & 1.5072 \mathrm{E}-08 & 7.9864 \mathrm{E}-11 & 1.8374 \mathrm{E}-11\end{array}$

$\begin{array}{lllllll}3.7000 \mathrm{E}+01 & 7.4654 \mathrm{E}-01 & 9.0949 \mathrm{E}-05 & 1.5882 \mathrm{E}-08 & 8.6532 \mathrm{E}-11 & 2.0358 \mathrm{E}-11\end{array}$

$\begin{array}{lllllll}3.8000 \mathrm{E}+01 & 7.4067 \mathrm{E}-01 & 9.3057 \mathrm{E}-05 & 1.6710 \mathrm{E}-08 & 9.3552 \mathrm{E}-11 & 2.2490 \mathrm{E}-11\end{array}$

$\begin{array}{llllll}3.9000 \mathrm{E}+01 & 7.3484 \mathrm{E}-01 & 9.5148 \mathrm{E}-05 & 1.7557 \mathrm{E}-08 & 1.0093 \mathrm{E}-10 & 2.4777 \mathrm{E}-11\end{array}$ $\begin{array}{llllll}4.0000 \mathrm{E}+01 & 7.2906 \mathrm{E}-01 & 9.7223 \mathrm{E}-05 & 1.8422 \mathrm{E}-08 & 1.0868 \mathrm{E}-10 & 2.7226 \mathrm{E}-11\end{array}$ $\begin{array}{llllll}4.1000 \mathrm{E}+01 & 7.2332 \mathrm{E}-01 & 9.9281 \mathrm{E}-05 & 1.9307 \mathrm{E}-08 & 1.1680 \mathrm{E}-10 & 2.9843 \mathrm{E}-11\end{array}$ $\begin{array}{llllll}4.2000 \mathrm{E}+01 & 7.1763 \mathrm{E}-01 & 1.0132 \mathrm{E}-04 & 2.0209 \mathrm{E}-08 & 1.2531 \mathrm{E}-10 & 3.2634 \mathrm{E}-11\end{array}$ $\begin{array}{lllllll}4.3000 \mathrm{E}+01 & 7.1198 \mathrm{E}-01 & 1.0335 \mathrm{E}-04 & 2.1130 \mathrm{E}-08 & 1.3421 \mathrm{E}-10 & 3.5606 \mathrm{E}-11\end{array}$ $\begin{array}{llllll}4.4000 \mathrm{E}+01 & 7.0638 \mathrm{E}-01 & 1.0536 \mathrm{E}-04 & 2.2070 \mathrm{E}-08 & 1.4350 \mathrm{E}-10 & 3.8766 \mathrm{E}-11\end{array}$ $\begin{array}{llllll}4.5000 \mathrm{E}+01 & 7.0082 \mathrm{E}-01 & 1.0735 \mathrm{E}-04 & 2.3027 \mathrm{E}-08 & 1.5321 \mathrm{E}-10 & 4.2120 \mathrm{E}-11\end{array}$ $\begin{array}{lllllll}4.6000 E+01 & 6.9531 E-01 & 1.0933 E-04 & 2.4002 E-08 & 1.6332 E-10 & 4.5674 E-11\end{array}$ $\begin{array}{lllllll}4.7000 \mathrm{E}+01 & 6.8984 \mathrm{E}-01 & 1.1130 \mathrm{E}-04 & 2.4995 \mathrm{E}-08 & 1.7386 \mathrm{E}-10 & 4.9436 \mathrm{E}-11\end{array}$ $\begin{array}{llllll}4.8000 \mathrm{E}+01 & 6.8441 \mathrm{E}-01 & 1.1324 \mathrm{E}-04 & 2.6005 \mathrm{E}-08 & 1.8483 \mathrm{E}-10 & 5.3411 \mathrm{E}-11\end{array}$ $\begin{array}{lllllll}4.9000 \mathrm{E}+01 & 6.7902 \mathrm{E}-01 & 1.1518 \mathrm{E}-04 & 2.7033 \mathrm{E}-08 & 1.9624 \mathrm{E}-10 & 5.7608 \mathrm{E}-11\end{array}$ $\begin{array}{lllllll}5.0000 \mathrm{E}+01 & 6.7368 \mathrm{E}-01 & 1.1709 \mathrm{E}-04 & 2.8078 \mathrm{E}-08 & 2.0809 \mathrm{E}-10 & 6.2031 \mathrm{E}-11\end{array}$

\section{$\underline{\text { Pu-241.Ist }}$}

\begin{tabular}{|c|c|c|}
\hline 5 & 1 & 'Ci' \\
\hline 'Pu-241' & 'Am-241' & 'Np-237' 'U-233' \\
\hline 14.399999 & 6185303 & 432.200012207031 \\
\hline 241 & 237 & 229 \\
\hline
\end{tabular}


Rev. 06

Title: $\quad$ Facility for Use in the Facility Performance Assessment

Baseline Radionuclide Inventory for the Remote-Handled Low-Level Waste Disposal

ECAR No.: $3940 \quad$ Rev. No.: $0 \quad$ Project No.: 31055

Date: $01 / 24 / 18$

\begin{tabular}{|c|c|c|c|c|c|}
\hline 7 & 7 & & \multicolumn{3}{|c|}{ ntimes } \\
\hline 8 & 31, & 1 & t1 t2 tp & & \\
\hline ty in & $\mathrm{Ci}$ & & & & \\
\hline Parameter & $\mathrm{Pu}-241$ & $A m-241$ & $\mathrm{~Np}-237$ & $U-233$ & Th-229 \\
\hline Lambda (1/y) & $4.814 \mathrm{E}-02$ & $1.604 \mathrm{E}-03$ & $3.239 E-07$ & $4.373 E-06$ & $9.443 E-05$ \\
\hline SpA Ci/g & $1.030 \mathrm{E}+02$ & $3.433 E+00$ & $7.050 \mathrm{E}-04$ & $9.682 \mathrm{E}-03$ & $2.127 \mathrm{E}-01$ \\
\hline Time (yr) & $\mathrm{Pu}-241$ & $\mathrm{Am}-241$ & $N p-237$ & $U-233$ & Th-229 \\
\hline
\end{tabular}

$\begin{array}{llllll}1.0000 \mathrm{E}+00 & 9.5300 \mathrm{E}-01 & 1.5645 \mathrm{E}-03 & 2.5548 \mathrm{E}-10 & 3.7395 \mathrm{E}-16 & 7.8046 \mathrm{E}-21\end{array}$

$2.0000 \mathrm{E}+00 \quad 9.0822 \mathrm{E}-01 \quad 3.0530 \mathrm{E}-03 \quad 1.0053 \mathrm{E}-09 \quad 2.9550 \mathrm{E}-15 \quad 1.4214 \mathrm{E}-19$

$\begin{array}{llllll}3.0000 \mathrm{E}+00 & 8.6554 \mathrm{E}-01 & 4.4690 \mathrm{E}-03 & 2.2254 \mathrm{E}-09 & 9.8520 \mathrm{E}-15 & 7.0294 \mathrm{E}-19\end{array}$

$\begin{array}{lllllll}4.0000 \mathrm{E}+00 & 8.2486 \mathrm{E}-01 & 5.8160 \mathrm{E}-03 & 3.8929 \mathrm{E}-09 & 2.3071 \mathrm{E}-14 & 2.2000 \mathrm{E}-18\end{array}$

$\begin{array}{lllllll}5.0000 \mathrm{E}+00 & 7.8610 \mathrm{E}-01 & 7.0972 \mathrm{E}-03 & 5.9859 \mathrm{E}-09 & 4.4520 \mathrm{E}-14 & 5.3190 \mathrm{E}-18\end{array}$

$\begin{array}{lllllll}6.0000 \mathrm{E}+00 & 7.4915 \mathrm{E}-01 & 8.3157 \mathrm{E}-03 & 8.4837 \mathrm{E}-09 & 7.6015 \mathrm{E}-14 & 1.0924 \mathrm{E}-17\end{array}$

$\begin{array}{llllll}7.0000 \mathrm{E}+00 & 7.1395 \mathrm{E}-01 & 9.4744 \mathrm{E}-03 & 1.1366 \mathrm{E}-08 & 1.1928 \mathrm{E}-13 & 2.0044 \mathrm{E}-17\end{array}$

$\begin{array}{lllllll}8.0000 \mathrm{E}+00 & 6.8039 \mathrm{E}-01 & 1.0576 \mathrm{E}-02 & 1.4615 \mathrm{E}-08 & 1.7596 \mathrm{E}-13 & 3.3871 \mathrm{E}-17\end{array}$

$\begin{array}{lllllll}9.0000 \mathrm{E}+00 & 6.4842 \mathrm{E}-01 & 1.1624 \mathrm{E}-02 & 1.8212 \mathrm{E}-08 & 2.4762 \mathrm{E}-13 & 5.3744 \mathrm{E}-17\end{array}$

$\begin{array}{llllll}1.0000 \mathrm{E}+01 & 6.1795 \mathrm{E}-01 & 1.2620 \mathrm{E}-02 & 2.2139 \mathrm{E}-08 & 3.3573 \mathrm{E}-13 & 8.1145 \mathrm{E}-17\end{array}$

$\begin{array}{llllll}1.1000 \mathrm{E}+01 & 5.8891 \mathrm{E}-01 & 1.3566 \mathrm{E}-02 & 2.6381 \mathrm{E}-08 & 4.4171 \mathrm{E}-13 & 1.1770 \mathrm{E}-16\end{array}$

$\begin{array}{llllll}1.2000 \mathrm{E}+01 & 5.6123 \mathrm{E}-01 & 1.4466 \mathrm{E}-02 & 3.0922 \mathrm{E}-08 & 5.6690 \mathrm{E}-13 & 1.6515 \mathrm{E}-16\end{array}$

$\begin{array}{lllllll}1.3000 \mathrm{E}+01 & 5.3486 \mathrm{E}-01 & 1.5321 \mathrm{E}-02 & 3.5747 \mathrm{E}-08 & 7.1258 \mathrm{E}-13 & 2.2538 \mathrm{E}-16\end{array}$

$\begin{array}{llllll}1.4000 \mathrm{E}+01 & 5.0972 \mathrm{E}-01 & 1.6133 \mathrm{E}-02 & 4.0842 \mathrm{E}-08 & 8.7995 \mathrm{E}-13 & 3.0038 \mathrm{E}-16\end{array}$

$1.5000 \mathrm{E}+01 \quad 4.8577 \mathrm{E}-01 \quad 1.6904 \mathrm{E}-02 \quad 4.6194 \mathrm{E}-08 \quad 1.0702 \mathrm{E}-12 \quad 3.9224 \mathrm{E}-16$

$1.6000 \mathrm{E}+01 \quad 4.6294 \mathrm{E}-01 \quad 1.7637 \mathrm{E}-02 \quad 5.1789 \mathrm{E}-08 \quad 1.2843 \mathrm{E}-12 \quad 5.0317 \mathrm{E}-16$

$1.7000 \mathrm{E}+01 \quad 4.4118 \mathrm{E}-01 \quad 1.8333 \mathrm{E}-02 \quad 5.7615 \mathrm{E}-08 \quad 1.5235 \mathrm{E}-12 \quad 6.3549 \mathrm{E}-16$

$1.8000 \mathrm{E}+01 \quad 4.2045 \mathrm{E}-01 \quad 1.8994 \mathrm{E}-02 \quad 6.3662 \mathrm{E}-08 \quad 1.7886 \mathrm{E}-12 \quad 7.9160 \mathrm{E}-16$

$\begin{array}{llllll}1.9000 \mathrm{E}+01 & 4.0069 \mathrm{E}-01 & 1.9622 \mathrm{E}-02 & 6.9916 \mathrm{E}-08 & 2.0805 \mathrm{E}-12 & 9.7399 \mathrm{E}-16\end{array}$

$\begin{array}{lllllll}2.0000 \mathrm{E}+01 & 3.8186 \mathrm{E}-01 & 2.0217 \mathrm{E}-02 & 7.6369 \mathrm{E}-08 & 2.4003 \mathrm{E}-12 & 1.1852 \mathrm{E}-15\end{array}$

$2.1000 \mathrm{E}+01 \quad 3.6391 \mathrm{E}-01 \quad 2.0782 \mathrm{E}-02 \quad 8.3010 \mathrm{E}-08 \quad 2.7487 \mathrm{E}-12 \quad 1.4280 \mathrm{E}-15$

$\begin{array}{lllllll}2.2000 \mathrm{E}+01 & 3.4681 \mathrm{E}-01 & 2.1318 \mathrm{E}-02 & 8.9828 \mathrm{E}-08 & 3.1266 \mathrm{E}-12 & 1.7050 \mathrm{E}-15\end{array}$

$\begin{array}{llllll}2.3000 \mathrm{E}+01 & 3.3051 \mathrm{E}-01 & 2.1827 \mathrm{E}-02 & 9.6816 \mathrm{E}-08 & 3.5346 \mathrm{E}-12 & 2.0192 \mathrm{E}-15\end{array}$

$\begin{array}{llllll}2.4000 \mathrm{E}+01 & 3.1498 \mathrm{E}-01 & 2.2309 \mathrm{E}-02 & 1.0396 \mathrm{E}-07 & 3.9736 \mathrm{E}-12 & 2.3732 \mathrm{E}-15\end{array}$

$\begin{array}{lllllll}2.5000 \mathrm{E}+01 & 3.0018 \mathrm{E}-01 & 2.2766 \mathrm{E}-02 & 1.1127 \mathrm{E}-07 & 4.4441 \mathrm{E}-12 & 2.7702 \mathrm{E}-15\end{array}$

$\begin{array}{llllll}2.6000 \mathrm{E}+01 & 2.8607 \mathrm{E}-01 & 2.3199 \mathrm{E}-02 & 1.1871 \mathrm{E}-07 & 4.9469 \mathrm{E}-12 & 3.2131 \mathrm{E}-15\end{array}$

$\begin{array}{llllll}2.7000 \mathrm{E}+01 & 2.7263 \mathrm{E}-01 & 2.3609 \mathrm{E}-02 & 1.2629 \mathrm{E}-07 & 5.4826 \mathrm{E}-12 & 3.7049 \mathrm{E}-15\end{array}$

$\begin{array}{lllllll}2.8000 \mathrm{E}+01 & 2.5981 \mathrm{E}-01 & 2.3998 \mathrm{E}-02 & 1.3400 \mathrm{E}-07 & 6.0516 \mathrm{E}-12 & 4.2489 \mathrm{E}-15\end{array}$

$\begin{array}{lllllll}2.9000 \mathrm{E}+01 & 2.4760 \mathrm{E}-01 & 2.4366 \mathrm{E}-02 & 1.4183 \mathrm{E}-07 & 6.6547 \mathrm{E}-12 & 4.8482 \mathrm{E}-15\end{array}$

$\begin{array}{llllll}3.0000 \mathrm{E}+01 & 2.3597 \mathrm{E}-01 & 2.4714 \mathrm{E}-02 & 1.4978 \mathrm{E}-07 & 7.2923 \mathrm{E}-12 & 5.5059 \mathrm{E}-15\end{array}$

$\begin{array}{llllll}3.1000 \mathrm{E}+01 & 2.2488 \mathrm{E}-01 & 2.5044 \mathrm{E}-02 & 1.5784 \mathrm{E}-07 & 7.9649 \mathrm{E}-12 & 6.2255 \mathrm{E}-15\end{array}$

$\begin{array}{llllll}3.2000 \mathrm{E}+01 & 2.1431 \mathrm{E}-01 & 2.5356 \mathrm{E}-02 & 1.6600 \mathrm{E}-07 & 8.6729 \mathrm{E}-12 & 7.0102 \mathrm{E}-15\end{array}$

$\begin{array}{lllllll}3.3000 \mathrm{E}+01 & 2.0424 \mathrm{E}-01 & 2.5650 \mathrm{E}-02 & 1.7427 \mathrm{E}-07 & 9.4169 \mathrm{E}-12 & 7.8634 \mathrm{E}-15\end{array}$

$\begin{array}{lllllll}3.4000 \mathrm{E}+01 & 1.9464 \mathrm{E}-01 & 2.5929 \mathrm{E}-02 & 1.8262 \mathrm{E}-07 & 1.0197 \mathrm{E}-11 & 8.7884 \mathrm{E}-15\end{array}$

$\begin{array}{llllll}3.5000 \mathrm{E}+01 & 1.8549 \mathrm{E}-01 & 2.6192 \mathrm{E}-02 & 1.9106 \mathrm{E}-07 & 1.1014 \mathrm{E}-11 & 9.7888 \mathrm{E}-15\end{array}$

$\begin{array}{lllllll}3.6000 \mathrm{E}+01 & 1.7678 \mathrm{E}-01 & 2.6440 \mathrm{E}-02 & 1.9958 \mathrm{E}-07 & 1.1868 \mathrm{E}-11 & 1.0868 \mathrm{E}-14\end{array}$

$\begin{array}{lllllll}3.7000 \mathrm{E}+01 & 1.6847 \mathrm{E}-01 & 2.6674 \mathrm{E}-02 & 2.0819 \mathrm{E}-07 & 1.2760 \mathrm{E}-11 & 1.2029 \mathrm{E}-14\end{array}$

$\begin{array}{llllllll}3.8000 \mathrm{E}+01 & 1.6055 \mathrm{E}-01 & 2.6895 \mathrm{E}-02 & 2.1686 \mathrm{E}-07 & 1.3689 \mathrm{E}-11 & 1.3277 \mathrm{E}-14\end{array}$

$\begin{array}{lllllll}3.9000 \mathrm{E}+01 & 1.5301 \mathrm{E}-01 & 2.7103 \mathrm{E}-02 & 2.2561 \mathrm{E}-07 & 1.4657 \mathrm{E}-11 & 1.4614 \mathrm{E}-14\end{array}$ $\begin{array}{lllllll}4.0000 \mathrm{E}+01 & 1.4582 \mathrm{E}-01 & 2.7299 \mathrm{E}-02 & 2.3442 \mathrm{E}-07 & 1.5662 \mathrm{E}-11 & 1.6043 \mathrm{E}-14\end{array}$ $\begin{array}{llllllll}4.1000 \mathrm{E}+01 & 1.3896 \mathrm{E}-01 & 2.7483 \mathrm{E}-02 & 2.4329 \mathrm{E}-07 & 1.6707 \mathrm{E}-11 & 1.7570 \mathrm{E}-14\end{array}$ $\begin{array}{lllllll}4.2000 \mathrm{E}+01 & 1.3243 \mathrm{E}-01 & 2.7657 \mathrm{E}-02 & 2.5222 \mathrm{E}-07 & 1.7790 \mathrm{E}-11 & 1.9197 \mathrm{E}-14\end{array}$ $\begin{array}{llllll}4.3000 \mathrm{E}+01 & 1.2621 \mathrm{E}-01 & 2.7820 \mathrm{E}-02 & 2.6121 \mathrm{E}-07 & 1.8913 \mathrm{E}-11 & 2.0927 \mathrm{E}-14\end{array}$ $\begin{array}{lllllll}4.4000 \mathrm{E}+01 & 1.2028 \mathrm{E}-01 & 2.7972 \mathrm{E}-02 & 2.7024 \mathrm{E}-07 & 2.0075 \mathrm{E}-11 & 2.2766 \mathrm{E}-14\end{array}$ $\begin{array}{llllll}4.5000 \mathrm{E}+01 & 1.1463 \mathrm{E}-01 & 2.8116 \mathrm{E}-02 & 2.7932 \mathrm{E}-07 & 2.1276 \mathrm{E}-11 & 2.4716 \mathrm{E}-14\end{array}$ $\begin{array}{lllllll}4.6000 \mathrm{E}+01 & 1.0924 \mathrm{E}-01 & 2.8250 \mathrm{E}-02 & 2.8845 \mathrm{E}-07 & 2.2518 \mathrm{E}-11 & 2.6781 \mathrm{E}-14\end{array}$ $\begin{array}{lllllll}4.7000 \mathrm{E}+01 & 1.0410 \mathrm{E}-01 & 2.8376 \mathrm{E}-02 & 2.9762 \mathrm{E}-07 & 2.3799 \mathrm{E}-11 & 2.8965 \mathrm{E}-14\end{array}$ $\begin{array}{lllllll}4.8000 \mathrm{E}+01 & 9.9213 \mathrm{E}-02 & 2.8493 \mathrm{E}-02 & 3.0683 \mathrm{E}-07 & 2.5121 \mathrm{E}-11 & 3.1272 \mathrm{E}-14\end{array}$ $\begin{array}{llllllll}4.9000 \mathrm{E}+01 & 9.4550 \mathrm{E}-02 & 2.8603 \mathrm{E}-02 & 3.1608 \mathrm{E}-07 & 2.6483 \mathrm{E}-11 & 3.3705 \mathrm{E}-14\end{array}$ $\begin{array}{lllllll}5.0000 \mathrm{E}+01 & 9.0107 \mathrm{E}-02 & 2.8705 \mathrm{E}-02 & 3.2536 \mathrm{E}-07 & 2.7885 \mathrm{E}-11 & 3.6268 \mathrm{E}-14\end{array}$ 
TEM-10200-1

$03 / 01 / 2012$

ENGINEERING CALCULATIONS AND ANALYSIS

Page B1 of B4

Rev. 06

Title: $\quad$ Facility for Use in the Facility Performance Assessment

$\begin{array}{lllll}\text { ECAR No.: } 3940 & \text { Rev. No.: } 0 & \text { Project No.: } 31055 \quad \text { Date: } 01 / 24 / 18\end{array}$

\section{Appendix B}

\section{Referenced Emails}

Jeff Frazier email to Brett Welty, September 20, 2017

From: Jeff Frazier (Contractor) <jeff.frazier@unnpp.gov>

Date: Wed, Sep 20, 2017 at 10:48 AM

Subject: Re: RH-LLW inventory questions and requests

To: "Welty, Brett D" < brett.welty@inl.gov>, "Sondrup, A Jeffrey" <a.sondrup@inl.gov>

Cc: "Nottestad, Stacy M" < stacy.nottestad@inl.gov>, Brady J Orchard <brady.orchard@inl.gov>

Brett/Jeff,

NRF agrees the original 51 year inventory estimate provided is still valid as there have been no significant changes. Also, as noted in question 2, it is appropriate to shift the inventory projections provided to the right 5 years $(2015=2020$, etc..). This will also better align with the startup date of our new large waste cask (Container 2$)$. If you have any more questions, let me know.

Thanks, Jeff

On Tue, Sep 19, 2017 at 4:35 PM, Welty, Brett D < brett.welty@inl.gov> wrote:

Jeff and Stacy,

This is high priority. Would it be possible for each of you to review the attached questions and provide answers via email to facilitate update of PA documents. My apologies for the short notice.

Jeff, the first questions pertain to NRF waste.

Stacy, the second and third sets pertain to ATR and MFC respectively. Can you address these?

Also would it be possible to have M. Hall do the decay requested under MFC?

Your prompt attention is much appreciated.

Brett 
Title: $\quad$ Facility for Use in the Facility Performance Assessment

$\begin{array}{lllll}\text { ECAR No.: } 3940 & \text { Rev. No.: } & 0 & \text { Project No.: } 31055 & \text { Date: } 01 / 24 / 18\end{array}$

Stacy Nottestad email to Brett Welty, September 27, 2017

From: Nottestad, Stacy $\mathbf{M}$ <stacy.nottestad@inl.gov>

Date: Wed, Sep 27, 2017 at 8:30 AM

Subject: Re: RH-LLW inventory questions and requests

To: "Welty, Brett D" < brett.welty@inl.gov>

Cc: "Schafer, Annette L" <annette.schafer@inl.gov>, A Jeffrey Sondrup < a.sondrup@inl.gov>, Brady J Orchard <brady.orchard@inl.gov>

\section{ATR QUESTIONS/REQUEST}

1) Are these inventories still valid? Does the generator feel a compelling need to update the inventory estimates in ECAR-854 or ECAR-851? It is assumed that the resins will be shipped to the RH-LLW facility in NUPAC containers according to ECAR-851. It is understood that a different container, not the assumed ES 3-60B Cask container, will be used for the metal waste. Specific casks will not drive a need to modify the ECAR, but a significant inventory modification would. Based on this, we do not believe it is necessary to modify ECAR-854.

The inventories are still valid, the process is the same and will continue to generate within the inventories identified in ECAR-854 and ECAR-851. The inventories are conservative and no significant modification is needed.

2) Is it appropriate to apply the estimated inventories for metals and resins for the period 2015-2064, currently documented in the ECARs, to the period 2020-2069? This would simply shift the inventory dates by 5 years but would keep the relative inventory the same.

Yes, the process of how resins are generated remains the same and inventory values are conservative. The inventory values will remain the same as identified in the ECAR's.

3) The PA will provide the performance for the first 20 years of disposals in addition to providing the performance for the entire 50 years of disposals. Please provide a recommendation for splitting the inventory into 1) the first 20 years of RH-LLW operation (2020-2039) and 2) the last 30 years of RH-LLW operation (2040-2069, or specifically split the inventory by modifying the ECARS. A recommendation with specific instructions could be made via email while an ECAR update would require more effort. The simple answer would be that for ECAR-851, the filter medium (and approved filters and end-boxes if loaded) would be $20 / 50 \times 220=88$ shipments/containers of the identified source term $(40 \%$ of total source term $)$. For ECAR-854 activated waste, since the number of shipments for the first 20 years encompasses the material currently in the canal, plus two CICs, and the last 30 years has $3 \mathrm{CICs}$, the source term and number of shipments/containers would be the same (50\% of the total source term) for the first 20 years and the last 30 years).

4) What is the recommendation for taking the time-line provided in ECARS 854 and 851 and projecting them to the 2020-2069 and 2020-2039 time line. This is partially addressed by question \#3, but in your response can you --- for the record

The 50 year timeline for operation of the facility is captured in ECAR-851 and ECAR-854. The ECARs do not specifically come out and say that the 50-years will start in 2016 , that time frame is listed only as an example. The averages used in the calculations for number of shipments and projected source terms apply regardless of when the first waste shipment is received. If the first 20 -year projections are a milestone that needs to be captured somewhere, we could use the process I described in the previous response to break out the first 20-years of operation, but unless you desire to use the ECARs to document this milestone, the information is already there and you can document the first 20 -years in whatever form your milestone requires 
TEM-10200-1

$03 / 01 / 2012$

ENGINEERING CALCULATIONS AND ANALYSIS

Page B3 of B4

Rev. 06

Title: $\quad$ Facility for Use in the Facility Performance Assessment

$\begin{array}{lllll}\text { ECAR No.: } 3940 & \text { Rev. No.: } 0 & \text { Project No.: } 31055 \quad \text { Date: } 01 / 24 / 18\end{array}$

Stacy Nottestad email to Jeff Sondrup, October 31, 2017

From: Nottestad, Stacy M <stacy.nottestad@inl.gov>

Date: Tue, Oct 31, 2017 at 11:53 AM

Subject: Re: Question

To: "Sondrup, A Jeffrey" < $\underline{\text { a.sondrup@inl.gov> }}$

This waste is from the 1994 and $2004 \mathrm{CICs}$, so I would go with 15 to 25 years.

On Tue, Oct 31, 2017 at 11:29 AM, Sondrup, A Jeffrey <a.sondrup@inl.gov> wrote:

Stacy,

I would like to make the following statement regarding the $1 \mathrm{CIC}$ equivalent of waste currently in the ATR canal that will be disposed of after operations commence.

The $1 \mathrm{CIC}$ equivalent inventory currently in the ATR canal was not corrected for decay. It is estimated this waste could be as old as 15 to 20 years old at the time the facility opens.

Is 15 to 20 years a good estimate?

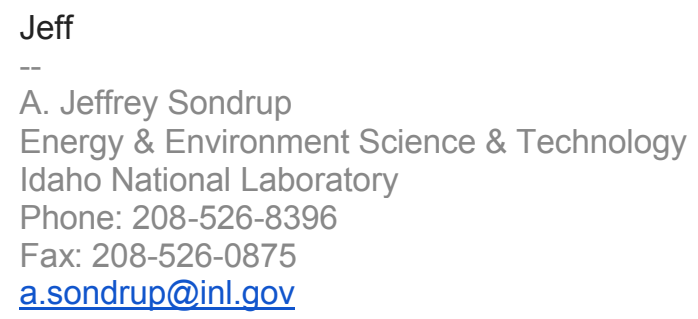

Jeff Frazier email to Jeff Sondrup, July 28, 2010

From: Frazier, Jeff W < Jeff.Frazier@unnpp.gov>

To: A Jeffrey Sondrup <A.Sondrup@inl.gov>

Date: Wed, Jul 28, 2010 at 10:48 AM

Subject: RE: Questions

Anita looked at our predominant structurals we will send long term and averaged their material content by weight and came up with an approximate material breakdown of $42 \%$ Zircaloy, $56 \%$ Nickel alloy (NiCrFe), and $2 \%$ Stanless Steel.

FYI, I'll be out next week if something comes up. Call Anita if needed.

Thanks

-----Original Message-----

From: A Jeffrey Sondrup [mailto:A.Sondrup@inl.gov]

Sent: Monday, July 26, 2010 5:20 PM

To: Frazier, Jeff W

Cc: David S Duncan

Subject: Questions

Jeff F., 
TEM-10200-1

$03 / 01 / 2012$

ENGINEERING CALCULATIONS AND ANALYSIS

Page B4 of B4

Rev. 06

Title: $\quad$ Facility for Use in the Facility Performance Assessment

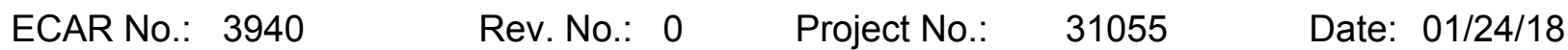

Can you tell me how much the waste containers for the metals (Type 3 ?) and resins (Type VI ?) weigh?

Also, in the first version of the inventory report (NRF-WS-473), it said the activated metal containers weighed about $2100 \mathrm{~kg}$ each. I assume this is activated metal and container weight. Can I assume the weight of activated metals is the difference between $2100 \mathrm{~kg}$ and the container weight?

NRF-WS-473 also said the resin containers were about $2000 \mathrm{~kg}$ each. Is this also the weight of resins and container?

Finally, can you tell me the types of activated metals in the waste and ballpark the weight percentage of each metal. For example, the ATR metals are $54 \%$ Aluminum, $46 \%$ SS, and $4 \%$ Inconel by weight.

As soon as you can. THANKS!

Jeff S. 